\title{
T-Spherical Fuzzy Rough Interactive Power Heronian Mean Aggregation Operators for Multiple Attribute Group Decision-Making
}

\author{
Haolun Wang ${ }^{1,2}$ (D) \\ 1 Research Center of the Central China for Economic and Social Development, Nanchang 330031, China; \\ haolunwang@ncu.edu.cn \\ 2 School of Economics and Management, Nanchang University, Nanchang 330031, China
}

Citation: Wang, H. T-Spherical Fuzzy Rough Interactive Power Heronian Mean Aggregation Operators for Multiple Attribute Group Decision-Making. Symmetry 2021, 13, 2422. https://doi.org/ $10.3390 /$ sym 13122422

Academic Editor:

José Carlos R. Alcantud

Received: 17 November 2021

Accepted: 9 December 2021

Published: 14 December 2021

Publisher's Note: MDPI stays neutral with regard to jurisdictional claims in published maps and institutional affiliations.

Copyright: (C) 2021 by the author. Licensee MDPI, Basel, Switzerland. This article is an open access article distributed under the terms and conditions of the Creative Commons Attribution (CC BY) license (https:// creativecommons.org/licenses/by/ $4.0 /)$.

\begin{abstract}
In this article, to synthesize the merits of interaction operational laws (IOLs), rough numbers (RNs), power average (PA) and Heronian mean (HM), a new notion of T-spherical fuzzy rough numbers (T-SFRNs) is first introduced to describe the intention of group experts accurately and take the interaction between individual experts into account with complete and symmetric information. The distance measure and ordering rules of T-SFRNs are proposed, and the IOLs of T-SFRNs are extended. Next, the PA and HM are combined based on the IOLs of T-SFRNs, and the T-Spherical fuzzy rough interaction power Heronian mean operator and its weighted form are proposed. These aggregation operators can accurately express both individual and group uncertainty using T-SFRNs, capture the interaction among membership degree, abstinence degree and nonmembership degree of T-SFRNs by employing IOLs, ensure the overall balance of variable values by the PA in the process of information fusion, and realize the interrelationship between attribute variables by the HM. Several properties and special cases of these aggregation operators are further presented and discussed. Subsequently, a new approach for dealing with T-spherical fuzzy multiple attribute group decision-making problems based on proposed aggregation operator is developed. Lastly, in order to validate the feasibility and reasonableness of the proposed approach, a numerical example is presented, and the superiorities of the proposed method are illustrated by describing a sensitivity analysis and a comparative analysis.
\end{abstract}

Keywords: multiple attribute group decision-making; T-spherical fuzzy rough numbers; Heronian mean operator; interaction operational laws; power average operator

\section{Introduction}

An attribute evaluation value usually embraces ambiguous and impermeable information in multiple attribute group decision-making (MAGDM) problems. However, as the actual group decision -making problem becomes more and more complex, scholars are faced with significant challenges in the expression of attribute variables. Fuzzy set (FS) [1] is an information representation method, which is widely used to solve information modeling problems with vague and uncertain information in many fields, but the FS has only one membership degree (MD) $\mathcal{M}(\hbar)(0 \leq \mathcal{M}(\hbar) \leq 1)$. This is not sufficient to comprehensively depict the uncertainty degree of humans' cognition of things. In view of this, Atanassov advanced intuitionistic fuzzy set (IFS) [2], which can describe more detailed information than the FS via the MD $\mathcal{M}(\hbar)$ and non-membership degree (NMD) $\mathcal{N}(\hbar)(0 \leq \mathcal{N}(\hbar) \leq 1)$. The characteristic of IFS is $\mathcal{M}(\hbar)+\mathcal{N}(\hbar) \leq 1$. In order to overcome the shortcoming that the IFS cannot be used in decision-making scenarios where the sum of $\mathcal{M}(\hbar)$ and $\mathcal{N}(\hbar)$ is greater than one, some scholars successively proposed Pythagorean fuzzy set (PyFS) $[3,4]$ $\left((\mathcal{M}(\hbar))^{2}+(\mathcal{N}(\hbar))^{2} \leq 1\right)$ and Fermatean fuzzy set (FFS) $[5,6]\left((\mathcal{M}(\hbar))^{3}+(\mathcal{N}(\hbar))^{3} \leq 1\right)$ with a wider range of decision-making information, and more generalized $q$-rung orthopair 
fuzzy $\left(q\right.$-ROF) $\left((\mathcal{M}(\hbar))^{q}+(\mathcal{N}(\hbar))^{q} \leq 1\right)$ [7]. Obviously, the IFS, PyFS and FFS are special cases of $q$-ROFS. The boundary of assessment information described by the MD and NMD also becomes larger when the parameter $q$ value increases in $q$-ROFS. Therefore, the comparison between $q$-ROFS and IFS, PyFS and FFS shows that the $q$-ROFS can provide more free space and flexibility for people to describe assessment information with preference. In recent years, the $q$-ROFS have been widely applied to the engineering and social fields [8-18].

The MD and NMD in $q$-ROFS, respectively, represent the parts belonging to and not belonging to a given set, while the rest is called uncertainty or hesitation degree, that is, the $q$-ROFS can only deal with the fuzzy concept of "neither this nor the other". However, in some decision-making situations (such as voting), people need to find more ways of responding with their opinions: Yes, Abstain, No, and Refuse [19]. Obviously, the $q$-ROFS is inadequate and unsuitable for expressing the opinions of decision-makers. Cuong first proposed an extended notion of picture fuzzy set (PFS) [19], and it can accurately express the evaluation opinions of decision-makers as an extension of IFS. The $\mathrm{MD}(0 \leq \mathcal{M}(\hbar) \leq 1)$ stands for "Yes", the abstinence degree (AD) $(0 \leq \Lambda(\hbar) \leq 1)$ expresses "Abstain", the NMD $(0 \leq \mathcal{N}(\hbar) \leq 1)$ indicates "No", and the rest of the whole set (i.e., Refuse degree (RD): $\pi(\hbar)=1-\mathcal{M}(\hbar)-\Lambda(\hbar)-\mathcal{N}(\hbar))$ means "Refuse" in the opinion. Furthermore, they must meet the restrictive condition that the sum is at most one. It is not difficult to discover that this set is capable of more accurately expressing the assessment information by extending the two-dimensional plane consisting of MD NMD to the three-dimensional space consisting of MD AD NMD. Therefore, the PFS is able to avoid the loss of evaluation information and improve the consistency between the acquired data and the actual decisionmaking environment [20]. In view of the fact that PFS cannot deal with the decision problem of $\mathcal{M}(\hbar)+\Lambda(\hbar)+\mathcal{N}(\hbar)>1$, the spherical fuzzy set (SFS) is an extended concept introduced by Ashraf and Abdullah in 2018 [21]. It features a larger decision space than the PFS; in other words, the quadratic sum of three functions cannot be larger than one. Inspired by the PyFS and $q$-ROFS, a more generalized T-spherical fuzzy set (T-SFS) $\left((\mathcal{M}(\hbar))^{t}+(\Lambda(\hbar))^{t}+(\mathcal{N}(\hbar))^{t} \leq 1(t \geq 1)\right)$ was advanced by Mahmood et al. [22]. It enables decision-makers to express "Yes", "Abstain", "No" and "Refuse" in their opinions with greater freedom and greater decision-making space. From the above, it is easy to find that the T-SFS has obvious generalizability, and this concept is related to the FS, IFS, PyFS, $q$-ROFS, PFS and SFS.

So far, the T-SFS has achieved abundant research results from theoretical study [22-24], information measurement [25-29] and practical application [22,30-33]. As an important part of T-SFS theory research, T-spherical fuzzy information aggregation technology has been studied by many scholars. Mahmood et al. [22] produced the weighted geometric aggregation operators (AO) for T-SFS. The T-spherical fuzzy generalized Maclarurin symmetric mean (MSM) (T-SFGMSM) operator and its weighted forms were introduced by Liu et al. [30]. On the basis of some Hamacher operations, Ullah et al. [31] proposed Hamacher AOs with T-spherical fuzzy numbers (T-SFNs). AOs with interval-valued TSFNs were developed by Ullah et al. [32]. Zeng SZ et al. [33] took the IOLs of T-SFNs into account and advanced some interactive average AOs with T-spherical fuzzy information. Grag et al. [34] introduced interactive geometric AOs with T-SFNs. Combining the superiority of the Muirhead mean (MM) and PA operators, power MM and power dual MM AOs were developed in T-spherical fuzzy environment by Liu et al. [35], as well as their weighted forms. Munir et al. [36] put forward some Einstein AOs of T-SFNs. Ju et al. [37] produced some AOs considering the interaction of functions in T-SFN and applied them to multi-attribute decision-making (MADM). Considering the advantages of T-SFS and PA operator, Garg et al. [38] defined several power AOs. Munir et al. [39] studied some associated immediate probability interactive geometric AOs. Liu et al. [40] developed the MSM AOs with normal T-spherical fuzzy information considering the interrelationship between multiple input variables. Mahmood et al. [41] advanced T-spherical fuzzy Dombi prioritized AOs, and combined with MULTIMOORA method to solve MADM problem. 


\subsection{Research Motivations}

Different AOs are expressed by different functions in the T-spherical fuzzy environment. Some AOs focus on eliminating the influence of absurd or biased data provided by people's preferences from the results, such as the PA operator [42]. Some AOs concern the correlation among input arguments, such as the MM [43] and MSM operators [44]. Other AOs emphasize the operation laws between T-SFNs, such as Algebraic operations [21], Hamacher operations [31], Einstein operations [36] and interactive operations [33,34,37]. At present, these studies only focus on the advantages of a certain dimension, and there are still insufficient studies on T-spherical fuzzy AOs that comprehensively consider the advantages of multi-dimensions. Therefore, until now, no AO has been proposed to fuse T-spherical fuzzy evaluation information based on the comprehensive consideration of the uncertainty of individual expert's viewpoint and expert groups' opinion, interactions between membership functions, the balance of input data and the interrelationship of attributes. To bridge this gap, the motivations of this paper are summarized as follows:

- In group decision-making process, each decision maker gives the initial T-SFNs for the assessment object and aggregates them according to certain rules. The relationship between T-SFNs given by individual decision-maker is ignored, which leads to the loss of part of the assessment information, so that the opinions of the decision-maker group cannot fully and accurately express. In other words, the T-SFNs given by individual decision-makers in group decision-making can only depict the ambiguity and hesitation of the individual evaluation of decision-makers (i.e., individual uncertainty), but cannot deal with the inaccuracy and subjectivity of group evaluation of decision-makers (i.e., group uncertainty). Therefore, the uncertainty in practical group decision-making problems cannot be fully expressed and dealt with only by T-SFN. In order to simultaneously and synthetically express the individual and group uncertainty of T-spherical fuzzy group decision-making problems, a new expression needs to be developed. The combination of fuzzy theory and rough set (RS) theory can form a more flexible and reliable expression through which to handle fuzzy assessment information from an overall perspective [45-47]; it can reflect the completeness and rationality of decision-makers' viewpoints. This paper observes that existing studies mainly focus on the relationship between the FSs or IFSs and rough sets [48,49], and seldom study the relationship between intuitionistic fuzzy numbers $[47,50,51]$. However, there is no research on T-SFNs.

- In terms of the IOLs of T-SFNs, the existing Algebraic, Einstein and Hamacher operations do not consider the interaction among the MD, AD and NMD in T-SFNs. For example, assuming that $\tilde{\wp}_{1}=<\mathcal{M}_{1}, \Lambda_{1}, \mathcal{N}_{1}>, \tilde{\wp}_{2}=<\mathcal{M}_{2}, \Lambda_{2}, \mathcal{N}_{2}>$ are two T-SFNs, if $\mathcal{N}_{1}=0, \Lambda_{2}=0$, and others are not 0 , then the result is that both AD and NMD are 0 based on T-SFNs algebraic sum operation [21,31], which is counterintuitive and needs to be overcome. Therefore, the use of the IOLs of T-SFNs for this purpose (IOLs-ZG) was introduced by Zeng SZ et al. [33] and Grag et al. [34]. Although the IOLs of T-SFNs can solve the above situation to a certain extent, they still feature limitations (see example 1). However, Ju et al. [37] proposed more generalized and universal IOLs of T-SFNs based on He et al. [52] (IOLs-J). IOLs- $J$ can deal with the above two defects.

- The decision-makers may give too high or too low abnormal preference values due to personal emotion or insufficient understanding of the decision object in the real world, which can make the decision-making process unfair. In order to eliminate this negative influence, this paper chooses the PA [42], which can mine the relative closeness of variables through the support degree, and then make the variables support and strengthen each other by assigning different power weights, to reflect the overall balance in the process of information fusion.

- In some decision situations, attribute variables are correlated with each other, which is objective and should not be ignored in the process of information aggregation. Existing AOs, such as the Bonferroni mean (BM) [53], HM [54,55], MM [43] and MSM [44] 
feature the ability to capture the interrelationship between attribute variables. MM and MSM operators have been extended in the T-spherical fuzzy environment $[30,35,40]$. Although MM and MSM operators feature more advantages than the HM and BM operators in this respect $[43,56]$, the calculation amount and computational complexity of MM and MSM operators are much higher than in HM and BM operators, especially when the number of attributes is large. With regard to HM and BM operators, Liu [57] indicated the former is more powerful than the latter, since the former is capable of assessing the interrelationship between an attribute variable and itself and reduce computational redundancy. In recent years, HM AOs have been successfully utilized in various kinds of fuzzy MAGDM [57-60], but there is no study on HM AOs with T-SFNs.

\subsection{Research Contributions}

In view of the above four motivations, the goal of this paper is to integrate the advantages of RNs, IOLs, PA and HM to solve practical decision-making problems. In this paper, there is a correlation between the concept of symmetry and the concepts of T-SFN and RN. The mixture of T-SFN and RN can be further investigated based on symmetry. A new concept of T-SFRN and its IOLs are introduced. The T-Spherical fuzzy rough interaction power Heronian mean (T-SFRIPHM) and its weighted AOs are developed. Furthermore, a MAGDM framework based on T-SFRIPWHM operator is established. In addition, the proposed framework is applied to an example to find the most appropriate alternative. The main contributions of this paper are described as follows.

- The concept of T-SFRN is proposed for the first time, and the distance measure, ordering rules and IOLs of T-SFRNs are extended.

- T-SFRIHM AOs are explored, along with their effective properties and special cases.

- The MAGDM framework is established based on the T-SFRIPWHM operator.

- Numerical examples are presented to illustrate the effectiveness of the MAGDM framework proposed in this paper. The advantages and scientificity of the proposed method are verified by sensitivity analysis and comparison with existing methods.

The other segments of this paper are structured as follows. Several basic notions about the T-SFS, HM and PA operators are introduced succinctly in Section 2. Some concepts of T-SFRNs are advanced in Section 3. The T-SFRIPHM and T-SFRIPWHM operators are developed in Section 4. A new approach for MAGDM problem based on the T-SFRIPWHM operator is advanced in Section 5. Subsequently, this method is applied to a given numerical example, and the sensitivity analysis and comparison analysis are reported in Section 6. Section 7 presents the concluding remarks.

\section{Preliminaries}

Here, some basic concepts of T-SFS, IOLs, PA and HM are introduced.

Definition 1 ([22]). Suppose $\mathcal{H}$ is a nonempty set, $\hbar \in \mathcal{H}$. A T-SFS $\widetilde{\Im}$ denoted on $\mathcal{H}$ is showed as follows:

$$
\tilde{\Im}=\left\{\left\langle\hbar,\left(\mathcal{M}_{\widetilde{\Im}}(\hbar), \Lambda_{\tilde{\Im}}(\hbar), \mathcal{N}_{\tilde{\Im}}(\hbar)\right)\right\rangle \mid \hbar \in \mathcal{H}\right\}
$$

where the functions $\mathcal{M}_{\widetilde{\Im}}(\hbar), \Lambda_{\widetilde{\Im}}(\hbar), \mathcal{N}_{\widetilde{\Im}}(\hbar)$ mean $M D, A D$ and $N M D$ of the $\hbar$ to $\widetilde{\Im}$, respectively. Satisfying $\mathcal{M}_{\widetilde{\Im}}(\hbar), \Lambda_{\widetilde{\Im}}(\hbar), \mathcal{N}_{\widetilde{\Im}}(\hbar) \in[0,1]$ and $\left(\mathcal{M}_{\widetilde{\Im}}(\hbar)\right)^{t}+\left(\Lambda_{\widetilde{\Im}}(\hbar)\right)^{t}+\left(\mathcal{N}_{\widetilde{\Im}}(\hbar)\right)^{t} \leq 1$ $(t \geq 1)$ for all $\hbar \in \mathcal{H}$. The RD of $\widetilde{\Im}$ is $\pi_{\widetilde{\Im}}(\hbar)=\sqrt[t]{1-\left(\mathcal{M}_{\tilde{\Im}}(\hbar)\right)^{t}-\left(\Lambda_{\widetilde{\Im}}(\hbar)\right)^{t}-\left(\mathcal{N}_{\widetilde{\Im}}(\hbar)\right)^{t}}$. For the convenience of information presentation, Mahmood et al. [32] and Liu et al. [40] called the triplet $\left\langle\mathcal{M}_{\widetilde{\Im}}(\hbar), \Lambda_{\widetilde{\Im}}(\hbar), \mathcal{N}_{\widetilde{\Im}}(\hbar)\right\rangle$ as $T-S F N$, which can be denoted by $\tilde{\wp}=\langle\mathcal{M}, \Lambda, \mathcal{N}\rangle$.

Remark 1. T-SFN is a generalization of all kinds of fuzzy numbers, its special cases are as follows: 
1. If $t=2$, the T-SFN reduces to the spherical fuzzy number (SFN) [22].

2. If $t=1$, the T-SFN reduces to the picture fuzzy number (PFN) [19].

3. If $\Lambda=0$, the T-SFN reduces to the q-rung orthopair fuzzy number (q-ROFN) [7].

4. If $t=2, \Lambda=0$, the T-SFN reduces to the Pythagorean fuzzy number (PyFN) [4].

5. If $t=1, \Lambda=0$, the T-SFN reduces to the intuitionistic fuzzy number (IFN) [2].

6. If $t=1, \Lambda=0, \mathcal{N}=0$, the T-SFN reduces to the classical fuzzy number (CFN) [1].

Definition 2 ([37]). Suppose $\tilde{\wp}=\langle\mathcal{M}, \Lambda, \mathcal{N}\rangle$ is a T-SFN, its score function sc is given as follows:

$$
\operatorname{sc}(\tilde{\wp})=\frac{1+\mathcal{M}^{q}-\Lambda^{q}-\mathcal{N}^{q}}{2}
$$

Its accuracy function ac is also given as follows:

$$
\operatorname{ac}(\tilde{\wp})=\mathcal{M}^{q}+\Lambda^{q}+\mathcal{N}^{q}
$$

Definition 3 ([37]). Suppose $\tilde{\wp}_{1}=<\mathcal{M}_{1}, \Lambda_{1}, \mathcal{N}_{1}>$ and $\tilde{\wp}_{2}=<\mathcal{M}_{2}, \Lambda_{2}, \mathcal{N}_{2}>$ are two T-SFNs. These T-SFNs can be ranked in the light of the following laws:

1. If $s c\left(\tilde{\wp}_{1}\right)>s c\left(\tilde{\wp}_{2}\right)$, then $\tilde{\wp}_{1} \succ \tilde{\wp}_{2}$;

2. If $s c\left(\tilde{\wp}_{1}\right)=s c\left(\tilde{\wp}_{2}\right)$, then: (i) If $a c\left(\tilde{\wp}_{1}\right)>a c\left(\tilde{\wp}_{2}\right)$, then $\tilde{\wp}_{1} \succ \tilde{\wp}_{2} ;$ (ii) If $\operatorname{ac}\left(\tilde{\wp}_{1}\right)=a c\left(\tilde{\wp}_{2}\right)$, then $\tilde{\wp}_{1}=\tilde{\wp}_{2}$.

Definition 4 ([22]). Suppose $\tilde{\wp}_{1}=<\mathcal{M}_{1}, \Lambda_{1}, \mathcal{N}_{1}>$ and $\tilde{\wp}_{2}=<\mathcal{M}_{2}, \Lambda_{2}, \mathcal{N}_{2}>$ are two T-SFNs, the T-SFNs algebraic operation laws are described as follows $(\lambda>0)$ :

1. $\tilde{\wp}_{1} \oplus \tilde{\wp}_{2}=\left\langle\sqrt[t]{\mathcal{M}_{1}^{t}+\mathcal{M}_{2}^{t}-\mathcal{M}_{1}^{t} \mathcal{M}_{2}^{t}}, \Lambda_{1} \Lambda_{2}, \mathcal{N}_{1} \mathcal{N}_{2}\right\rangle$

2. $\tilde{\wp}_{1} \otimes \tilde{\wp}_{2}=\left\langle\mathcal{M}_{1} \mathcal{M}_{2}, \sqrt[t]{\Lambda_{1}^{t}+\Lambda_{2}^{t}-\Lambda_{1}^{t} \Lambda_{2}^{t}}, \sqrt[t]{\mathcal{N}_{1}^{t}+\mathcal{N}_{2}^{t}-\mathcal{N}_{1}^{t} \mathcal{N}_{2}^{t}}\right\rangle$

3. $\lambda \tilde{\wp}_{1}=\left\langle\sqrt[t]{1-\left(1-\mathcal{M}_{1}^{t}\right)^{\lambda}}, \Lambda_{1}^{\lambda}, \mathcal{N}_{1}^{\lambda}\right\rangle$

4. $\quad \tilde{\wp}_{1}^{\lambda}=\left\langle\mathcal{M}_{1}^{\lambda}, \sqrt[t]{1-\left(1-\Lambda_{1}^{t}\right)^{\lambda}}, \sqrt[t]{1-\left(1-\mathcal{N}_{1}^{t}\right)^{\lambda}}\right\rangle$

He et al. [52] have proven that there are some shortcomings in the operation rules of algebraic sum and product in the intuitionistic fuzzy environment. For example, if the NMD of one intuitionistic fuzzy number (IFN) is 0 in the IFS, and the NMD of other IFNs is non-zero, then the final NMD is also 0 through the aggregation of algebraic sum operation rule, which is unreasonable and counterintuitive. Therefore, this situation exists not only in the algebraic operation rules of Definition 4, but also in the Einstein operation, Hamacher operation, and so on. In addition, there are some defects and limitations in the T-SFN IOLs defined by Zeng et al. [33] and Garg et al. [34] (IOLs-ZG), and the sum and product operations of any two T-SFNs may violate the constrain condition that the $t$ power sum of T-SFN's MD, AD and NMD is not greater than one.

Definition 5 ([34]). Let $\tilde{\wp}_{1}=<\mathcal{M}_{1}, \Lambda_{1}, \mathcal{N}_{1}>$ and $\tilde{\wp}_{2}=<\mathcal{M}_{2}, \Lambda_{2}, \mathcal{N}_{2}>$ be two T-SFNs, the interactive sum operation rule of T-SFNs is

$$
\tilde{\wp}_{1} \oplus \tilde{\wp}_{2}=\left\langle\sqrt[t]{1-\prod_{i=1}^{2}\left(1-\mathcal{M}_{i}^{t}\right)}, \sqrt[t]{1-\prod_{i=1}^{2}\left(1-\Lambda_{i}^{t}\right)}, \sqrt[t]{\prod_{i=1}^{2}\left(1-\mathcal{M}_{i}^{t}\right)-\prod_{i=1}^{2}\left(1-\mathcal{M}_{i}^{t}-\Lambda_{i}^{t}-\mathcal{N}_{i}^{t}\right)-\Lambda_{1}^{t} \Lambda_{2}^{t}}\right\rangle
$$


Example 1. Let $\tilde{\wp}_{1}=\langle 1,0,0\rangle, \tilde{\wp}_{2}=\langle 0,1,0\rangle$ be two T-SFNs, then obtain $\left.<1,1,0\right\rangle$ by using T-SFNs sum of IOLs, $(1)^{t}+(1)^{t}+(0)^{t}=2>1$; the result cannot satisfy the constrain condition of T-SFS: $\left(\mathcal{M}_{\tilde{\Im}}(\hbar)\right)^{t}+\left(\Lambda_{\tilde{\Im}}(\hbar)\right)^{t}+\left(\mathcal{N}_{\tilde{\Im}}(\hbar)\right)^{t} \leq 1(t \geq 1)$. Obviously, this is unreasonable.

To avoid the above unreasonable situation, Ju et al. [37] introduced the other IOLs of T-SFNs (IOLs-J) based on He et al. [52,61].

Definition 6 ([37]). Suppose $\tilde{\wp}_{1}=<\mathcal{M}_{1}, \Lambda_{1}, \mathcal{N}_{1}>$ and $\tilde{\wp}_{2}=<\mathcal{M}_{2}, \Lambda_{2}, \mathcal{N}_{2}>$ are two T-SFNs, the IOLs of T-SFNs are described as follows $(\lambda>0)$ :
1. $\quad \tilde{\wp}_{1} \oplus \tilde{\wp}_{2}=\left\langle\sqrt[t]{1-\prod_{i=1}^{2}\left(1-\mathcal{M}_{1}^{t}\right)}, \sqrt[t]{\prod_{i=1}^{2}\left(1-\mathcal{M}_{i}^{t}\right)-\prod_{i=1}^{2}\left(1-\mathcal{M}_{i}^{t}-\Lambda_{i}^{t}\right)}, \sqrt[t]{\prod_{i=1}^{2}\left(1-\mathcal{M}_{i}^{t}-\Lambda_{i}^{t}\right)-\prod_{i=1}^{2}\left(1-\mathcal{M}_{i}^{t}-\Lambda_{i}^{t}-\mathcal{N}_{1}^{t}\right)}\right\rangle$
2. $\tilde{\wp}_{1} \otimes \tilde{\wp}_{2}=\left\langle\sqrt[t]{\prod_{i=1}^{2}\left(1-\mathcal{N}_{i}^{t}-\Lambda_{i}^{t}\right)-\prod_{i=1}^{2}\left(1-\mathcal{N}_{i}^{t}-\Lambda_{i}^{t}-\mathcal{M}_{i}^{t}\right)}, \sqrt[t]{\prod_{i=1}^{2}\left(1-\mathcal{N}_{i}^{t}\right)-\prod_{i=1}^{2}\left(1-\mathcal{N}_{i}^{t}-\Lambda_{i}^{t}\right)}, \sqrt[t]{1-\prod_{i=1}^{2}\left(1-\mathcal{N}_{i}^{t}\right)}\right\rangle$
3. $\lambda \tilde{\wp}_{1}=\left\langle\sqrt[t]{1-\left(1-\mathcal{M}_{1}^{t}\right)^{\lambda}}, \sqrt[t]{\left(1-\mathcal{M}_{1}^{t}\right)^{\lambda}-\left(1-\mathcal{M}_{1}^{t}-\Lambda_{1}^{t}\right)^{\lambda}}, \sqrt[t]{\left(1-\mathcal{M}_{1}^{t}-\Lambda_{1}^{t}\right)^{\lambda}-\left(1-\mathcal{M}_{1}^{t}-\Lambda_{1}^{t}-\mathcal{N}_{1}^{t}\right)^{\lambda}}\right\rangle$
4. $\quad\left(\tilde{\wp}_{1}\right)^{\lambda}=\left\langle\sqrt[t]{\left(1-\mathcal{N}_{1}^{t}-\Lambda_{1}^{t}\right)^{\lambda}-\left(1-\mathcal{M}_{1}^{t}-\Lambda_{1}^{t}-\mathcal{N}_{1}^{t}\right)^{\lambda}}, \sqrt[t]{\left(1-\mathcal{N}_{1}^{t}\right)^{\lambda}-\left(1-\mathcal{N}_{1}^{t}-\Lambda_{1}^{t}\right)^{\lambda}}, \sqrt[t]{1-\left(1-\mathcal{N}_{1}^{t}\right)^{\lambda}}\right\rangle$

\section{Remark 2.}

1. If $t=2$, the IOLs of T-SFNs in Definition 6 reduce to the IOLs of SFNs.

2. If $t=1$, the IOLs of T-SFNs in Definition 6 reduce to the IOLs of PFNs [62].

3. If $t=0$, the IOLs of T-SFNs in Definition 6 reduce to the IOLs of $q$-ROFNs $[63,64]$.

4. If $t=2, \Lambda_{1}, \Lambda_{2}=0$, the IOLs of T-SFNs in Definition 6 reduce to the IOLs of PyFNs [65].

5. If $t=1, \Lambda_{1}, \Lambda_{2}=0$, the IOLs of T-SFNs in Definition 6 reduce to the IOLs of IFNs [52].

Theorem 1 ([37]). Supposing $\tilde{\wp}_{1}=<\mathcal{M}_{1}, \Lambda_{1}, \mathcal{N}_{1}>$ and $\tilde{\wp}_{2}=<\mathcal{M}_{2}, \Lambda_{2}, \mathcal{N}_{2}>$ are two T-SFNs, the operation properties are shown as follows $\left(\lambda_{1}, \lambda_{2}, \lambda>0\right)$ :
1. $\tilde{\wp}_{1} \oplus \tilde{\wp}_{2}=\tilde{\wp}_{2} \oplus \tilde{\wp}_{1}$
2. $\tilde{\wp}_{1} \otimes \tilde{\wp}_{2}=\tilde{\wp}_{2} \otimes \tilde{\wp}_{1}$
3. $\lambda\left(\tilde{\wp}_{1} \oplus \tilde{\wp}_{2}\right)=\lambda \tilde{\wp}_{1} \oplus \lambda \tilde{\wp}_{2}$
4. $\quad \lambda_{1} \tilde{\wp}_{1} \oplus \lambda_{2} \tilde{\wp}_{1}=\left(\lambda_{1}+\lambda_{2}\right) \tilde{\wp}_{1}$
5. $\left(\tilde{\wp}_{1}\right)^{\lambda_{1}} \otimes\left(\tilde{\wp}_{1}\right)^{\lambda_{2}}=\left(\tilde{\wp}_{1}\right)^{\lambda_{1}+\lambda_{2}}$
6. $\quad\left(\tilde{\wp}_{1}\right)^{\lambda} \otimes\left(\tilde{\wp}_{2}\right)^{\lambda}=\left(\tilde{\wp}_{1} \otimes \tilde{\wp}_{2}\right)^{\lambda}$

Definition 7 ([37]). Let $\tilde{\wp}_{\zeta}=\left\langle\mathcal{M}_{\zeta}, \Lambda_{\zeta}, \mathcal{N}_{\zeta}\right\rangle(\varsigma=1,2, \ldots, \kappa)$ be a set of T-SFNs, then T-SFWAI: $\Omega^{\kappa \rightarrow \Omega}$, if

$$
T-\operatorname{SFWAI}\left(\tilde{\wp}_{1}, \tilde{\wp}_{2}, \cdots, \tilde{\wp}_{\kappa}\right)=\bigoplus_{\zeta=1}^{\kappa} w_{\zeta} \tilde{\wp}_{\zeta}
$$

where $\Omega$ is a set of all T-SFNs, $\boldsymbol{w}=\left(w_{1}, w_{2}, \ldots, w_{\kappa}\right)^{T}$ is the weight vector of $\left(\tilde{\wp}_{1}, \tilde{\wp}_{2}, \ldots, \tilde{\wp}_{\kappa}\right)$, satisfying $w_{\varsigma} \in[0,1]$ and $\sum_{\varsigma=1}^{\kappa} w_{\varsigma}=1$.

Theorem 2 ([37]). Let $\tilde{\wp}_{i}=\left\langle\mathcal{M}_{i}, \Lambda_{i}, \mathcal{N}_{i}\right\rangle(i=1,2, \ldots, \kappa)$ be a collection of T-SFNs and $w=\left(w_{1}, w_{2}, \ldots, w_{\kappa}\right)^{T}$ be a weight vector of $\left(\tilde{\wp}_{1}, \tilde{\wp}_{2}, \cdots, \tilde{\wp}_{\kappa}\right)$, satisfying $w_{\varsigma} \in[0,1]$ and $\sum_{\zeta=1}^{\kappa} w_{\varsigma}=1$. The result of Equation (5) is still T-SFN, and

$$
\begin{aligned}
& T-\operatorname{SFWAI}\left(\tilde{\wp}_{1}, \tilde{\wp}_{2}, \cdots, \tilde{\wp}_{\mathcal{K}}\right)= \\
& \left\langle\begin{array}{l}
\sqrt[t]{1-\prod_{\zeta=1}^{\kappa}\left(1-\mathcal{M}_{\zeta}^{t}\right)^{w_{\zeta}}}, \sqrt[t]{\prod_{\zeta=1}^{\kappa}\left(1-\mathcal{M}_{\zeta}^{t}\right)^{w_{\zeta}}-\prod_{\zeta=1}^{\kappa}\left(1-\mathcal{M}_{\zeta}^{t}-\Lambda_{\zeta}^{t}\right)^{w_{\zeta}}} \\
\sqrt[t]{\prod_{\zeta=1}^{\kappa}\left(1-\mathcal{M}_{\zeta}^{t}-\Lambda_{\zeta}^{t}\right)^{w_{\zeta}}-\prod_{\zeta=1}^{\kappa}\left(1-\mathcal{M}_{\zeta}^{t}-\Lambda_{\zeta}^{t}-\mathcal{N}_{\zeta}^{t}\right)^{w_{\zeta}}}
\end{array}\right\rangle
\end{aligned}
$$

Definition 8 ([37]). Let $\tilde{\wp}_{1}=<\mathcal{M}_{1}, \Lambda_{1}, \mathcal{N}_{1}>$ and $\tilde{\wp}_{2}=<\mathcal{M}_{2}, \Lambda_{2}, \mathcal{N}_{2}>$ be two T-SFNs. The Hamming distance $(H D)$ between $\tilde{a}_{1}$ and $\tilde{a}_{2}$ is expressed as: 


$$
d_{H}\left(\tilde{\wp}_{1}, \tilde{\wp}_{2}\right)=\frac{\left|\mathcal{M}_{1}^{q}-\mathcal{M}_{2}^{q}\right|+\left|\Lambda_{1}^{q}-\Lambda_{2}^{q}\right|+\left|\mathcal{N}_{1}^{q}-\mathcal{N}_{2}^{q}\right|}{3}
$$

Definition 9 ([42]). Let $x_{\varsigma}(\varsigma=1,2, \ldots, \kappa)$ be any family of non-negative real number, if

$$
P A\left(x_{1}, x_{2}, \cdots, x_{\kappa}\right)=\sum_{\varsigma=1}^{\kappa} \frac{1+\mathcal{T}\left(x_{\varsigma}\right)}{\sum_{v=1}^{\kappa}\left(1+\mathcal{T}\left(x_{v}\right)\right)} x_{\varsigma}
$$

The PA is known as power average operator. $T\left(x_{\varsigma}\right)=\sum_{\zeta=1, \sigma \neq \varsigma}^{\mathcal{K}} \sup \left(x_{\zeta}, x_{\sigma}\right)(\zeta, \sigma=1,2, \ldots, \kappa)$, where $\sup \left(x_{\zeta}, x_{\sigma}\right)$ denotes the support degree between $x_{\zeta}$ and $x_{\sigma} . \sup \left(x_{\zeta}, x_{\sigma}\right)=1-d\left(x_{\zeta}, x_{\sigma}\right)$, where $d\left(x_{\zeta}, x_{\sigma}\right)$ means the distance between $x_{\varsigma}$ and $x_{\sigma}$. Furthermore, $\sup \left(x_{\zeta}, x_{\sigma}\right)$ meets the properties: (1) $\sup \left(x_{\varsigma}, x_{\sigma}\right) \in[0,1]$; (2) $\sup \left(x_{\varsigma}, x_{\sigma}\right)=\sup \left(x_{\sigma}, x_{\varsigma}\right)$; (3) if $d\left(x_{\varsigma}, x_{\sigma}\right) \leq d\left(x_{l}, x_{k}\right)$, the $\sup \left(x_{\zeta}, x_{\sigma}\right) \geq \sup \left(x_{l}, x_{k}\right)$.

Definition 10 ([55]). Let $\eta, \rho \geq 0, \eta$ and $\rho$ are not 0 at the same time, $x_{\varsigma}(\varsigma=1,2, \ldots, \kappa)$ be any family of non- negative real number, if

$$
H M^{\eta, \rho}\left(x_{1}, x_{2}, \cdots, x_{\kappa}\right)=\left(\frac{2}{\kappa(\kappa+1)} \sum_{\zeta=1, \xi=\varsigma}^{\kappa} x_{\zeta}^{\eta} x_{\xi}^{\rho}\right)^{\frac{1}{\eta+\rho}}
$$

The $H M^{\eta}, \rho$ is known as Heronian mean operator.

\section{T-SFRN}

\subsection{The Concept of the T-SFRN}

The T-SFS features a unique advantage in dealing with imprecise, vague, asymmetric and uncertain complex information, while the RS does not need any prior knowledge, and it can mine useful knowledge completely through the internal relationship of data. Therefore, the combination of T-SFS and RS can realize the complementary advantages of these two tools. This combination not only features data-driven characteristics and is not restricted by expert knowledge, but can also express the intention of individual and group decision-makers more comprehensively and objectively. Based on this, this subsection proposes a new concept of T-SFRN in the light of combining the concepts of T-SFN and RN.

Suppose that the finite non-empty set $Z$ is the universe, $\forall z \in Z$, the definition of T-SFRN assumes that $t$ class is equivalent to T-SFN class to form a family of T-spherical fuzzy topological sets, expressed as $R=\left\{\tilde{y}_{\zeta} \mid \varsigma=1,2, \ldots, \tau\right\}$. There is a certain dominant ordering relationship between each equivalent T-spherical fuzzy class, namely, $\tilde{y}_{1}<\tilde{y}_{2}<\ldots<\tilde{y}_{\varsigma}$, so for any class $\tilde{y}_{\varsigma} \in R, 1 \leq \varsigma \leq \tau$, $Y \subseteq Z, X \subseteq Z$. The upper approximation of $\tilde{y}_{\varsigma}$ can be defined as follows:

$$
\overline{\operatorname{Apr}}\left(\tilde{y}_{\varsigma}\right)=\cup\left\{Y \in Z / \Re(Y) \geq \tilde{y}_{\varsigma}, Y \subseteq X\right\}
$$

The lower approximation of $\tilde{y}_{\varsigma}$ can be defined as follows:

$$
\underline{\operatorname{Apr}}\left(\tilde{y}_{\varsigma}\right)=\cup\left\{Y \in Z / \Re(Y) \leq \tilde{y}_{\varsigma}, Y \cap X \neq \phi\right\}
$$

where $(Z, \Re)$ forms approximate $T$-spherical fuzzy rough space.

Based on the classical RN construction, any T-spherical fuzzy class $\tilde{y}_{\varsigma}$ can be expressed by T-SFRN, which consists of the T-spherical fuzzy rough lower limit (TSFRLL) $\underline{T-S F R N}\left(\tilde{y}_{\zeta}\right)$ and the T-spherical fuzzy rough upper limit (T-SFRUL) $\overline{T-S F R N}\left(\tilde{y}_{\varsigma}\right)$, and can be expressed as follows:

$$
\begin{aligned}
& \underline{T-\operatorname{SFRN}\left(\tilde{y}_{\zeta}\right)}=T-\operatorname{SFWAI}\left(\Re\left(Y_{1}\right), \Re\left(Y_{2}\right), \ldots, \Re\left(Y_{Q_{L}}\right)\right) \mid Y \in \underline{\operatorname{Apr}}\left(\tilde{y}_{\zeta}\right) \\
& \overline{T-\operatorname{SFRN}}\left(\tilde{y}_{\zeta}\right)=T-\operatorname{SFWAI}\left(\Re\left(Y_{1}\right), \Re\left(Y_{2}\right), \ldots, \Re\left(Y_{Q_{U}}\right)\right) \mid Y \in \overline{\operatorname{Apr}}\left(\tilde{y}_{\zeta}\right)
\end{aligned}
$$

T-SFRN $\left(\tilde{y}_{\zeta}\right)$ is obtained by utilizing the T-SFWAI operator [39] to aggregate T-SFN of $Y_{1}, Y_{2}, \ldots, Y_{Q_{L}}$ as $\Re\left(Y_{1}\right), \Re\left(Y_{2}\right), \ldots, \Re\left(Y_{Q_{L}}\right)$, and their weights are equal, namely, $w_{1}=w_{2}=\cdots=w_{Q_{L}}=1 / Q_{L}$. Similarly, $\overline{T-S F R N}\left(\tilde{y}_{\zeta}\right)$ can be obtained.

Definition 11. Based on the TSFRLL $\underline{T-S F R N}\left(\tilde{y}_{\zeta}\right)$ and TSFRUL $\overline{T-S F R N}\left(\tilde{y}_{\zeta}\right)$ of T-spherical fuzzy class $\tilde{y}_{\varsigma}, T$-SFRN $\left(\tilde{y}_{\zeta}\right)$ is defined as 


$$
T-\operatorname{SFRN}\left(\tilde{y}_{\varsigma}\right)=\left[\underline{T-\operatorname{SFRN}}\left(\tilde{y}_{\varsigma}\right), \overline{T-\operatorname{SFRN}}\left(\tilde{y}_{\varsigma}\right)\right]=\left[\left\langle\underline{\mathcal{M}_{\tilde{y}_{\varsigma}}}, \underline{\Lambda_{\tilde{y}_{\varsigma}}}, \underline{\mathcal{N}_{\tilde{y}_{\varsigma}}}\right\rangle,\left\langle\overline{\mathcal{M}_{\tilde{y}_{\varsigma}}}, \overline{\Lambda_{\tilde{y}_{\varsigma}}}, \overline{\mathcal{N}_{\tilde{y}_{\zeta}}}\right\rangle\right]
$$

For convenience of expression, Equation (14) can be expressed as

$$
\left[\tilde{y}_{\zeta}\right]=\left[\underline{\tilde{y}_{\zeta}}, \overline{\tilde{y}_{\zeta}}\right]=\left[\left\langle\left(\underline{\mathcal{M}_{\zeta}}, \underline{\Lambda_{\zeta}}, \underline{\mathcal{N}_{\zeta}}\right)\right\rangle,\left\langle\left(\overline{\mathcal{M}_{\zeta}}, \overline{\Lambda_{\zeta}}, \overline{\mathcal{N}_{\zeta}}\right)\right\rangle\right]
$$

Remark 3. The T-SFRN can be used as a generalized form of a variety of fuzzy rough framework. T-SFRN has the following special cases:

1. If $t=2$, the T-SFRN reduces to the spherical fuzzy rough number (SFRN).

2. If $t=1$, the T-SFRN reduces to the picture fuzzy rough number (PFRN).

3. If $\Lambda_{\zeta}, \overline{\Lambda_{\zeta}}=0$, the T-SFRN reduces to the q-rung orthopair fuzzy rough number ( $q$-ROFRN).

4. If $t=2, \Lambda_{\zeta}, \overline{\Lambda_{\zeta}}=0$, the T-SFRN reduces to the Pythagorean fuzzy rough number (PyFRN).

5. If $t=1, \overline{\Lambda_{S}}, \overline{\Lambda_{S}}=0$, the T-SFRN reduces to the intuitionistic fuzzy rough number (IFRN) [50,51].

6. If $t=1, \overline{\Lambda_{\zeta}}, \overline{\Lambda_{\zeta}}=0, \underline{\mathcal{N}_{\zeta}}, \overline{\mathcal{N}_{\zeta}}=0$, the T-SFRN reduces to the fuzzy rough number (FRN) $[66,67]$.

Theorem 3. For any T-spherical fuzzy class $\tilde{y}_{\varsigma}(\varsigma=1,2, \ldots, \tau)$, then $\underline{\tilde{y}_{\varsigma}} \leq \tilde{y}_{\varsigma} \leq \overline{\tilde{y}_{\varsigma}}$.

Proof. For each $\tilde{y}_{\zeta}(\zeta=1,2, \ldots, \tau)$, the $\operatorname{Apr}\left(\tilde{y}_{\zeta}\right)$ is composed of all T-spherical fuzzy classes that are not greater than $\tilde{y}_{\zeta}$, and the aggregation result through T-SFWAI operator is not greater than $\tilde{y}_{\zeta}$. Thus, $\underline{\tilde{y}_{\zeta}} \leq \tilde{y}_{\zeta}$. Similarly, $\tilde{y}_{\zeta} \leq \bar{y}_{\zeta}$ can be obtained. Therefore, Theorem 3 is true.

Theorem 4. Suppose $\left[\tilde{y}_{1}\right]$ and $\left[\tilde{y}_{2}\right]$ are two T-SFRNs, they have the following operation properties $(\lambda>0)$ :
1. $\left[\tilde{y}_{1}\right]+\left[\tilde{y}_{2}\right]=\left[\tilde{y}_{1} \oplus \tilde{y}_{2}, \tilde{y}_{1} \oplus \overline{\tilde{y}_{2}}\right]$
2. $\quad\left[\tilde{y}_{1}\right] \times\left[\tilde{y}_{2}\right]=\left[\underline{\tilde{y}_{1}} \otimes \underline{\tilde{y}_{2}}, \overline{\tilde{y}_{1}} \otimes \overline{\tilde{y}_{2}}\right]$
3. $\lambda\left[\tilde{y}_{1}\right]=\left[\lambda \tilde{y}_{1}, \overline{\lambda \tilde{y}_{1}}\right]$
4. $\quad\left[\tilde{y}_{1}\right]^{\lambda}=\left[\underline{\tilde{y}_{1}}, \overline{\tilde{y}_{1}} \lambda\right]$

Proof. Since T-SFRN $\left(\tilde{y}_{\zeta}\right)(1 \leq \varsigma \leq \tau)$ appears in the form of an interval, its operation property is the same as the general interval number, so it is easy to prove Theorem 4 .

Example 2. Suppose that four experts to evaluate an attribute, and the variables are expressed by T-SFN: $\tilde{y}_{1}=\langle 0.700,0.000,0.400\rangle, \tilde{y}_{2}=\langle 0.200,0.600,0.500\rangle, \tilde{y}_{3}=\langle 0.800,0.100,0.200\rangle$, $\tilde{y}_{4}=<0.600,0.500,0.000>$. According to Definition 2 and Definition $3(t=2)$, these T-SFNs are sorted as: $\tilde{y}_{2}<\tilde{y}_{4}<\tilde{y}_{1}<\tilde{y}_{3}$.

Taking $\tilde{y}_{1}$ as an example, it can be seen from the T-SFRN structure that there are $\underline{\operatorname{Apr}}\left(\tilde{y}_{1}\right)=\left\{\tilde{y}_{2}, \tilde{y}_{4}, \tilde{y}_{1}\right\}$ and $\overline{A p r}\left(\tilde{y}_{1}\right)=\left\{\tilde{y}_{1}, \tilde{y}_{3}\right\}$, then it can be obtained by Equations (12) and (13):

$\underline{T-\operatorname{SFRN}}\left(\tilde{y}_{1}\right)=T-\operatorname{SFWAI}\left(\tilde{y}_{1}, \tilde{y}_{2}, \tilde{y}_{4}\right)$

$=\left\langle\sqrt{1-\left(1-0.7^{2}\right)^{\frac{1}{3}} \times\left(1-0.2^{2}\right)^{\frac{1}{3}} \times\left(1-0.6^{2}\right)^{\frac{1}{3}}}, \sqrt{\begin{array}{c}\left(1-0.7^{2}\right)^{\frac{1}{3}} \times\left(1-0.2^{2}\right)^{\frac{1}{3}} \times\left(1-0.6^{2}\right)^{\frac{1}{3}}-\left(1-0.7^{2}-0^{2}\right)^{\frac{1}{3}} \\ \times\left(1-0.2^{2}-0.6^{2}\right)^{\frac{1}{3}} \times\left(1-0.6^{2}-0.5^{2}\right)^{\frac{1}{3}}\end{array}}\right.$

$\sqrt{\begin{array}{c}\left(1-0.7^{2}-0^{2}\right)^{\frac{1}{3}} \times\left(1-0.2^{2}-0.6^{2}\right)^{\frac{1}{3}} \times\left(1-0.6^{2}-0.5^{2}\right)^{\frac{1}{3}}-\left(1-0.7^{2}-0^{2}-0.4^{2}\right)^{\frac{1}{3}} \\ \times\left(1-0.2^{2}-0.6^{2}-0.5^{2}\right)^{\frac{1}{3}} \times\left(1-0.6^{2}-0.5^{2}-0.0^{2}\right)^{\frac{1}{3}}\end{array}}$

$=<0.566,0.432,0.360>$ 


$$
\begin{aligned}
& \overline{T-S F R N}\left(\tilde{y}_{1}\right)=T-\operatorname{SFWAI}\left(\tilde{y}_{1}, \tilde{y}_{3}\right) \\
& =\left\langle\sqrt{1-\left(1-0.7^{2}\right)^{\frac{1}{2}} \times\left(1-0.8^{2}\right)^{\frac{1}{2}}}, \sqrt{\begin{array}{c}
\left(1-0.7^{2}\right)^{\frac{1}{2}} \times\left(1-0.8^{2}\right)^{\frac{1}{2}}- \\
\left(1-0.7^{2}-0^{2}\right)^{\frac{1}{2}} \times\left(1-0.8^{2}-0.1^{2}\right)^{\frac{1}{2}}
\end{array}},\right.
\end{aligned}
$$

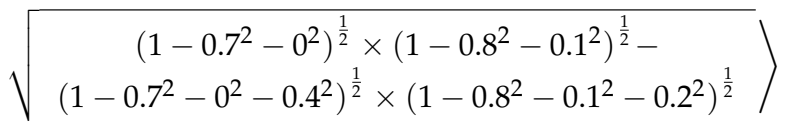

$$
\begin{aligned}
& =<0.756,0.077,0.305>
\end{aligned}
$$

Next, $\left[\tilde{y}_{1}\right]=[<0.566,0.432,0.360>,<0.756,0.077,0.305>]$.

Similarly, $\left.\left.\left[\tilde{y}_{2}\right]=[<0.200,0.600,0.500\rangle,<0.648,0.357,0.321\right\rangle\right] ;\left[\tilde{y}_{3}\right]=[<0.648,0.357,0.321\rangle$, $<0.800,0.100,0.200>] ;\left[\tilde{y}_{4}\right]=[<0.465,0.548,0.338>,<0.714,0.280,0.251>]$.

The significance of the T-SFNs' conversion into the T-SFRNs is that the initial T-SFN is only given by an individual expert, which ignores the interaction between experts and cannot accurately express the group opinions of experts. However, the T-SFRN is derived from a holistic perspective and can reflect the integrity and rationality of the experts' opinions. For example, the first expert's evaluation of the attribute is $\tilde{y}_{1}=\langle 0.700,0.000,0.400\rangle$, but from overall perspective, the attribute value should be $\left.\left.\left[\tilde{y}_{1}\right]=[<0.566,0.432,0.360\rangle,<0.756,0.077,0.305\right\rangle\right]$, which means the accurate value should be between the TSFRLL $<0.566,0.432,0.360>$ and the TSFRUL $<0.756,0.077,0.305>$. Thus, the T-SFRN should be all T-SFNs between the lower limit and the upper limit. In addition, the T-SFNs are transformed into T-SFRNs by applying the T-SFWAI operator, where taking IOLs of T-SFNs into account, so that the T-SFRN is more reasonable, and it can avoid the counterintuitive dilemmas caused by such as AD 0 in $\tilde{y}_{1}$ and NMD 0 in $\tilde{y}_{4}$. Therefore, the T-SFRNs can not only reflect the uncertainty of individuals and groups, but also avoid the loss or attenuation of information.

\subsection{The Compare Rules of T-SFNs}

In this subsection, the HD between two T-SFRNs is proposed based on the distance measure of T-SFNs.

Definition 12. Let $\left[\tilde{y}_{\varsigma}\right](\varsigma=1,2)$ be two any T-SFRNs, then the HD between $\left[\tilde{y}_{1}\right]$ and $\left[\tilde{y}_{2}\right]$ is defined as

$$
d_{H}\left(\left[\tilde{y}_{1}\right],\left[\tilde{y}_{2}\right]\right)=\frac{1}{6}\left(\left|\underline{\mathcal{M}}_{1}^{t}-\underline{\mathcal{M}}_{2}^{t}\right|+\left|\underline{\Lambda}_{1}^{t}-\underline{\Lambda}_{2}^{t}\right|+\left|\underline{\mathcal{N}}_{1}^{t}-\underline{\mathcal{N}}_{2}^{t}\right|+\left|\overline{\mathcal{M}}_{1}^{t}-\overline{\mathcal{M}}_{2}^{t}\right|+\left|\bar{\Lambda}_{1}^{t}-\bar{\Lambda}_{2}^{t}\right|+\left|\overline{\mathcal{N}}_{1}^{t}-\overline{\mathcal{N}}_{2}^{t}\right|\right)
$$

The HD between $\left[\tilde{y}_{1}\right]$ and $\left[\tilde{y}_{2}\right]$ features the following properties:

1. $0 \leq d_{H}\left(\left[\tilde{y}_{1}\right],\left[\tilde{y}_{2}\right]\right) \leq 1$,

2. $d_{H}\left(\left[\tilde{y}_{1}\right],\left[\tilde{y}_{2}\right]\right)=d_{H}\left(\left[\tilde{y}_{2}\right],\left[\tilde{y}_{1}\right]\right)$,

3. $d_{H}\left(\left[\tilde{y}_{1}\right],\left[\tilde{y}_{2}\right]\right)=0$, iff $\left[\tilde{y}_{1}\right]=\left[\tilde{y}_{2}\right]$.

To compare any two T-SFRNs, the score function $s c\left(\left[\tilde{y}_{\zeta}\right]\right)$ and the distance function $\operatorname{Dis}\left(\left[\tilde{y}_{\varsigma}\right]\right)$ of T-SFRN $\left[\tilde{y}_{\varsigma}\right]$ are advanced based on the TSFRLL and TSFRUL of the T-SFRN.

Considering that the TSFRLL and TSFRUL are T-SFNs, they are fused by the interaction sum operation, and the score function of one T-SFRN is obtained by utilizing the Equation (2) It is defined as:

Definition 13. Supposing $\left[\tilde{y}_{\varsigma}\right]$ is any T-SFRN, its score function $\operatorname{sc}\left(\left[\tilde{y}_{\varsigma}\right]\right)$ is

$$
s c\left(\left[\tilde{y}_{\zeta}\right]\right)=\frac{1}{2}\left(2-2\left(1-\underline{\mathcal{M}}_{\zeta}^{t}\right)\left(1-\overline{\mathcal{M}}_{\zeta}^{t}\right)+\left(1-\underline{\mathcal{M}}_{\zeta}^{t}-\underline{\Lambda}_{\zeta}^{t}-\underline{\mathcal{N}}_{\zeta}^{t}\right)\left(1-\overline{\mathcal{M}}_{\zeta}^{t}-\bar{\Lambda}_{\zeta}^{t}-\overline{\mathcal{N}}_{\zeta}^{t}\right)\right)
$$

According to Definition 8, the distance between the TSFRLL and TSFRUL of any T-SFRN is taken as the distance function of T-SFRN $\left[\tilde{y}_{c}\right]$, which is defined as:

Definition 14. Supposing $\left[\tilde{y}_{\varsigma}\right]$ is any T-SFRN, its distance function Dis $\left(\left[\tilde{y}_{\varsigma}\right]\right)$ is

$$
\operatorname{Dis}\left(\left[\tilde{y}_{\zeta}\right]\right)=d\left(\left[\left\langle\underline{\mathcal{M}}_{\zeta}, \underline{\Lambda}_{\zeta}, \underline{\mathcal{N}}_{\zeta}\right\rangle,\left\langle\overline{\mathcal{M}}_{\zeta}, \bar{\Lambda}_{\zeta}, \overline{\mathcal{N}}_{\zeta}\right\rangle\right]\right)=\frac{\left|\underline{\mathcal{M}}_{\zeta}^{t}-\overline{\mathcal{M}}_{\zeta}^{t}\right|+\left|\underline{\Lambda}_{\zeta}^{t}-\bar{\Lambda}_{\zeta}^{t}\right|+\left|\underline{\mathcal{N}}_{\zeta}^{t}-\overline{\mathcal{N}}_{\zeta}^{t}\right|}{3}
$$


Definition 15. The score function $\operatorname{sc}\left(\left[\tilde{y}_{\zeta}\right]\right)$ considers the interaction among the $M D, A D$ and $N M D$ in $\left[\tilde{y}_{\zeta}\right]$, and the distance function Dis( $\left.\left[\tilde{y}_{\varsigma}\right]\right)$ is the HD between the TSFRLL and TSFRUL of $\left[\tilde{y}_{\zeta}\right]$. Let $\left[\tilde{y}_{\varsigma}\right](\varsigma=1,2)$ be any two T-SFRNs, then the comparison rules of $\left[\tilde{y}_{\zeta}\right](\varsigma=1,2)$ are defined as:

1. If $s c\left(\left[\tilde{y}_{1}\right]\right)>s c\left(\left[\tilde{y}_{2}\right]\right)$, then $\left[\tilde{y}_{1}\right]>\left[\tilde{y}_{2}\right]$,

2. If $s c\left(\left[\tilde{y}_{1}\right]\right)=s c\left(\left[\tilde{y}_{2}\right]\right), \operatorname{Dis}\left(\left[\tilde{y}_{1}\right]\right)=\operatorname{Dis}\left(\left[\tilde{y}_{2}\right]\right)$, then $\left[\tilde{y}_{1}\right]=\left[\tilde{y}_{2}\right]$,

3. If $s c\left(\left[\tilde{y}_{1}\right]\right)=s c\left(\left[\tilde{y}_{2}\right]\right), \operatorname{Dis}\left(\left[\tilde{y}_{1}\right]\right)<\operatorname{Dis}\left(\left[\tilde{y}_{2}\right]\right)$, then $\left[\tilde{y}_{1}\right]>\left[\tilde{y}_{2}\right]$.

\subsection{The IoLs of T-SFRNs}

The T-spherical fuzzy rough interaction operation can be extended on the basis of Definition 6 .

Definition 16. Let $\left[\tilde{y}_{\varsigma}\right](\varsigma=1,2)$ be any two T-SFRNs. The IOLs of T-SFRNs are defined as $(\lambda>0)$ :

1. $\quad\left[\tilde{y}_{1}\right] \oplus\left[\tilde{y}_{2}\right]=\left[\begin{array}{l}\left.\left\langle\sqrt[t]{1-\prod_{\zeta=1}^{2}\left(1-\underline{\mathcal{M}_{\zeta}^{t}}\right)}, \sqrt[t]{\prod_{\zeta=1}^{2}\left(1-\underline{\mathcal{M}_{\zeta}^{t}}\right)-\prod_{\zeta=1}^{2}\left(1-\mathcal{M}_{\zeta}^{t}-\Lambda_{\zeta}^{t}\right.}\right), \sqrt[t]{\prod_{\zeta=1}^{2}\left(1-\mathcal{M}_{\zeta}^{t}-\underline{\Lambda}_{\zeta}^{t}\right)-\prod_{\zeta=1}^{2}\left(1-\underline{\mathcal{M}}_{\zeta}^{t}-\underline{\Lambda}_{\zeta}^{t}-\underline{\mathcal{N}}_{\zeta}^{t}\right)}\right\rangle, \\ \left\langle\sqrt[t]{1-\prod_{\zeta=1}^{2}\left(1-\overline{\mathcal{M}}_{\zeta}^{t}\right)}, \sqrt[t]{\prod_{\zeta=1}^{2}\left(1-\overline{\mathcal{M}}_{\zeta}^{t}\right)-\prod_{\zeta=1}^{2}\left(1-\overline{\mathcal{M}}_{\zeta}^{t}-\bar{\Lambda}_{\zeta}^{t}\right)}, \sqrt[t]{\prod_{\zeta=1}^{2}\left(1-\overline{\mathcal{M}}_{\zeta}^{t}-\bar{\Lambda}_{\zeta}^{t}\right)-\prod_{\zeta=1}^{2}\left(1-\overline{\mathcal{M}}_{\zeta}^{t}-\bar{\Lambda}_{\zeta}^{t}-\overline{\mathcal{N}}_{\zeta}^{t}\right)}\right\rangle\end{array}\right]$

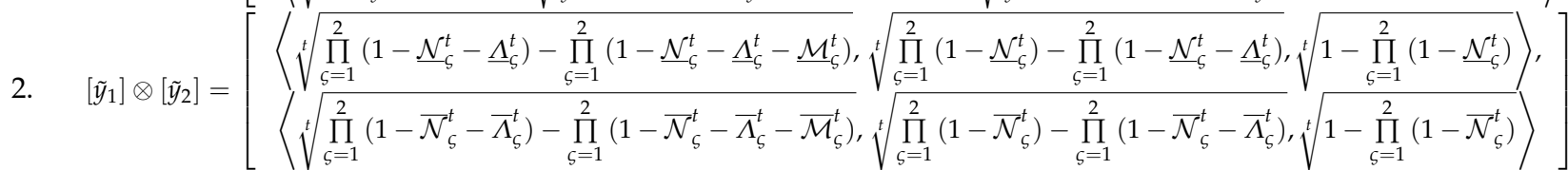

3. $\lambda\left[\tilde{y}_{1}\right]=\left[\begin{array}{l}\left\langle\sqrt[t]{1-\left(1-\underline{\mathcal{M}_{1}^{t}}\right)^{\lambda}}, \sqrt[t]{\left(1-\mathcal{M}_{1}^{t}\right)^{\lambda}-\left(1-\underline{\mathcal{M}}_{1}^{t}-\underline{\Lambda}_{1}^{t}\right)^{\lambda}}, \sqrt[t]{\left(1-\mathcal{M}_{1}^{t}-\Lambda_{1}^{t}\right)^{\lambda}-\left(1-\underline{\mathcal{M}}_{1}^{t}-\Lambda_{1}^{t}-\underline{\mathcal{N}}_{1}^{t}\right)^{\lambda}}\right\rangle, \\ \left\langle\sqrt[t]{1-\left(1-\overline{\mathcal{M}}_{1}^{t}\right)^{\lambda}}, \sqrt[t]{\left(1-\overline{\mathcal{M}}_{1}^{t}\right)^{\lambda}-\left(1-\overline{\mathcal{M}}_{1}^{t}-\bar{\Lambda}_{1}^{t}\right)^{\lambda}}, \sqrt[t]{\left(1-\overline{\mathcal{M}}_{1}^{t}-\bar{\Lambda}_{1}^{t}\right)^{\lambda}-\left(1-\overline{\mathcal{M}}_{1}^{t}-\bar{\Lambda}_{1}^{t}-\overline{\mathcal{N}}_{1}^{t}\right)^{\lambda}}\right\rangle\end{array}\right]$

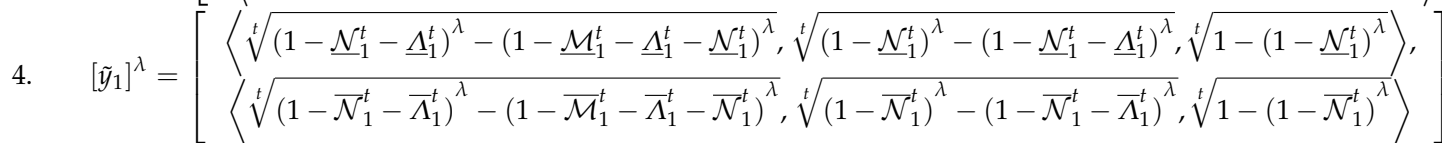

Theorem 5. Let $\left[\tilde{y}_{c}\right](\varsigma=1,2)$ be any two T-SFRNs. They have the following operation properties:

1. $\left[\tilde{y}_{1}\right] \oplus\left[\tilde{y}_{2}\right]=\left[\tilde{y}_{2}\right] \oplus\left[\tilde{y}_{1}\right]$

2. $\left[\tilde{y}_{1}\right] \otimes\left[\tilde{y}_{2}\right]=\left[\tilde{y}_{2}\right] \otimes\left[\tilde{y}_{1}\right]$

3. $\lambda\left(\left[\tilde{y}_{1}\right] \oplus\left[\tilde{y}_{2}\right]\right)=\lambda\left[\tilde{y}_{1}\right] \oplus \lambda\left[\tilde{y}_{2}\right](\lambda>0)$;

4. $\quad \lambda_{1}\left[\tilde{y}_{1}\right] \oplus \lambda_{2}\left[\tilde{y}_{1}\right]=\left(\lambda_{1}+\lambda_{2}\right)\left[\tilde{y}_{1}\right]\left(\lambda_{1}, \lambda_{2}>0\right)$;

5. $\quad\left[\tilde{y}_{1}\right]^{\lambda 1} \otimes\left[\tilde{y}_{1}\right]^{\lambda 2}=\left[\tilde{y}_{1}\right]^{\lambda 1+\lambda 2}\left(\lambda_{1}, \lambda_{2}>0\right)$;

6. $\left[\tilde{y}_{1}\right]^{\lambda} \otimes\left[\tilde{y}_{2}\right]^{\lambda}=\left(\left[\tilde{y}_{1}\right] \otimes\left[\tilde{y}_{2}\right]\right)^{\lambda}(\lambda>0)$.

Proof. According to the operational properties in the interval numbers, Properties 1 and 2 are easy to be proved. Property 3 is proven below:

According to Definition 16,

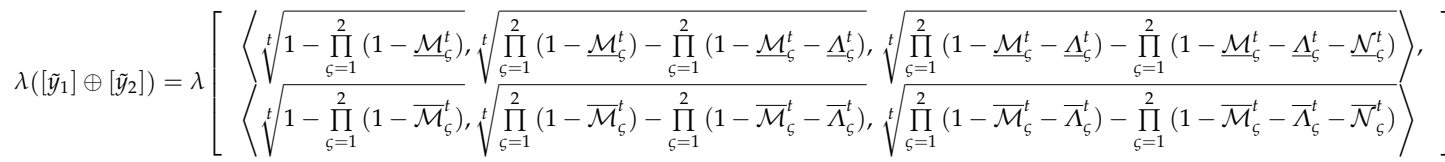

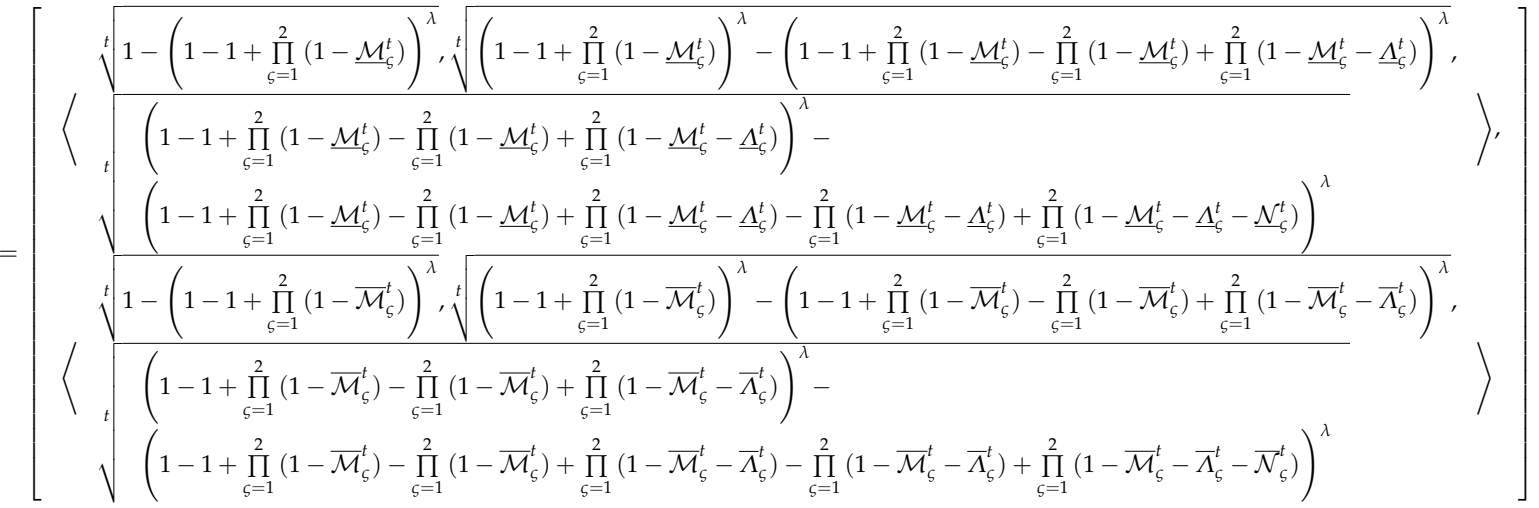




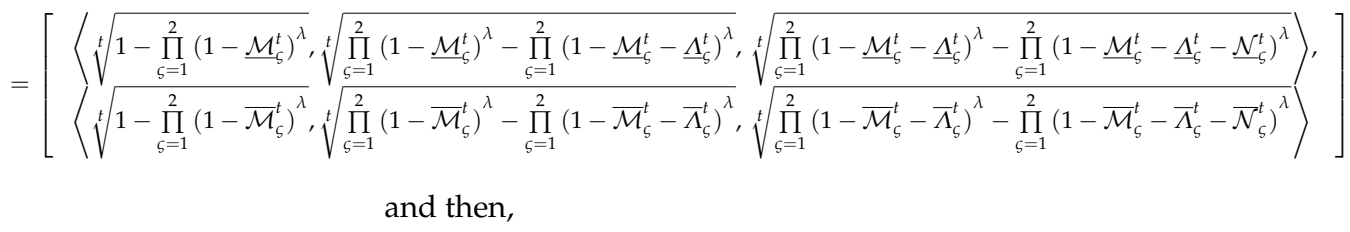

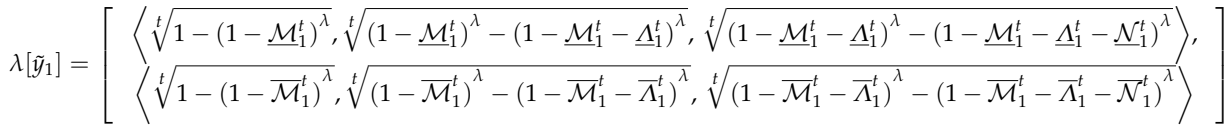

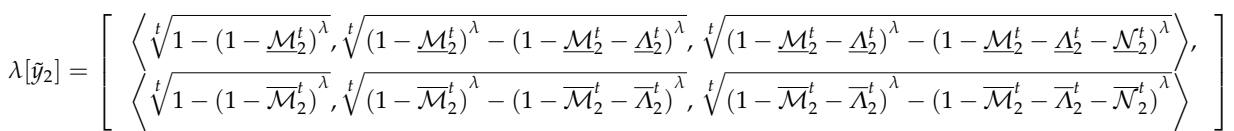

$\lambda\left[\tilde{y}_{1}\right] \oplus \lambda\left[\tilde{y}_{2}\right]$

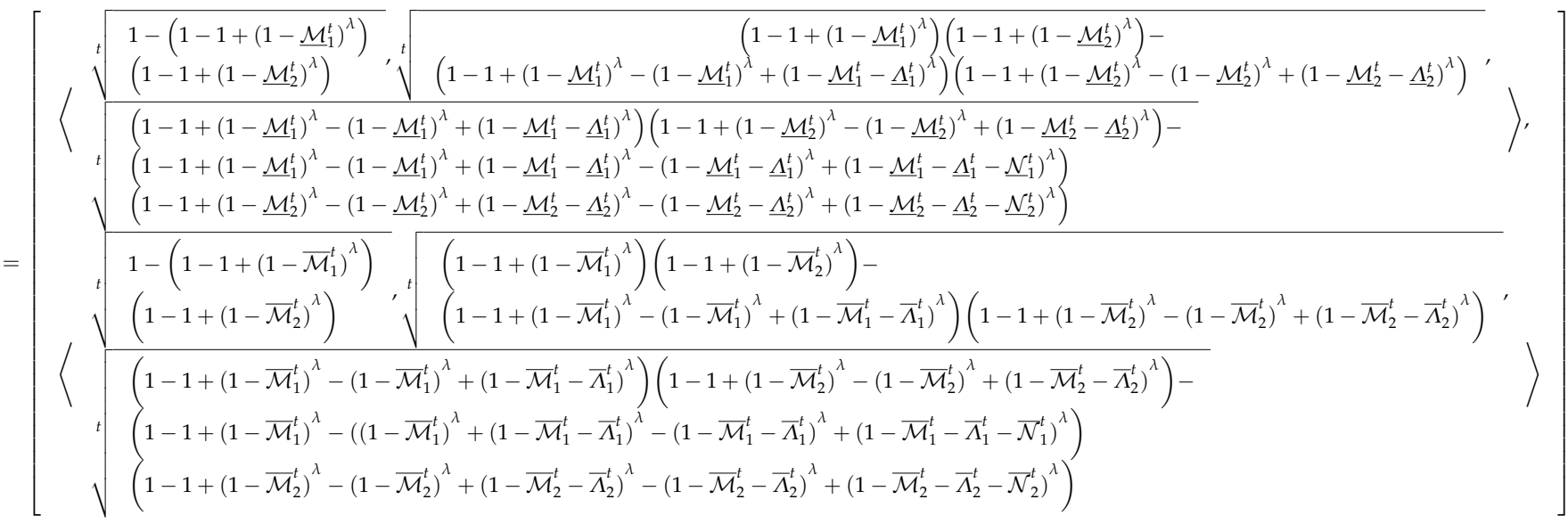

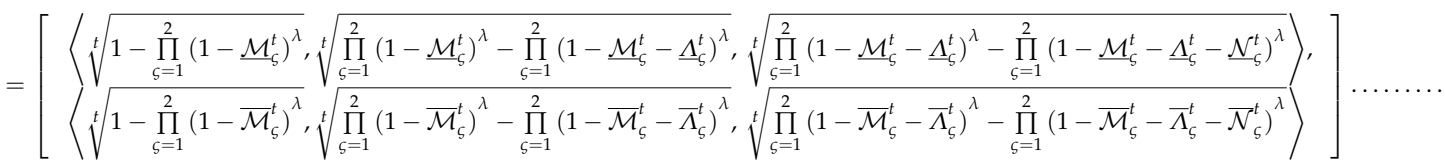

Obviously, (1) = (2), that is, $\lambda([\tilde{y} 1] \oplus[\tilde{y} 2])=\lambda[\tilde{y} 1] \oplus \lambda[\tilde{y} 2](\lambda>0)$. Thus, Property 3 is true. Similarly, Properties 4, 5 and 6 are also true.

Therefore, the proof of Theorem 5 is complete.

\section{The T-SFRIPHM AOs}

\subsection{The T-SFRIPHM Operator}

Definition 17. Let $\left[\tilde{y}_{c}\right](\varsigma=1,2, \ldots, \kappa)$ be a group of T-SFRNs, for any non-negative real number $\eta, \rho$ with $\eta+\rho>0$, if T-SFRIPHM: $\Omega^{\kappa} \rightarrow \Omega$,

$$
T-\operatorname{SFRIPHM} M^{\eta, \rho}\left(\left[\tilde{y}_{1}\right],\left[\tilde{y}_{2}\right], \cdots,\left[\tilde{y}_{\kappa}\right]\right)=\left(\frac{2}{\kappa(\kappa+1)} \sum_{\zeta=1, \xi=\varsigma}^{\kappa}\left(\left(\frac{\kappa\left(1+T\left(\left[\tilde{y}_{\zeta}\right]\right)\right)}{\sum_{v=1}^{\kappa}\left(1+T\left(\left[\tilde{y}_{v}\right]\right)\right)}\left[\tilde{y}_{\zeta}\right]\right)^{\eta} \otimes\left(\frac{\kappa\left(1+T\left(\left[\tilde{y}_{\xi}\right]\right)\right)}{\sum_{v=1}^{\kappa}\left(1+T\left(\left[\tilde{y}_{v}\right]\right)\right)^{\rho}}\left[\tilde{y}_{\xi}\right]\right)^{\rho}\right)\right)^{\frac{1}{\eta+\rho}}
$$

which is called the T-SFRIPHM operator, where $\Omega$ indicates the group of all T-SFRNs, and $\kappa$ is the balance coefficient. $T\left(\left[\tilde{y}_{\zeta}\right]\right)=\sum_{\varsigma=1, \sigma \neq \varsigma}^{\mathcal{k}} \sup \left(\left[\tilde{y}_{\zeta}\right],\left[\tilde{y}_{\sigma}\right]\right)$, where $\sup \left(\left[\tilde{y}_{\varsigma}\right],\left[\tilde{y}_{\sigma}\right]\right)(\varsigma, \sigma=1,2, \ldots, \kappa, \varsigma \neq \sigma)$ indicates the support degree of $\left[\tilde{y}_{\varsigma}\right]$ and $\left[\tilde{y}_{\sigma}\right]$, and it can be obtained from Equation (20):

$$
\sup \left(\left[\tilde{y}_{\varsigma}\right],\left[\tilde{y}_{\sigma}\right]\right)=1-d_{H}\left(\left[\tilde{y}_{\varsigma}\right],\left[\tilde{y}_{\sigma}\right]\right)
$$

where $d_{H}\left(\left[\tilde{y}_{\varsigma}\right],\left[\tilde{y}_{\sigma}\right]\right)$ is denoted as HD between $\left[\tilde{y}_{\varsigma}\right]$ and $\left[\tilde{y}_{\sigma}\right]$, it can be obtained from Equation (16). The value $\sup \left(\tilde{y}_{\zeta}, \tilde{y}_{\sigma}\right)$ features some properties: (1) $\sup \left(\left[\tilde{y}_{\varsigma}\right],\left[\tilde{y}_{\sigma}\right]\right) \in[0,1] ;(2) \sup \left(\left[\tilde{y}_{\varsigma}\right],\left[\tilde{y}_{\sigma}\right]\right)=\sup \left(\left[\tilde{y}_{\sigma}\right],\left[\tilde{y}_{\varsigma}\right]\right)$; (3) if $d_{H}([\tilde{a}],[\tilde{b}])<d_{H}([\tilde{x}],[\tilde{y}])$, then $\sup ([\tilde{a}],[\tilde{b}]) \geq \sup ([\tilde{x}],[\tilde{y}])$. 
Theorem 6. Assuming $\left[\tilde{y}_{\zeta}\right](\varsigma=1,2, \ldots, \kappa)$ is a group of T-SFRNs, for any non-negative real number $\eta$, $\rho$ with $\eta+\rho>0, \omega_{\varsigma}=\left(1+T\left(\left[\tilde{y}_{\zeta}\right]\right)\right) / \sum_{v=1}^{\kappa}\left(1+T\left(\left[\tilde{y}_{v}\right]\right)\right), 0 \leq \omega_{\zeta} \leq 1$ and $\sum_{\zeta=1}^{\kappa} \omega_{\varsigma}=1$, the result from Equation (21) is also a T-SFN.

$$
\begin{aligned}
& T-\operatorname{SFRIPHM}{ }^{\eta, \rho}\left(\left[\tilde{y}_{1}\right],\left[\tilde{y}_{2}\right], \cdots,\left[\tilde{y}_{\kappa}\right]\right)=\left(\frac{2}{\kappa(\kappa+1)} \sum_{\zeta=1, \xi=\varsigma}^{\kappa}\left(\kappa \omega_{\zeta}\left[\tilde{y}_{\zeta}\right]\right)^{\eta} \otimes\left(\kappa \omega_{\xi}\left[\tilde{y}_{\xi}\right]\right)^{\rho}\right)^{\frac{1}{\eta+\rho}} \\
& =\left[\begin{array}{l}
\left\langle\sqrt[t]{(1-\underline{\alpha}+\underline{\beta})^{\frac{1}{\eta+\rho}}-(\underline{\beta})^{\frac{1}{\eta+\rho}}}, \sqrt[t]{(1-\underline{\alpha}+\underline{\beta})^{\frac{1}{\eta+\rho}}-(1-\underline{\gamma}+\underline{\beta})^{\frac{1}{\eta+\rho}}}, \sqrt[t]{1-(1-\underline{\alpha}+\underline{\beta})^{\frac{1}{\eta+\rho}}}\right\rangle, \\
\left\langle\sqrt[t]{(1-\bar{\alpha}+\bar{\beta})^{\frac{1}{\eta+\rho}}-(\bar{\beta})^{\frac{1}{\eta+\rho}}}, \sqrt[t]{(1-\bar{\alpha}+\bar{\beta})^{\frac{1}{\eta+\rho}}-(1-\bar{\gamma}+\bar{\beta})^{\frac{1}{\eta+\rho}}}, \sqrt[t]{1-(1-\bar{\alpha}+\bar{\beta})^{\frac{1}{\eta+\rho}}}\right\rangle
\end{array}\right]
\end{aligned}
$$

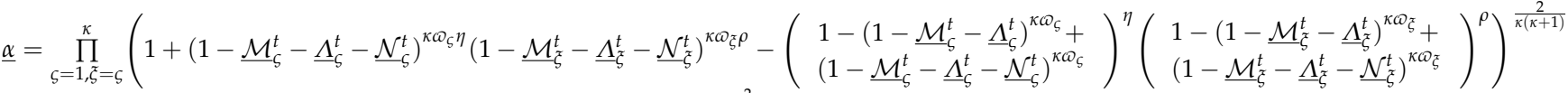

$$
\begin{aligned}
& \underline{\beta}=\prod_{\zeta=1, \xi=\varsigma}^{\kappa}\left(\left(1-\underline{\mathcal{M}}_{\zeta}^{t}-\underline{\Lambda}_{\zeta}^{t}-\underline{\mathcal{N}}_{\zeta}^{t}\right)^{\kappa \omega_{\zeta} \eta}\left(1-\underline{\mathcal{M}}_{\zeta}^{t}-\underline{\Lambda}_{\zeta}^{t}-\underline{\mathcal{N}}_{\tilde{\zeta}}^{t}\right)^{\kappa \omega_{\zeta} \rho}\right)^{\frac{2}{\kappa(\kappa+1)}} \\
& \underline{\gamma}=\prod_{\zeta=1, \xi=\zeta}^{\kappa}\left(1+\left(1-\underline{\mathcal{M}}_{\zeta}^{t}-\underline{\Lambda}_{\zeta}^{t}-\underline{\mathcal{N}}_{\zeta}^{t}\right)^{\kappa \omega_{\zeta} \eta}\left(1-\underline{\mathcal{M}}_{\xi}^{t}-\underline{\Lambda}_{\zeta}^{t}-\underline{\mathcal{N}}_{\zeta}^{t}\right)^{\kappa \omega_{\zeta} \rho}-\left(\begin{array}{c}
1-\left(1-\underline{\mathcal{M}}_{\zeta}^{t}\right)^{\kappa \omega_{\zeta}}+ \\
\left(1-\underline{\mathcal{M}}_{\zeta}^{t}-\underline{\Lambda}_{\zeta}^{t}-\underline{\mathcal{N}}_{\zeta}^{t}\right)^{\kappa \omega_{\zeta}}
\end{array}\right)^{\eta}\left(\begin{array}{c}
1-\left(1-\mathcal{M}_{\xi}^{t}\right)^{\kappa \omega_{\xi}}+ \\
\left(1-\underline{\mathcal{M}}_{\zeta}^{t}-\underline{\Lambda}_{\xi}^{t}-\underline{\mathcal{N}}_{\zeta}^{t}\right)^{\kappa \omega_{\xi}}
\end{array}\right)^{\rho}\right)^{\frac{2}{\kappa(\kappa+1)}}
\end{aligned}
$$

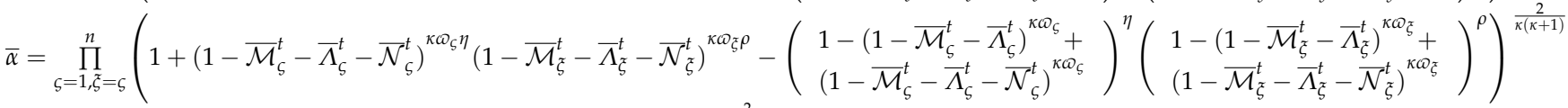

$$
\begin{aligned}
& \bar{\beta}=\prod_{\zeta=1, \xi=\varsigma}^{\kappa}\left(\left(1-\overline{\mathcal{M}}_{\zeta}^{t}-\bar{\Lambda}_{\zeta}^{t}-\overline{\mathcal{N}}_{\zeta}^{t}\right)^{\kappa \omega_{\zeta} \eta}\left(1-\overline{\mathcal{M}}_{\xi}^{t}-\bar{\Lambda}_{\xi}^{t}-\overline{\mathcal{N}}_{\xi}^{t}\right)^{\kappa \omega_{\zeta} \rho}\right)^{\frac{2}{\kappa(\kappa+1)}} \\
& \bar{\gamma}=\prod_{\zeta=1, \xi=\zeta}^{n}\left(1+\left(1-\overline{\mathcal{M}}_{\zeta}^{t}-\bar{\Lambda}_{\zeta}^{t}-\overline{\mathcal{N}}_{\zeta}^{t}\right)^{\kappa \omega_{\zeta} \eta}\left(1-\overline{\mathcal{M}}_{\xi}^{t}-\bar{\Lambda}_{\xi}^{t}-\overline{\mathcal{N}}_{\xi}^{t}\right)^{\kappa \omega_{\xi} \rho}-\left(\begin{array}{c}
1-\left(1-\overline{\mathcal{M}}_{\zeta}^{t}\right)^{\kappa \omega_{\zeta}}+ \\
\left(1-\overline{\mathcal{M}}_{\zeta}^{t}-\bar{\Lambda}_{\zeta}^{t}-\overline{\mathcal{N}}_{\zeta}^{t}\right)^{\kappa \omega_{\zeta}}
\end{array}\right)^{\eta}\left(\begin{array}{c}
1-\left(1-\overline{\mathcal{M}}_{\tilde{\zeta}}^{t}\right)^{\kappa \omega_{\tilde{\xi}}}+ \\
\left(1-\overline{\mathcal{M}}_{\xi}^{t}-\bar{\Lambda}_{\tilde{\zeta}}^{t}-\overline{\mathcal{N}}_{\tilde{\xi}}^{t}\right)^{\kappa \omega_{\xi}}
\end{array}\right)^{\rho}\right)^{\frac{2}{\kappa(\kappa+1)}}
\end{aligned}
$$

Proof. In order to demonstrate that the result from Equation (21) is also a T-SFRN, the TSFRLL and TSFRUL can be proven respectively. To this end, the proof of the TSFRLL is first considered. According to the IOLs in Definition 16, there is

$$
\begin{aligned}
& \kappa \omega_{\zeta}\left[\underline{\tilde{y}}_{\zeta}\right]=\left\langle\left(1-\left(1-\underline{\mathcal{M}}_{\zeta}^{t}\right)^{\kappa \omega_{\zeta}}\right)^{\frac{1}{t}},\left(\left(1-\underline{\mathcal{M}}_{\zeta}^{t}\right)^{\kappa \omega_{\zeta}}-\left(1-\underline{\mathcal{M}}_{\zeta}^{t}-\underline{\Lambda}_{\zeta}^{t}\right)^{\kappa \omega_{\zeta}}\right)^{\frac{1}{t}},\left(\left(1-\underline{\mathcal{M}}_{\zeta}^{t}-\underline{\Lambda}_{\zeta}^{t}\right)^{\kappa \omega_{\zeta}}-\left(1-\underline{\mathcal{M}}_{\zeta}^{t}-\underline{\Lambda}_{\zeta}^{t}-\underline{\mathcal{N}}_{\zeta}^{t}\right)^{\kappa \omega_{\zeta}}\right)^{\frac{1}{t}}\right\rangle \\
& \text { Let } \kappa \omega_{\zeta}\left[\underline{\tilde{y}}_{\zeta}\right]=\left\langle\underline{\mu}_{S^{\prime}} \underline{\eta}_{\zeta^{\prime}} \underline{v}_{\zeta}\right\rangle \text {, then }
\end{aligned}
$$

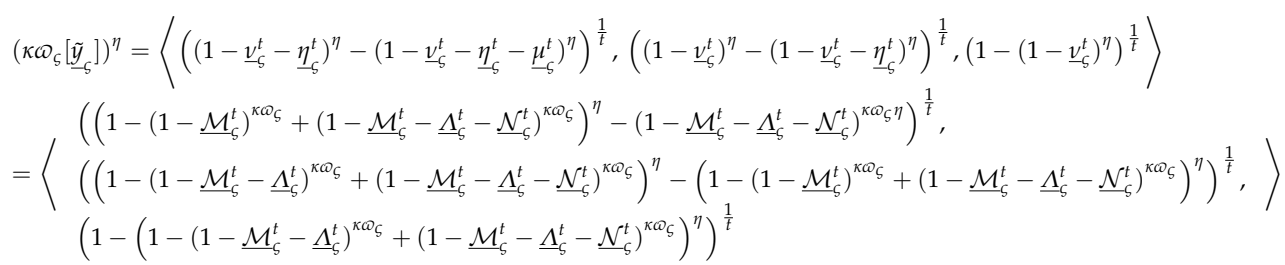$$
\text { Let } a_{\zeta}=1-\left(1-\underline{\mathcal{M}}_{\zeta}^{t}\right)^{\kappa \omega_{\zeta}}+\left(1-\underline{\mathcal{M}}_{\zeta}^{t}-\underline{\Lambda}_{\zeta}^{t}-\underline{\mathcal{N}}_{\zeta}^{t}\right)^{\kappa \omega_{\zeta}}, \quad b_{\zeta}=\left(1-\underline{\mathcal{M}}_{\zeta}^{t}-\underline{\Lambda}_{\zeta}^{t}-\underline{\mathcal{N}}_{\zeta}^{t}\right)^{\kappa \omega_{\zeta}} \text {, }
$$$$
c_{\zeta}=1-\left(1-\underline{\mathcal{M}}_{\zeta}^{t}-\underline{\Lambda}_{\zeta}^{t}\right)^{\kappa \omega_{\zeta}}+\left(1-\underline{\mathcal{M}}_{\zeta}^{t}-\underline{\Lambda}_{\zeta}^{t}-\underline{\mathcal{N}}_{\zeta}^{t}\right)^{\kappa \omega_{\zeta}} \text {, then }\left(\kappa \omega_{\zeta}\left[\underline{\tilde{y}}_{\zeta}\right]\right)^{\eta}=
$$$$
\left\langle\left(a_{\zeta}^{\eta}-b_{\zeta}^{\eta}\right)^{\frac{1}{t}},\left(c_{\zeta}^{\eta}-a_{\zeta}^{\eta}\right)^{\frac{1}{t}},\left(1-c_{\zeta}^{\eta}\right)^{\frac{1}{t}}\right\rangle \text {. Similarly, }\left(\kappa \omega_{\xi}\left[\tilde{y}_{\xi}\right]\right)^{\rho}=\left\langle\left(a_{\xi}^{\rho}-b_{\xi}^{\rho}\right)^{\frac{1}{t}},\left(c_{\xi}^{\rho}-a_{\xi}^{\rho}\right)^{\frac{1}{t}},\left(1-c_{\xi}^{\rho}\right)^{\frac{1}{t}}\right\rangle \text {. }
$$$$
\text { Assume } \underline{\mu}_{\zeta}=\left(a_{\zeta}^{\eta}-b_{\zeta}^{\eta}\right)^{\frac{1}{t}}, \dot{\eta}_{\zeta}=\left(c_{\zeta}^{\eta}-a_{\zeta}^{\eta}\right)^{\frac{1}{t}}, \dot{\underline{v}}_{\zeta}=\left(1-c_{\zeta}^{\eta}\right)^{\frac{1}{t}} \text { and } \underline{\dot{\mu}}_{\xi}=\left(a_{\xi}^{\rho}-b_{\xi}^{\rho}\right)^{\frac{1}{t}} \text {, }
$$$$
\underline{\dot{\eta}}_{\xi}=\left(c_{\xi}^{\rho}-a_{\xi}^{\rho}\right)^{\frac{1}{t}}, \dot{v}_{\xi}=\left(1-c_{\xi}^{\rho}\right)^{\frac{1}{t}} \text {, there is }
$$$$
\left(\kappa \omega_{\varsigma}\left[\tilde{y}_{-}\right]\right)^{\eta} \otimes\left(\kappa \omega_{\xi}\left[\tilde{y}_{\xi}\right]\right)^{\rho}
$$

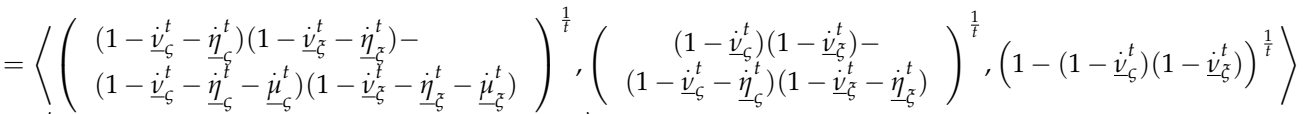$$
=\left\langle\left(a_{\zeta}^{\eta} a_{\xi}^{\rho}-b_{\zeta}^{\eta} b_{\xi}^{\rho}\right)^{\frac{1}{t}},\left(c_{\zeta}^{\eta} c_{\xi}^{\rho}-a_{\zeta}^{\eta} a_{\xi}^{\rho}\right)^{\frac{1}{t}},\left(1-c_{\varsigma}^{\eta} c_{\xi}^{\rho}\right)^{\frac{1}{t}}\right\rangle
$$$$
=\left\langle\underline{\ddot{\mu}}_{\varsigma \xi^{\prime}} \underline{\ddot{\eta}}_{\varsigma \xi^{\prime}} \underline{\ddot{v}}_{\varsigma \xi}\right\rangle
$$ 
Then, if $\kappa=2$, there is

$\sum_{\zeta=1, \tilde{\xi}=\varsigma}^{2}\left(2 \omega_{\zeta}\left[\underline{\tilde{y}}_{-}\right]\right)^{\eta} \otimes\left(2 \omega_{\xi}\left[\underline{\tilde{y}}_{\tilde{\xi}}\right]\right)^{\rho}$

$=\left(\left(2 \omega_{1}\left[\underline{\tilde{y}}_{1}\right]\right)^{\eta} \otimes\left(2 \omega_{1}\left[\underline{\tilde{y}}_{1}\right]\right)^{\rho}\right) \oplus\left(\left(2 \omega_{1}\left[\underline{\tilde{y}}_{1}\right]\right)^{\eta} \otimes\left(2 \omega_{2}\left[\underline{\tilde{y}}_{2}\right]\right)^{\rho}\right) \oplus\left(\left(2 \omega_{2}\left[\underline{\tilde{y}}_{2}\right]\right)^{\eta} \otimes\left(2 \omega_{2}\left[\underline{\tilde{y}}_{2}\right]\right)^{\rho}\right)$

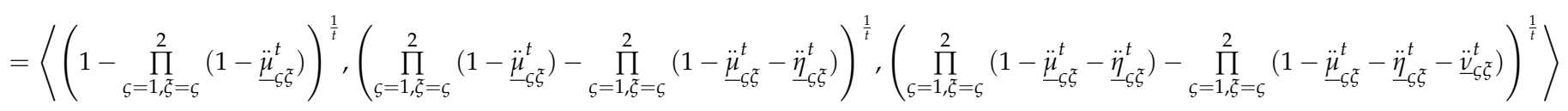

If $\kappa=m$, there is

$$
\begin{aligned}
& \sum_{\zeta=1, \xi=\varsigma}^{m}\left(m \omega_{\zeta}\left[\underline{\tilde{y}}_{\zeta}\right]\right)^{\eta} \otimes\left(m \omega_{\xi}\left[\underline{\tilde{y}}_{\xi}\right]\right)^{\rho}
\end{aligned}
$$

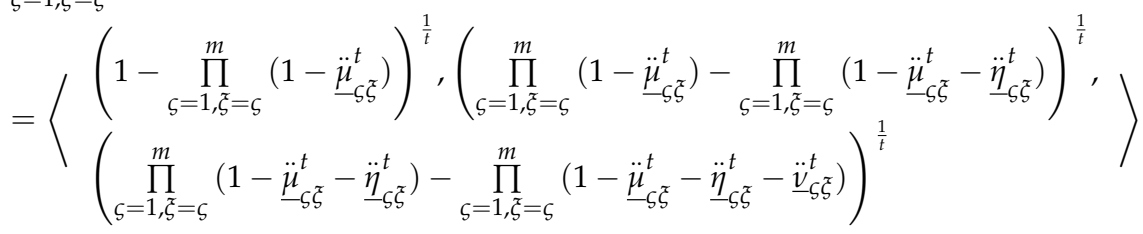

Furthermore, if $\kappa=m+1$, there is

$$
\begin{aligned}
& \sum_{\varsigma=1, \xi^{\xi}=\varsigma}^{m+1}\left((m+1) \boldsymbol{\omega}_{\varsigma}\left[\underline{y}_{\varsigma}\right]\right)^{\eta} \otimes\left((m+1) \boldsymbol{\omega}_{\xi}\left[\tilde{y}_{\tau}\right]\right)^{\rho} \\
& =\left(\sum_{\varsigma=1, \xi=\varsigma}^{m}\left((m+1) \omega_{\varsigma}\left[\underline{y}_{\varsigma}\right]\right)^{\eta} \otimes\left((m+1) \omega_{\xi}\left[\underline{y}_{\xi}\right]\right)^{\rho}\right) \\
& \oplus\left(\left(\sum_{\zeta=1}^{m}\left((m+1) \boldsymbol{\omega}_{\zeta}\left[\underline{\tilde{y}}_{\zeta}\right]\right)^{\eta}\right) \otimes\left((m+1) \boldsymbol{\omega}_{m+1}\left[\underline{\tilde{y}}_{m+1}\right]\right)^{\rho}\right) \\
& \oplus\left(\left((m+1) \omega_{m+1}\left[\underline{\tilde{y}}_{m+1}\right]\right)^{\eta} \otimes\left((m+1) \omega_{m+1}\left[\underline{\tilde{y}}_{m+1}\right]\right)^{\rho}\right)
\end{aligned}
$$

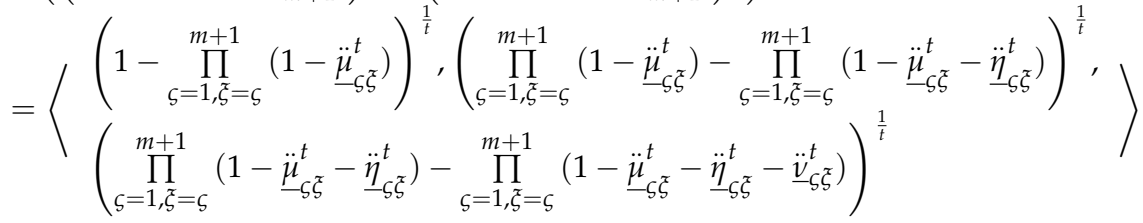

Therefore, the following equation can be obtained through mathematical induction on $\kappa$.

$$
\begin{aligned}
& \sum_{\varsigma=1, \xi=\varsigma}^{\kappa}\left(\kappa \omega_{\zeta}\left[\tilde{y}_{\zeta}\right]\right)^{\eta} \otimes\left(\kappa \omega_{\xi}\left[\tilde{y}_{\tau}\right]\right)^{\rho}
\end{aligned}
$$

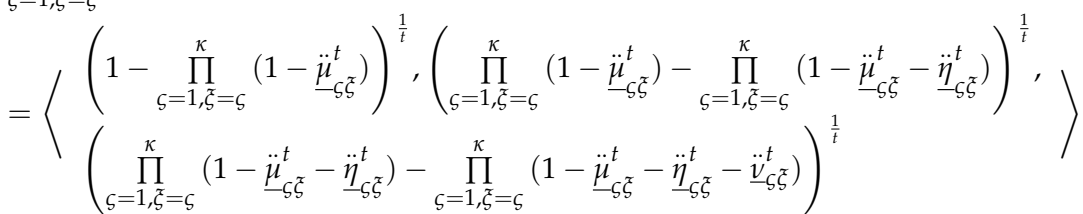

$$
\begin{aligned}
& =\left\langle\begin{array}{l}
\left(1-\prod_{\varsigma=1, \xi=\varsigma}^{\kappa}\left(1-a_{\varsigma}^{\eta} a_{\xi}^{\rho}+b_{\varsigma}^{\eta} b_{\xi}^{\rho}\right)\right)^{\frac{1}{t}},\left(\prod_{\varsigma=1, \xi=\varsigma}^{\kappa}\left(1-a_{\varsigma}^{\eta} a_{\xi}^{\rho}+b_{\varsigma}^{\eta} b_{\xi}^{\rho}\right)-\prod_{\varsigma=1, \xi=\varsigma}^{\kappa}\left(1+b_{\varsigma}^{\eta} b_{\xi}^{\rho}-c_{\varsigma}^{\eta} c_{\xi}^{\rho}\right)\right)^{\frac{1}{t}}, \\
\left(\prod_{\varsigma=1, \xi=\varsigma}^{\kappa}\left(1+b_{\varsigma}^{\eta} b_{\xi}^{\rho}-c_{\zeta}^{\eta} c_{\xi}^{\rho}\right)-\prod_{\varsigma=1, \xi=\varsigma}^{\kappa} b_{\zeta}^{\eta} b_{\xi}^{\rho}\right)^{\frac{1}{t}}
\end{array}\right\rangle \\
& =\left\langle\underline{\ddot{\mu}}_{S \zeta}, \underline{\dddot{\eta}}_{S \zeta}, \underline{\underline{v}}_{S \xi}\right\rangle
\end{aligned}
$$




\section{Further,}

$$
\begin{aligned}
& \frac{2}{\kappa(\kappa+1)} \sum_{\varsigma=1, \xi=\varsigma}^{\kappa}\left(\kappa \omega_{\varsigma}\left[\underline{\tilde{y}}_{\zeta}\right]\right)^{\eta} \otimes\left(\kappa \omega_{\xi}\left[\underline{\tilde{y}}_{\xi}\right]\right)^{\rho}=\frac{2}{\kappa(\kappa+1)} \otimes\left\langle\underline{\ddot{\mu}}_{\varsigma \xi^{\prime}}, \dddot{\dddot{\eta}}_{\varsigma \xi}, \dddot{\underline{v}}_{\varsigma \zeta}\right\rangle
\end{aligned}
$$

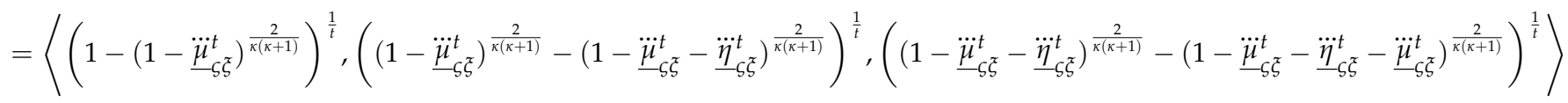

$$
\begin{aligned}
& =\left\langle\begin{array}{l}
\left(1-\prod_{\varsigma=1, \xi=\varsigma}^{\kappa}\left(1-a_{\zeta}^{\eta} a_{\xi}^{\rho}+b_{\zeta}^{\eta} b_{\xi}^{\rho}\right)^{\frac{2}{\kappa(\kappa+1)}}\right)^{\frac{1}{t}},\left(\prod_{\varsigma=1, \xi=\varsigma}^{\kappa}\left(1-a_{\zeta}^{\eta} a_{\xi}^{\rho}+b_{\zeta}^{\eta} b_{\xi}^{\rho}\right)^{\frac{2}{\kappa(\kappa+1)}}-\prod_{\varsigma=1, \xi=\varsigma}^{\kappa}\left(1+b_{\zeta}^{\eta} b_{\xi}^{\rho}-c_{\zeta}^{\eta} c_{\xi}^{\rho}\right)^{\frac{2}{\kappa(\kappa+1)}}\right)^{\frac{1}{\epsilon}}, \\
\left(\prod_{\varsigma=1, \xi=\varsigma}^{\kappa}\left(1+b_{\zeta}^{\eta} b_{\xi}^{\rho}-c_{\zeta}^{\eta} c_{\xi}^{\rho}\right)^{\frac{2}{\kappa(\kappa+1)}}-\prod_{\varsigma=1}^{\kappa}\left(b_{\zeta}^{\eta} b_{\xi}^{\rho}\right)^{\frac{2}{\kappa(\kappa+1)}}\right)^{\frac{1}{t}}
\end{array}\right. \\
& =\left\langle\underline{\dddot{\mu}}_{S \zeta}, \dddot{\dddot{\eta}}_{S \zeta}, \dddot{\underline{v}}_{S \xi}\right\rangle
\end{aligned}
$$

Then,

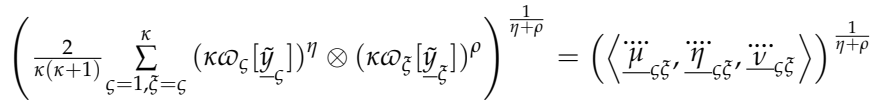

$$
\begin{aligned}
& =\left\langle\left(\left(1-\underline{\dddot{v}}_{i j}^{q}-\underline{\dddot{\eta}}_{i j}^{q}\right)^{\frac{1}{\eta+\rho}}-\left(1-\underline{\dddot{v}}_{i j}^{q}-\underline{\dddot{\eta}}_{i j}^{q}-\underline{\dddot{\mu}}_{i j}^{q}\right)^{\frac{1}{\eta+\rho}}\right)^{\frac{1}{t}},\left(\left(1-\underline{\dddot{v}}_{i j}^{q}\right)^{\frac{1}{\eta+\rho}}-\left(1-\underline{\dddot{w}}_{i j}^{q}-\underline{\dddot{\eta}}_{i j}^{q}\right)^{\frac{1}{\eta+\rho}}\right)^{\frac{1}{t}},\left(1-\left(1-\underline{\dddot{v}}_{i j}^{q}\right)^{\frac{1}{\eta+\rho}}\right)^{\frac{1}{t}}\right\rangle \\
& \left(\left(1-\prod_{\varsigma=1, \tilde{\zeta}=\varsigma}^{\kappa}\left(1+b_{\zeta}^{\eta} b_{\xi}^{\rho}-c_{\zeta}^{\eta} c_{\tilde{\xi}}^{\rho}\right)^{\frac{2}{\kappa^{(\kappa+1)}}}+\prod_{\varsigma=1, \xi=\varsigma}^{\kappa}\left(b_{\zeta}^{\eta} b_{\tilde{\xi}}^{\rho}\right)^{\frac{2}{\kappa(\kappa+1)}}\right)^{\frac{1}{\eta+\rho}}-\prod_{\varsigma=1, \xi=\varsigma}^{\kappa}\left(b_{\zeta}^{\eta} b_{\tilde{\xi}}^{\rho} \frac{2}{)^{\frac{2}{(\kappa+1)}} \cdot \frac{1}{\eta+\rho}}\right)^{\frac{1}{\tau}},\right. \\
& =\left\langle\left(\left(1-\prod_{\varsigma=1, \xi=\varsigma}^{\kappa}\left(1+b_{\zeta}^{\eta} b_{\xi}^{\rho}-c_{\zeta}^{\eta} c_{\xi}^{\rho}\right)^{\frac{2}{\kappa(\kappa+1)}}+\prod_{\varsigma=1, \xi=\varsigma}^{\kappa}\left(b_{\zeta}^{\eta} b_{\xi}^{\rho}\right)^{\frac{2}{\kappa(\kappa+1)}}\right)^{\frac{1}{\eta+\rho}}-\left(1-\prod_{\varsigma=1, \xi=\varsigma}^{\kappa}\left(1-a_{\zeta}^{\eta} a_{\xi}^{\rho}+b_{\zeta}^{\eta} b_{\xi}^{\rho}\right)^{\frac{2}{\kappa(\kappa+1)}}+\prod_{\varsigma=1, \xi=\varsigma}^{\kappa}\left(b_{\zeta}^{\eta} b_{\xi}^{\rho} \frac{2}{)^{\frac{2}{\kappa(\kappa+1)}}}\right)^{\frac{1}{\eta+\rho}}\right)^{\frac{1}{t}},\right\rangle^{\frac{1}{\zeta}}\right. \\
& \left(1-\left(1-\prod_{\varsigma=1, \mathcal{\zeta}=\varsigma}^{\kappa}\left(1+b_{\zeta}^{\eta} b_{\xi}^{\rho}-c_{\zeta}^{\eta} c_{\tilde{\xi}}^{\rho}\right)^{\frac{2}{\kappa(\kappa+1)}}+\prod_{\varsigma=1, \xi=\varsigma}^{\kappa}\left(b_{\zeta}^{\eta} b_{\tilde{\xi}}^{\rho}\right)^{\frac{2}{\kappa(\kappa+1)}}\right)^{\frac{1}{\eta+\rho}}\right)^{\frac{1}{t}} \\
& \begin{array}{l}
\text { Since } a_{\zeta(\xi)}=1-\left(1-\underline{\mathcal{M}}_{\zeta(\xi)}^{t}\right)^{\kappa \omega_{\zeta(\xi)}}+\left(1-\underline{\mathcal{M}}_{\zeta(\xi)}^{t}-\underline{\Lambda}_{\zeta(\xi)}^{t}-\underline{\mathcal{N}}_{\zeta(\xi)}^{t}\right)^{\kappa \omega_{\zeta(\xi)}}, b_{\zeta(\xi)}= \\
\left(1-\underline{\mathcal{M}}_{\zeta(\xi)}^{t}-\underline{\Lambda}_{\zeta(\xi)}^{t}-\underline{\mathcal{N}}_{\zeta(\xi)}^{t}\right)^{\kappa \omega_{\zeta(\xi)}}, \quad c_{\zeta(\xi)}= \\
\left(1-\underline{\mathcal{M}}_{\zeta(\xi)}^{t}-\underline{\Lambda}_{\zeta(\xi)}^{t}-\underline{\mathcal{N}}_{\zeta(\xi)}^{t}\right)^{\kappa \omega_{\zeta(\xi)}} \text {, thus, }
\end{array}
\end{aligned}
$$

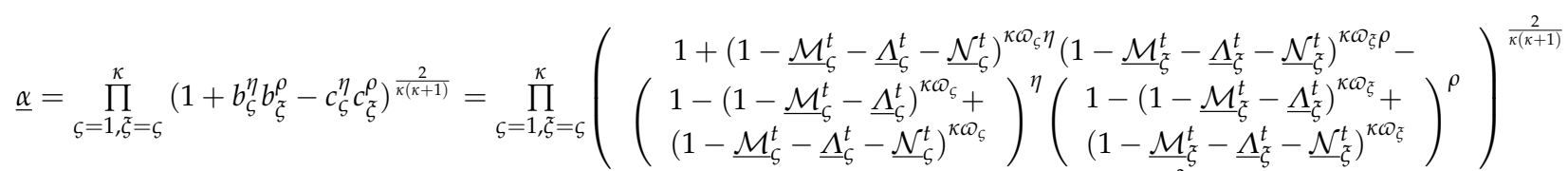$$
\underline{\beta}=\prod_{\zeta=1, \xi=\varsigma}^{\kappa}\left(b_{\zeta}^{\eta} b_{\xi}^{\rho}\right)^{\frac{2}{\kappa(\kappa+1)}}=\prod_{\zeta=1, \xi=\varsigma}^{\kappa}\left(\left(1-\underline{\mathcal{M}}_{\zeta}^{t}-\underline{\Lambda}_{\zeta}^{t}-\underline{\mathcal{N}}_{\zeta}^{t}\right)^{\kappa \omega_{\zeta} \eta}\left(1-\underline{\mathcal{M}}_{\zeta}^{t}-\underline{\Lambda}_{\xi}^{t}-\underline{\mathcal{N}}_{\zeta}^{t}\right)^{\kappa \omega_{\zeta} \rho}\right)^{\frac{2}{\kappa(\kappa+1)}}
$$

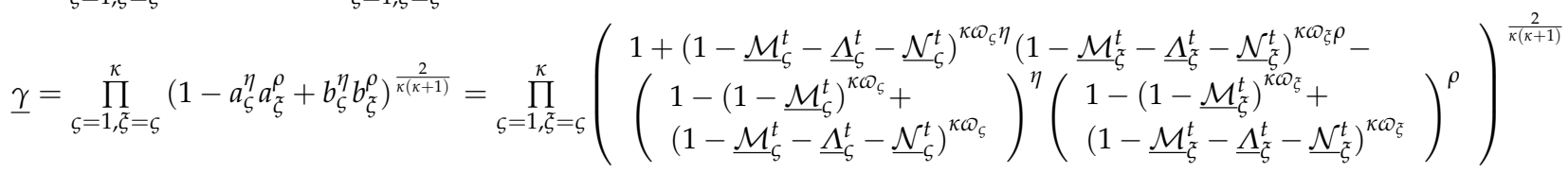

Therefore, the TSFRULL can be obtained as follows,

$$
\left\langle\sqrt[t]{(1-\underline{\alpha}+\underline{\beta})^{\frac{1}{\eta+\rho}}-(\underline{\beta})^{\frac{1}{\eta+\rho}}}, \sqrt[t]{(1-\underline{\alpha}+\underline{\beta})^{\frac{1}{\eta+\rho}}-(1-\underline{\gamma}+\underline{\beta})^{\frac{1}{\eta+\rho}}}, \sqrt[t]{1-(1-\underline{\alpha}+\underline{\beta})^{\frac{1}{\eta+\rho}}}\right\rangle
$$

Similarly, the TSFRUL can be obtained. Therefore, the proof of Theorem 6 is complete.

Example 3. Suppose $\left.\left.\left[\tilde{y}_{1}\right]=[<0.600,0.300,0.600\rangle,<0.700,0.000,0.200\right\rangle\right],\left[\tilde{y}_{2}\right]=[<0.500,0.300,0.700\rangle$, $\left.<0.600,0.600,0.400>],\left[\tilde{y}_{3}\right]=[<0.700,0.400,0.000\rangle,<0.900,0.300,0.200>\right],\left[\tilde{y}_{4}\right]=[<0.400,0.300,0.800\rangle$, 
$<0.500,0.200,0.600>$ ] are four T-SFRNs. The calculation process of Equation (21) is as follows (supposing $t=2$, $\eta=1, \rho=3)$ :

Step 1. Calculated by the Equation (20), the $\sup \left(\left[\tilde{y}_{\varsigma}\right],\left[\tilde{y}_{\sigma}\right]\right)(\varsigma, \sigma=1,2,3,4, \varsigma \neq \sigma)$ is obtained:

$\sup \left(\left[\tilde{y}_{1}\right],\left[\tilde{y}_{2}\right]\right)=\sup \left(\left[\tilde{y}_{2}\right],\left[\tilde{y}_{1}\right]\right)=0.858, \sup \left(\left[\tilde{y}_{1}\right],\left[\tilde{y}_{3}\right]\right)=\sup \left(\left[\tilde{y}_{3}\right],\left[\tilde{y}_{1}\right]\right)=0.838$,

$\sup \left(\left[\tilde{y}_{1}\right],\left[\tilde{y}_{4}\right]\right)=\sup \left(\left[\tilde{y}_{4}\right],\left[\tilde{y}_{1}\right]\right)=0.820, \sup \left(\left[\tilde{y}_{2}\right],\left[\tilde{y}_{3}\right]\right)=\sup \left(\left[\tilde{y}_{3}\right],\left[\tilde{y}_{2}\right]\right)=0.727$,

$\sup \left(\left[\tilde{y}_{2}\right],\left[\tilde{y}_{4}\right]\right)=\sup \left(\left[\tilde{y}_{4}\right],\left[\tilde{y}_{2}\right]\right)=0.855, \sup \left(\left[\tilde{y}_{3}\right],\left[\tilde{y}_{4}\right]\right)=\sup \left(\left[\tilde{y}_{4}\right],\left[\tilde{y}_{3}\right]\right)=0.672$.

Step 2. According to $T\left(\left[\tilde{y}_{\zeta}\right]\right)=\sum_{\zeta=1, \sigma \neq \varsigma}^{\kappa} \sup \left(\left[\tilde{y}_{\zeta}\right],\left[\tilde{y}_{\sigma}\right]\right)$ and $\omega_{\zeta}=\left(1+T\left(\left[\tilde{y}_{\varsigma}\right]\right)\right) /$ $\sum_{v=1}^{\mathcal{K}}\left(1+T\left(\left[\tilde{y}_{v}\right]\right)\right)$, there are

$T\left(\left[\tilde{y}_{1}\right]\right)=2.517, T\left(\left[\tilde{y}_{2}\right]\right)=2.440, T\left(\left[\tilde{y}_{3}\right]\right)=2.237, T\left(\left[\tilde{y}_{4}\right]\right)=2.347$

$\omega_{1}=0.260, \omega_{2}=0.254, \omega_{3}=0.239, \omega_{4}=0.247$.

Step 3. The comprehensive value [y]] is obtained through Equation (21)

$$
\begin{aligned}
& \left.\underline{\alpha}=\prod_{\zeta=1, \xi=\zeta}^{4}\left(\begin{array}{c}
1+\left(1-\underline{\mathcal{M}}_{\zeta}^{2}-\underline{\Lambda}_{\zeta}^{2}-\underline{\mathcal{N}}_{\zeta}^{2}\right)^{4 \times \omega_{\zeta} \times 1}\left(1-\underline{\mathcal{M}}_{\xi}^{2}-\underline{\Lambda}_{\zeta}^{2}-\underline{\mathcal{N}}_{\xi}^{2}\right)^{4 \times \omega_{\xi} \times 3}- \\
\left(1-\left(1-\underline{\mathcal{M}}_{\zeta}^{2}-\underline{\Lambda}_{\zeta}^{2}\right)^{4 \times \omega_{\zeta}}+\right. \\
\left(1-\underline{\mathcal{M}}_{\zeta}^{2}-\underline{\Lambda}_{\zeta}^{2}-\underline{\mathcal{N}}_{\zeta}^{2}\right)^{4 \times \omega_{\zeta}}
\end{array}\right)^{1}\left(\begin{array}{c}
1-\left(1-\underline{\mathcal{M}}_{\zeta}^{2}-\underline{\Lambda}_{\xi}^{2}\right)^{4 \times \omega_{\xi}}+ \\
\left(1-\underline{\mathcal{M}}_{\xi}^{2}-\underline{\Lambda}_{\zeta}^{2}-\underline{\mathcal{N}}_{\xi}^{2}\right)^{4 \times \omega_{\xi}}
\end{array}\right)^{3}\right)^{\frac{2}{4(4+1)}}=0.828 \\
& \underline{\beta}=\prod_{\varsigma=1, \xi=\varsigma}^{4}\left(\left(1-\underline{\mathcal{M}}_{\zeta}^{2}-\underline{\Lambda}_{\zeta}^{2}-\underline{\mathcal{N}}_{\zeta}^{2}\right)^{4 \times \omega_{\zeta} \times 1}\left(1-\underline{\mathcal{M}}_{\xi}^{2}-\underline{\Lambda}_{\xi}^{2}-\underline{\mathcal{N}}_{\xi}^{2}\right)^{4 \times \omega_{\xi} \times 3}\right)^{\frac{2}{4(4+1)}}=0.017 \\
& \left.\underline{\gamma}=\prod_{\zeta=1, \xi=\varsigma}^{4}\left(\begin{array}{c}
1+\left(1-\underline{\mathcal{M}}_{\zeta}^{2}-\underline{\Lambda}_{\zeta}^{2}-\underline{\mathcal{N}}_{\zeta}^{2}\right)^{4 \times \omega_{\zeta} \times 1}\left(1-\underline{\mathcal{M}}_{\zeta}^{2}-\underline{\Lambda}_{\xi}^{2}-\underline{\mathcal{N}}_{\xi}^{2}\right)^{4 \times \omega_{\xi} \times 3}- \\
\left(1-\left(1-\underline{\mathcal{M}}_{\zeta}^{2}\right)^{4 \times \omega_{\zeta}}+\right. \\
\left(1-\underline{\mathcal{M}}_{\zeta}^{2}-\underline{\Lambda}_{\zeta}^{2}-\underline{\mathcal{N}}_{\zeta}^{2}\right)^{4 \times \omega_{\zeta}}
\end{array}\right)^{1}\left(\begin{array}{c}
1-\left(1-\underline{\mathcal{M}}_{\xi}^{2}\right)^{4 \times \omega_{\xi}}+ \\
\left(1-\underline{\mathcal{M}}_{\zeta}^{2}-\underline{\Lambda}_{\xi}^{2}-\underline{\mathcal{N}}_{\xi}^{2}\right)^{4 \times \omega_{\xi}}
\end{array}\right)^{3}\right)^{\frac{2}{4(4+1)}}=0.929 \\
& \text { So, } \underline{\mu_{\tilde{y}}}=\sqrt{(1-0.828+0.017)^{\frac{1}{1+3}}-0.017^{\frac{1}{1+3}}}=0.547 \text {, } \\
& \begin{array}{c}
\underline{\eta_{\tilde{y}}}=\sqrt{(1-0.828+0.017)^{\frac{1}{1+3}}-(1-0.929+0.017)^{\frac{1}{1+3}}}=0.339, \\
\underline{v_{\tilde{y}}}=\sqrt{1-(1-0.828+0.017)^{\frac{1}{1+3}}}=0.584 .
\end{array}
\end{aligned}
$$

Similarly, $\overline{\mu_{\tilde{y}}}=0.662, \overline{\eta_{\tilde{y}}}=0.362, \overline{\nu_{\tilde{y}}}=0.453$.

Thus,

$$
[\tilde{y}]=T-\operatorname{SFRIPHM} M^{1,3}\left(\left[\tilde{y}_{1}\right],\left[\tilde{y}_{2}\right],\left[\tilde{y}_{3}\right],\left[\tilde{y}_{4}\right]\right)=[<0.547,0.339,0.584>,<0.662,0.362,0.453>]
$$

According to Theorem 6, it is found that the proposed operator features the following desirable properties.

1. Idempotency. Suppose $\left[\tilde{y}_{\zeta}\right](\varsigma=1,2, \ldots, \kappa)$ is a family of T-SFRNs, for any non-negative real number $\eta, \rho$ with $\eta+\rho>0$, if $\left[\tilde{y}_{\varsigma}\right]=[\tilde{y}]$, then

$$
T-\operatorname{SFRIPHM} M^{\eta, \rho}\left(\left[\tilde{y}_{1}\right],\left[\tilde{y}_{2}\right], \cdots,\left[\tilde{y}_{\kappa}\right]\right)=[\tilde{y}]
$$

2. Boundedness. Suppose $[\tilde{y} \zeta](\zeta=1,2, \ldots, k)$ is a family of T-SFRNs, for any non- negative real number $\eta$, $\rho$ with $\eta+\rho>0$, then

$$
[\tilde{a}]^{-} \leq T-\operatorname{SFRIPHM} M^{\eta, \rho}\left(\left[\tilde{y}_{1}\right],\left[\tilde{y}_{2}\right], \ldots,\left[\tilde{y}_{\kappa}\right]\right) \leq[\tilde{a}]^{+}
$$

where $[\tilde{a}]^{-}=\left[<\min _{\zeta}\left\{\underline{\mathcal{M}}_{\zeta}\right\}, \max _{\zeta}\left\{\underline{\Lambda}_{\zeta}\right\}, \max _{\zeta}\left\{\underline{\mathcal{N}}_{\zeta}\right\}>,<\min _{\zeta}\left\{\overline{\mathcal{M}}_{\zeta}\right\}, \max _{\zeta}\left\{\bar{\Lambda}_{\zeta}\right\}, \max _{\zeta}\left\{\overline{\mathcal{N}}_{\zeta}\right\}>\right]$, $[\tilde{a}]^{+}=\left[<\max _{\zeta}\left\{\underline{\mathcal{M}}_{\zeta}\right\}, \min _{\zeta}\left\{\underline{\Lambda}_{\zeta}\right\}, \min _{\zeta}\left\{\underline{\mathcal{N}}_{\zeta}\right\}>,<\max _{\zeta}\left\{\overline{\mathcal{M}}_{\zeta}\right\}, \min _{\zeta}\left\{\bar{\Lambda}_{\zeta}\right\}, \min _{\zeta}\left\{\overline{\mathcal{N}}_{\zeta}\right\}>\right]$.

However, the T-SFRIPHM operator does not feature the property of Monotonicity. In accordance with the IOLs of the T-SFRNs in Definition 16, the above properties of the proposed operator can be easily proven, so this proof is omitted here. and $\rho$.

Next, special types of this operator are obtained with regard to different the parameters $t, \eta$ 
Remark 4. Let $\left[\tilde{y}_{\zeta}\right](\varsigma=1,2, \ldots, \kappa)$ be a family of T-SFRNs, when $\eta \rightarrow 0$, the T-SFIPHM operator (Equation (19)) reduces to the T-spherical fuzzy rough interactive power generalized linear ascending weighted operator, i.e.,

$$
T-\operatorname{SFRIPHM} M^{0, \rho}\left(\left[\tilde{y}_{1}\right],\left[\tilde{y}_{2}\right], \cdots,\left[\tilde{y}_{\kappa}\right]\right)=\left(\frac{2}{\kappa(\kappa+1)} \sum_{\zeta=1}^{\kappa} \varsigma\left(\kappa \omega_{\varsigma}\left[\tilde{y}_{\varsigma}\right]\right)^{\rho}\right)^{\frac{1}{\rho}}
$$

where $T-\operatorname{SFRIPHM}{ }^{0, \rho}\left(\left[\tilde{y}_{1}\right],\left[\tilde{y}_{2}\right], \cdots,\left[\tilde{y}_{\kappa}\right]\right)$ can weigh the information $\left(\left(\kappa \omega_{1}\left[\tilde{y}_{1}\right]\right)^{t},\left(\kappa \omega_{2}\left[\tilde{y}_{2}\right]\right)^{t}, \ldots\right.$, $\left.\left(\kappa \omega_{\kappa}\left[\tilde{y}_{\kappa}\right]\right)^{t}\right)$ with weight vector $(1,2, \ldots, \kappa)$. However, Equation (24) cannot reflect the interrelationships between the input T-SFRNs.

Furthermore, If $\eta \rightarrow 0, \rho=1, \omega_{\zeta}=1 / \kappa, t=1$, then Equation (24) reduces to the picture fuzzy rough interaction linear ascending weighted average (PFRILAWA) operator. If $\eta \rightarrow 0, \rho=1, \omega_{\zeta}=1 / \kappa$, $t=2$, then Equation (24) reduces to the spherical fuzzy rough interaction linear ascending weighted average (SFRILAWA) operator. Obviously, the PFRILAWA and SFRILAWA operators can not reflect the correlation between the input variables, nor the overall balance of all the data.

Remark 5. Let $\left[\tilde{y}_{\varsigma}\right](\varsigma=1,2, \ldots, \kappa)$ be a family of T-SFRNs, when $\rho \rightarrow 0$. Equation (19) reduces to the T-spherical fuzzy rough interaction power generalized linear descending weighted operator, namely

$$
T-\operatorname{SFRIPHM} M^{\eta, 0}\left(\left[\tilde{y}_{1}\right],\left[\tilde{y}_{2}\right], \cdots,\left[\tilde{y}_{\kappa}\right]\right)=\left(\frac{2}{\kappa(\kappa+1)} \sum_{\zeta=1}^{\kappa}(\kappa+1-\varsigma)\left(\kappa \omega_{\varsigma}\left[\tilde{y}_{\varsigma}\right]\right)^{\eta}\right)^{\frac{1}{\eta}}
$$

where $T-\operatorname{SFRIPHM} M^{\eta, 0}\left(\left[\tilde{y}_{1}\right],\left[\tilde{y}_{2}\right], \cdots,\left[\tilde{y}_{\kappa}\right]\right)$ can weigh the information $\left(\left(\kappa \omega_{1}\left[\tilde{y}_{1}\right]\right)^{t},\left(\kappa \omega_{2}\left[\tilde{y}_{2}\right]\right)^{t}, \ldots\right.$, $\left.\left(\kappa \omega_{\kappa}\left[\tilde{y}_{\kappa}\right]\right)^{t}\right)$ with weight vector $(\kappa, \kappa-1, \ldots, 1)$. The Equation (25) also cannot reflect the interrelationship between input T-SFRNs.

Similar to Remark 4, if $\rho \rightarrow 0, \eta=1, \omega_{\zeta}=1 / \kappa, t=1$, then Equation (25) reduces to the picture fuzzy rough interaction linear descending weighted average (PFRILDWA) operator. And if $\rho \rightarrow$ $0, \eta=1, \omega_{\zeta}=1 / \kappa, t=2$, the Equation (25) reduces to the spherical fuzzy rough interaction linear descending weighted average (SFRILDWA) operator.

Remark 6. Let $\left[\tilde{y}_{\varsigma}\right](\varsigma=1,2, \ldots, \kappa)$ be a set of T-SFRNs, when $\eta=\rho=1 / 2$. Equation (19) reduces to the T-spherical fuzzy rough interaction power basic HM operator, i.e.,

$$
T-\operatorname{SFRIPHM} M^{\frac{1}{2}, \frac{1}{2}}\left(\left[\tilde{y}_{1}\right],\left[\tilde{y}_{2}\right], \cdots,\left[\tilde{y}_{\kappa}\right]\right)=\frac{2}{\kappa(\kappa+1)} \sum_{\zeta=1, \xi=\varsigma}^{\kappa}\left(\kappa \omega_{\zeta}\left[\tilde{y}_{\zeta}\right]\right)^{\frac{1}{2}} \otimes\left(\kappa \omega_{\xi}\left[\tilde{y}_{\xi}\right]\right)^{\frac{1}{2}}
$$

Similarly, if $\eta=\rho=1 / 2, \omega_{\zeta}=1 / \kappa, t=1$, then Equation (26) reduces to the picture fuzzy rough interaction basic HM operator. Furthermore, if $\eta=\rho=1 / 2, \omega_{\mathcal{S}}=1 / \kappa, t=2$, then Equation (26) reduces to the spherical fuzzy rough basic HM operator.

Remark 7. Let $\left[\tilde{y}_{\varsigma}\right](\varsigma=1,2, \ldots, \kappa)$ be a collective of T-SFRNs, when $\eta=\rho=1$. Equation (19) reduces to the T-spherical fuzzy rough interaction power linear HM operator, i.e.,

$$
T-\operatorname{SFRIPHM} M^{1,1}\left(\left[\tilde{y}_{1}\right],\left[\tilde{y}_{2}\right], \cdots,\left[\tilde{y}_{\kappa}\right]\right)=\left(\frac{2}{\kappa(\kappa+1)} \sum_{\varsigma=1, \xi=\varsigma}^{\kappa}\left(\kappa \omega_{\varsigma}\left[\tilde{y}_{\zeta}\right]\right) \otimes\left(\kappa \omega_{\xi}\left[\tilde{y}_{\xi}\right]\right)\right)^{\frac{1}{2}}
$$

Similarly, if $\eta=\rho=1, \omega_{\mathcal{\zeta}}=1 / \kappa, t=1$, the Equation (27) reduces to the picture fuzzy rough interaction linear HM operator. Furthermore, if $\eta=\rho=1, \omega_{\varsigma}=1 / \kappa, t=2$, the Equation (27) reduces to the spherical fuzzy rough linear HM operator.

\subsection{The T-SFRIPWHM Operator}

Notably, the importance of each input T-SFRN of the T-SFRIPHM operator in Definition 17 is not considered. However, the weight of attribute, as an important input parameter, has a crucial 
part in the aggregation process of attribute variables and can affect the result of AOs in many actual MAGDM problems. Therefore, the T-SFIPWHM operator is further developed.

Definition 18. Suppose $\left[\tilde{y}_{\varsigma}\right](\varsigma=1,2, \ldots, \kappa)$ be a collection of T-SFRNs, for any non-negative real number $\eta, \rho$ with $\eta+\rho>0, \boldsymbol{W}=\left(w_{1}, w_{2}, \ldots, w_{\kappa}\right)^{T}$ is the weight vector of $\left(\left[\tilde{y}_{1}\right],\left[\tilde{y}_{2}\right], \ldots,\left[\tilde{y}_{\kappa}\right]\right)$, meeting $w_{\varsigma} \in[0,1]$ and $\sum_{\zeta=1}^{\kappa} w_{\zeta}=1$, and T-SFRIPHM: $\Omega^{\kappa} \rightarrow \Omega$, if

$T-\operatorname{SFRIPWHM} M_{w}^{\eta, \rho}\left(\left[\tilde{y}_{1}\right],\left[\tilde{y}_{2}\right], \cdots,\left[\tilde{y}_{\kappa}\right]\right)=\left(\frac{2}{\kappa(\kappa+1)} \sum_{\zeta=1, \xi=\varsigma}^{\kappa}\left(\left(\frac{n w_{\zeta}\left(1+T\left(\left[\tilde{y}_{c}\right]\right)\right)}{\left.\sum_{v=1}^{\kappa} w_{v}\left(1+T\left(\tilde{y}_{v}\right]\right)\right)}\left[\tilde{y}_{\zeta}\right]\right)^{\eta} \otimes\left(\frac{\kappa w_{\xi}\left(1+T\left(\left[\tilde{y}_{j}\right]\right)\right)}{\sum_{v=1}^{\kappa} w_{v}\left(1+T\left(\left[\tilde{y}_{v}\right]\right)\right)}\left[\tilde{y}_{j}\right]\right)^{\rho}\right)\right)^{\frac{1}{\eta+\rho}}$

where $\Omega$ is the set of all T-SFRNs, $\kappa$ is the balance coefficient $T\left(\left[\tilde{y}_{\zeta}\right]\right)=\sum_{\zeta=1, \sigma \neq \varsigma}^{\mathcal{N}} \sup \left(\left[\tilde{y}_{\zeta}\right],\left[\tilde{y}_{\sigma}\right]\right)$, where $\sup \left(\left[\tilde{y}_{\varsigma}\right],\left[\tilde{y}_{\sigma}\right]\right)(\varsigma, \sigma=1,2, \ldots, \kappa, \varsigma \neq \sigma)$ is the support degree of $\left[\tilde{y}_{\varsigma}\right]$ and $\left[\tilde{y}_{\sigma}\right]$. Furthermore, $\sup \left(\left[\tilde{y}_{\varsigma}\right]\right.$, $\left.\left[\tilde{y}_{\sigma}\right]\right)$ features the following properties: (1) $\sup \left(\left[\tilde{y}_{\varsigma}\right],\left[\tilde{y}_{\sigma}\right]\right) \in[0,1] ;(2) \sup \left(\left[\tilde{y}_{\varsigma}\right],\left[\tilde{y}_{\sigma}\right]\right)=\sup \left(\left[\tilde{y}_{\sigma}\right],\left[\tilde{y}_{\varsigma}\right]\right) ;(3)$ if $d_{H}([\tilde{a}],[\tilde{b}])<d_{H}([\tilde{x}],[\tilde{y}])$, then $\sup ([\tilde{a}],[\tilde{b}]) \geq \sup ([\tilde{x}],[\tilde{y}])$.

Theorem 7. Supposing $\left[\tilde{y}_{\zeta}\right](\varsigma=1,2, \ldots, \kappa)$ to be a set of T-SFRNs, for any non-negative real number $\eta$, $\rho$ with $\eta+\rho>0, \varepsilon_{\zeta}=\left(\kappa w_{\zeta}\left(1+T\left(\left[\tilde{y}_{\zeta}\right]\right)\right)\right) / \sum_{v=1}^{\kappa} w_{v}\left(1+T\left(\left[\tilde{y}_{v}\right]\right)\right), 0 \leq \varepsilon_{\zeta} \leq 1$ and $\sum_{\zeta=1}^{\mathcal{E}} \varepsilon_{\zeta}=1$, then the aggregated value by using Equation (28) is also a T-SFRN and

$$
\begin{aligned}
& T-\operatorname{SFRIPWHM} M_{w}^{\eta, \rho}\left(\left[\tilde{y}_{1}\right],\left[\tilde{y}_{2}\right], \cdots,\left[\tilde{y}_{\kappa}\right]\right)=\left(\frac{2}{\kappa(\kappa+1)} \sum_{\varsigma=1, \xi=\varsigma}^{\kappa}\left(\varepsilon_{\varsigma}\left[\tilde{y}_{\zeta}\right]\right)^{\eta} \otimes\left(\varepsilon_{\xi}\left[\tilde{y}_{\xi}\right]\right)^{\rho}\right)^{\frac{1}{\eta+\rho}} \\
& =\left[\begin{array}{l}
\left\langle\sqrt[t]{\left(1-\underline{\alpha}_{w}+\underline{\beta}_{w}\right)^{\frac{1}{\eta+\rho}}-\left(\underline{\beta}_{w}\right)^{\frac{1}{\eta+\rho}}}, \sqrt[t]{\left(1-\underline{\alpha}_{w}+\underline{\beta}_{w}\right)^{\frac{1}{\eta+\rho}}-\left(1-\underline{\gamma}_{w}+\underline{\beta}_{w}\right)^{\frac{1}{\eta+\rho}}}, \sqrt[t]{1-\left(1-\underline{\alpha}_{w}+\underline{\beta}_{w}\right)^{\frac{1}{\eta+\rho}}}\right\rangle, \\
\left\langle\sqrt[t]{\left(1-\bar{\alpha}_{w}+\bar{\beta}_{w}\right)^{\frac{1}{\eta+\rho}}-\left(\bar{\beta}_{w}\right)^{\frac{1}{\eta+\rho}}}, \sqrt[t]{\left(1-\bar{\alpha}_{w}+\bar{\beta}_{w}\right)^{\frac{1}{\eta+\rho}}-\left(1-\bar{\gamma}_{w}+\bar{\beta}_{w}\right)^{\frac{1}{\eta+\rho}}}, \sqrt[t]{1-\left(1-\bar{\alpha}_{w}+\bar{\beta}_{w}\right)^{\frac{1}{\eta+\rho}}}\right\rangle
\end{array}\right]
\end{aligned}
$$

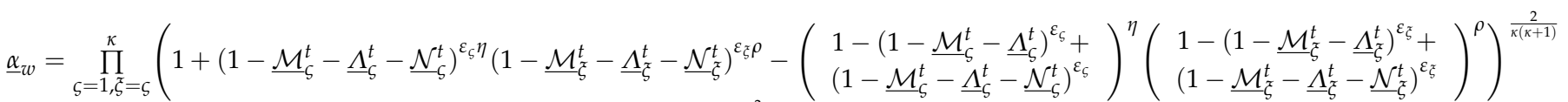

$\underline{\beta}_{w}=\prod_{\zeta=1, \xi=\varsigma}^{\kappa}\left(\left(1-\underline{\mathcal{M}}_{\zeta}^{t}-\underline{\Lambda}_{\zeta}^{t}-\underline{\mathcal{N}}_{\zeta}^{t}\right)^{\varepsilon_{\zeta} \eta}\left(1-\underline{\mathcal{M}}_{\zeta}^{t}-\underline{\Lambda}_{\zeta}^{t}-\underline{\mathcal{N}}_{\zeta}^{t}\right)^{\varepsilon_{\zeta} \rho}\right)^{\frac{2}{\kappa(\kappa+1)}}$

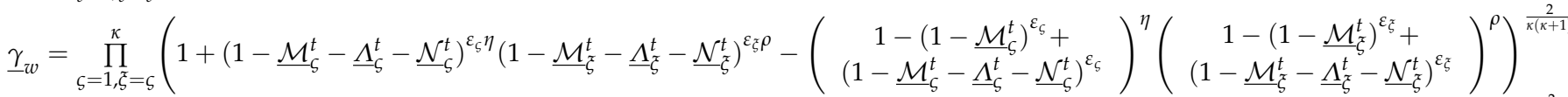

$\bar{\alpha}_{w}=\prod_{\zeta=1, \xi=\varsigma}^{\kappa}\left(1+\left(1-\overline{\mathcal{M}}_{\zeta}^{t}-\bar{\Lambda}_{\zeta}^{t}-\overline{\mathcal{N}}_{\zeta}^{t}\right)^{\varepsilon_{\zeta} \eta}\left(1-\overline{\mathcal{M}}_{\xi}^{t}-\bar{\Lambda}_{\xi}^{t}-\overline{\mathcal{N}}_{\xi}^{t}\right)^{\varepsilon_{\xi} \rho}-\left(\begin{array}{c}1-\left(1-\overline{\mathcal{M}}_{\zeta}^{t}-\bar{\Lambda}_{\zeta}^{t}\right)^{\varepsilon_{\zeta}}+ \\ \left(1-\overline{\mathcal{M}}_{\zeta}^{t}-\bar{\Lambda}_{\zeta}^{t}-\overline{\mathcal{N}}_{\zeta}^{t}\right)^{\varepsilon_{\zeta}}\end{array}\right)^{\eta}\left(\begin{array}{c}1-\left(1-\overline{\mathcal{M}}_{\xi}^{t}-\bar{\Lambda}_{\xi}^{t}\right)^{\varepsilon_{\xi}}+ \\ \left(1-\overline{\mathcal{M}}_{\xi}^{t}-\bar{\Lambda}_{\xi}^{t}-\overline{\mathcal{N}}_{\xi}^{t}\right)^{\varepsilon_{\xi}}\end{array}\right)^{\rho}\right)^{\frac{2}{\kappa^{k(\kappa+1)}}}$

$\bar{\beta}_{w}=\prod_{\varsigma=1, \xi=\varsigma}^{\kappa}\left(\left(1-\overline{\mathcal{M}}_{\zeta}^{t}-\bar{\Lambda}_{\zeta}^{t}-\overline{\mathcal{N}}_{\zeta}^{t}\right)^{\varepsilon_{\zeta} \eta}\left(1-\overline{\mathcal{M}}_{\xi}^{t}-\bar{\Lambda}_{\xi}^{t}-\overline{\mathcal{N}}_{\xi}^{t}\right)^{\varepsilon_{\xi} \rho}\right)^{\frac{2}{\kappa(\kappa+1)}}$

$\bar{\gamma}_{w}=\prod_{\zeta=1, \xi=\varsigma}^{\kappa}\left(1+\left(1-\overline{\mathcal{M}}_{\zeta}^{t}-\bar{\Lambda}_{\zeta}^{t}-\overline{\mathcal{N}}_{\zeta}^{t}\right)^{\varepsilon_{\zeta} \eta}\left(1-\overline{\mathcal{M}}_{\xi}^{t}-\bar{\Lambda}_{\xi}^{t}-\overline{\mathcal{N}}_{\xi}^{t}\right)^{\varepsilon_{\xi} \rho}-\left(\begin{array}{c}1-\left(1-\overline{\mathcal{M}}_{\zeta}^{t}\right)^{\varepsilon_{\zeta}}+ \\ \left(1-\overline{\mathcal{M}}_{\zeta}^{t}-\bar{\Lambda}_{\zeta}^{t}-\overline{\mathcal{N}}_{\zeta}^{t}\right)^{\varepsilon_{\zeta}}\end{array}\right)^{\eta}\left(\begin{array}{c}1-\left(1-\overline{\mathcal{M}}_{\xi}^{t}\right)^{\varepsilon_{\xi}}+ \\ \left(1-\overline{\mathcal{M}}_{\xi}^{t}-\bar{\Lambda}_{\xi}^{t}-\overline{\mathcal{N}}_{\xi}^{t}\right)^{\varepsilon_{\xi}}\end{array}\right)^{\rho}\right)^{\frac{2}{\kappa(k+1)}}$

Following the same proof as Theorem 6, Theorem 7 is true.

This weighted AO reduces to the T-SFRIPHM operator when $w=(1 / \kappa, 1 / \kappa, \ldots, 1 / \kappa)^{T}$, and this AO only satisfies the property of Boundedness.

\section{A Method to MAGDM Based on T-SFRIPWHM Operator}

In this segment, a T-spherical fuzzy MAGDM approach with completely known weights of experts and attributes is proposed. Suppose $\Psi=\left\{\Psi_{1}, \Psi_{2}, \ldots, \Psi_{\kappa}\right\}$ is a finite collection of alternatives and $\boldsymbol{\Phi}=\left\{\Phi_{1}, \Phi_{2}, \ldots, \Phi_{\theta}\right\}$ is a finite collection of attributes, and $\boldsymbol{W}=\left(w_{1}, w_{2}, \ldots, w_{\kappa}\right)^{T}$ is the weight vector of the attributes, meeting $w_{\xi} \geq 0, \sum_{\xi=1}^{\theta} w_{\xi}=1$. Let $\lambda=\left(\lambda_{1}, \lambda_{2}, \ldots, \lambda_{m}\right)^{T}$ be the weight vector of experts, and satisfying $\lambda_{e} \geq 0, \sum_{e=1}^{m} \lambda_{e}=1$. The expert $E_{e}(e=1,2, \ldots, m)$ evaluates the alternative $\Psi_{\zeta}(\zeta=1,2, \ldots, \kappa)$ with respect to the attribute $\Phi_{\xi}(\xi=1,2, \ldots, \theta)$, and the evaluation value is expressed in the form of T-SFNs, namely $\tilde{d}_{\varsigma \xi}^{e}=\left\langle\mathcal{M}_{\varsigma \xi^{\xi}}^{e}, \Lambda_{\varsigma \xi^{\xi}}^{e}, \mathcal{N}_{\varsigma \xi}^{e}\right\rangle$, the individual T-spherical

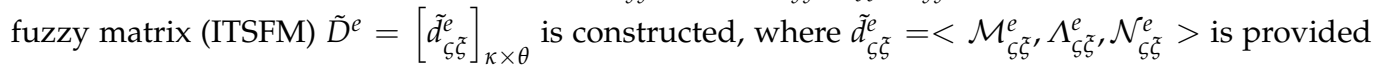
by the $e$-th expert, and $\mathcal{M}_{\zeta \xi^{z}}^{e}, \Lambda_{\zeta \xi^{\prime}}^{e}, \mathcal{N}_{\zeta \xi}^{e}$ respectively represent the MD, AD and NMD, which satisfies $\mathcal{M}_{\zeta \xi}^{e}, \Lambda_{\zeta \xi}^{e}, \mathcal{N}_{\zeta \zeta}^{e} \in[0,1]$ and $\left(\mathcal{M}_{\zeta \xi}^{e}\right)^{t}+\left(\Lambda_{\zeta \xi}^{e}\right)^{t}+\left(\mathcal{N}_{\zeta \zeta}^{e}\right)^{t} \leq 1(e=1,2, \ldots, m ; \zeta=1,2, \ldots, \kappa ; \xi=1,2, \ldots, \theta$; $t \geq 1$ ). 
The T-SFRIPWHM operator is utilized to handle the MAGDM problems in the T-spherical fuzzy environment; the flowchart of proposed method is shown in Figure 1, and the following detailed procedure is presented.

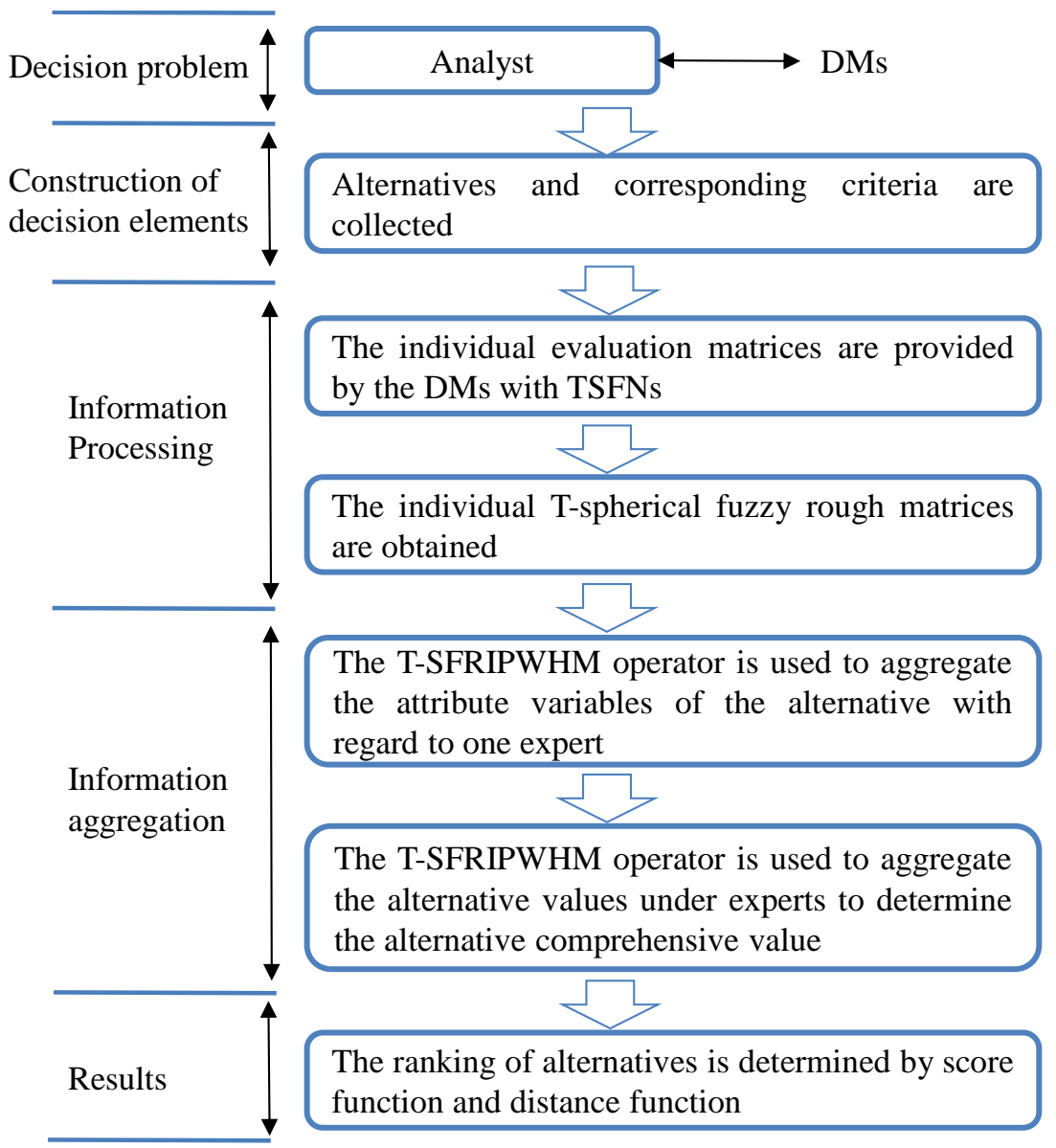

Figure 1. The flowchart of proposed method.

Step 1. It is necessary to guarantee that the types of attributes remain consistent in the decision process, so the cost type attribute is converted into benefit type attribute. Furthermore, the given $\operatorname{ITSFM} \tilde{D}^{e}(e=1,2, \ldots, m)$ can be transformed into a normalized ITSFM $\tilde{R}^{e}=\left[\tilde{r}_{\zeta \xi}^{e}\right]_{\kappa \times \theta}(e=1,2, \ldots, m$; $\varsigma=1,2, \ldots, \kappa ; \xi=1,2, \ldots, \theta)$. The conversion method is presented as

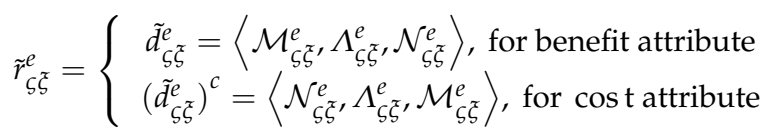

Step 2. According to Equations (12) and (13), the normalized ITSFM $\tilde{R}^{e}$ is transformed into the individual T-spherical fuzzy rough matrix $\left[\tilde{R}^{e}\right]=\left[\left[\tilde{r}_{\zeta \zeta}^{e}\right]\right]_{\mathcal{K} \times \theta^{\prime}}$ where $\left[\tilde{r}_{\zeta \zeta}^{e}\right]=\left[\left\langle\underline{\mathcal{M}}_{\zeta \xi^{\prime}}^{e}, \underline{\Lambda}_{\varsigma \zeta^{\prime}}^{e}, \underline{\mathcal{N}}_{\varsigma \xi}^{e}\right\rangle\right.$, $\left.\left\langle\overline{\mathcal{M}}_{\zeta \xi}^{e}, \bar{\Lambda}_{\zeta \xi}^{e}, \overline{\mathcal{N}}_{\zeta \xi}^{e}\right\rangle\right](e=1,2, \ldots, m ; \varsigma=1,2, \ldots, \kappa ; \xi=1,2, \ldots, \theta)$.

Step 3. Compute the support degree between the T-SFRN $\left[\tilde{r}_{\zeta \xi}^{e}\right]$ and T-SFRN $\left[\tilde{r}_{\zeta \varphi}^{e}\right](\varsigma=1,2, \ldots$, $\kappa ; \xi, \varphi=1,2, \ldots, \theta, \xi \neq \varphi ; e=1,2, \ldots, m)$.

$$
\sup \left(\left[\tilde{r}_{\varsigma \xi}^{e}\right],\left[\tilde{r}_{\xi \varphi}^{e}\right]\right)=1-d_{H}\left(\left[\tilde{r}_{\zeta \xi}^{e}\right],\left[\tilde{r}_{\zeta \varphi}^{e}\right]\right)
$$

where the $d_{H}\left(\left[\tilde{r}_{\zeta \xi}^{e}\right],\left[\tilde{r}_{\zeta \varphi}^{e}\right]\right)$ is the HD between the T-SFRN $\left[\tilde{r}_{\zeta \tau}^{e}\right]$ and T-SFRN $\left[\tilde{r}_{\varsigma \varphi}^{e}\right]$ according to Equation (16).

Step 4. Compute the power weight $\varepsilon_{\zeta \xi}^{e}$ corresponding to the T-SFRN $\left[\tilde{r}_{\zeta \zeta}^{e}\right]$ according to Equations (32) and (33).

$$
T\left(\left[\tilde{r}_{\zeta \xi}^{e}\right]\right)=\sum_{\xi=1, \xi \neq \varphi}^{\kappa} \sup \left(\left[\tilde{r}_{\zeta \xi}^{e}\right],\left[\tilde{r}_{\zeta \varphi}^{e}\right]\right)
$$




$$
\varepsilon_{\zeta \tilde{\zeta}}^{e}==\frac{\kappa w_{\tilde{\mathcal{\zeta}}}\left(1+T\left(\left[\tilde{r}_{\zeta \xi}^{e}\right]\right)\right)}{\sum_{v=1}^{\kappa} w_{v}\left(1+T\left(\left[\tilde{r}_{\zeta v}^{\tilde{\tau}}\right]\right)\right)}
$$

Step 5. Use the T-SFRIPWHM operator to fuse the evaluation information $\left[\tilde{r}_{\varsigma \xi}^{e}\right]$ corresponding to each alternative with respect to the $e$-th expert, and to obtain the comprehensive value $\left[\tilde{g}_{\zeta}^{e}\right]$ ( $\zeta=1$, $2, \ldots, \kappa ; e=1,2, \ldots, m)$ of alternative $\Psi_{\zeta}$.

$$
\left[\tilde{g}_{\varsigma}^{e}\right]=T-\operatorname{SFRIPWHM} M_{w}^{\eta, \rho}\left(\left[\tilde{r}_{\varsigma 1}^{e}\right],\left[\tilde{r}_{\varsigma 2}^{e}\right], \cdots,\left[\tilde{r}_{\varsigma \theta}^{e}\right]\right)
$$

Step 6. Calculate the support degree between the T-SFRN $\left[\tilde{g}_{\zeta}^{e}\right]$ and T-SFRN $\left[\tilde{g}_{\varsigma}^{\delta}\right](\varsigma=1,2, \ldots, \kappa ;$ $e, \delta=1,2, \ldots, m)$

$$
\sup \left(\left[\tilde{g}_{\zeta}^{e}\right],\left[\tilde{g}_{\varsigma}^{\delta}\right]\right)=1-d_{H}\left(\left[\tilde{g}_{\varsigma}^{e}\right],\left[\tilde{g}_{\varsigma}^{\delta}\right]\right)
$$

where the $d_{H}\left(\left[\tilde{g}_{\varsigma}^{e}\right],\left[\tilde{g}_{\varsigma}^{\delta}\right]\right)$ is the HD between the T-SFRN $\left[\tilde{g}_{\varsigma}^{e}\right]$ and T-SFRN $\left[\tilde{g}_{\varsigma}^{\delta}\right]$ according to Equation (16).

Step 7. Calculate the power weight $\varphi_{i}^{\xi}$ corresponding to the T-SFRN $\left[\tilde{g}_{i}^{\xi}\right](\varsigma=1,2, \ldots, \kappa ; \delta=1$, $2, \ldots, m)$ by Equations (36) and (37).

$$
\begin{gathered}
T\left(\left[\tilde{g}_{i}^{\xi}\right]\right)=\sum_{e=1, \delta \neq e}^{m} \sup \left(\left[\tilde{g}_{\zeta}^{e}\right],\left[\tilde{g}_{\zeta}^{\delta}\right]\right) \\
\vartheta_{\zeta}^{e}==\frac{m \lambda_{e}\left(1+T\left(\left[\tilde{g}_{\zeta}^{e}\right]\right)\right)}{\sum_{v=1}^{m} \lambda_{v}\left(1+T\left(\left[\tilde{g}_{\zeta}^{v}\right]\right)\right)}
\end{gathered}
$$

Step 8. The T-SFRIPWHM operator is applied to aggregate the T-SFRN $\left[\tilde{g}_{\zeta}^{e}\right]$ corresponding to each expert, and to obtain the comprehensive value $\left[\tilde{V}_{\zeta}\right]$ of alternative $\Psi_{\zeta}(\zeta=1,2, \ldots, \kappa)$.

$$
\left[\tilde{V}_{\zeta}\right]=T-\operatorname{SFRIPWHM} M_{\lambda}^{\eta, \rho}\left(\left[\tilde{g}_{\zeta}^{1}\right],\left[\tilde{g}_{\zeta}^{2}\right], \cdots,\left[\tilde{g}_{\zeta}^{m}\right]\right)
$$

Step 9. The score function $s c\left(\left[\tilde{V}_{\zeta}\right]\right)$ and distance function $\operatorname{Dis}\left(\left[\tilde{V}_{\zeta}\right]\right)(\varsigma=1,2, \ldots, \kappa)$ of alternative $\Psi_{\zeta}$ are calculated through Equations (17) and (18).

Step 10. The final ranking of alternatives is determined according to Section 3.2, and the best alternation is selected, i.e., the bigger the better.

\section{Numerical Example}

An example is provided to illustrate the application of proposed method in this segment. The following example is revised from Refs. [21,35].

Example 4. Let $\left\{\Psi_{1}, \Psi_{2}, \Psi_{3}, \Psi_{4}\right\}$ be a collection of alternatives, representing the air quality of Guangzhou in 2006, 2007, 2008 and 2009, respectively. $S_{2}\left(\Phi_{1}\right), N_{2}\left(\Phi_{2}\right)$ and $P M_{10}\left(\Phi_{3}\right)$ are used as attributes to describe air quality; their weight values are $0.314,0.355$ and 0.331 , respectively. Suppose there are three air monitoring stations (I, II, III) with weights of 0.4, 0.2 and 0.4, respectively. The experts gave the evaluation values for three stations in regard to the three attributes, and the evaluation information is expressed in the T-SFNs, see Tables 1-3.

Table 1. Air quality data of stations.

\begin{tabular}{ccccc}
\hline Stations & Alternatives & $\boldsymbol{\Phi}_{\mathbf{1}}$ & $\boldsymbol{\Phi}_{\mathbf{2}}$ & $\boldsymbol{\Phi}_{\mathbf{3}}$ \\
\hline$I$ & $\Psi_{1}$ & $<0.265,0.350,0.385>$ & $<0.330,0.390,0.280>$ & $<0.245,0.275,0.480>$ \\
& $\Psi_{2}$ & $<0.345,0.245,0.410>$ & $<0.430,0.290,0.280>$ & $<0.245,0.375,0.380>$ \\
& $\Psi_{3}$ & $<0.365,0.300,0.335>$ & $<0.480,0.315,0.205>$ & $<0.340,0.370,0.290>$ \\
& $\Psi_{4}$ & $<0.430,0.300,0.270>$ & $<0.430,0.300,0.270>$ & $<0.310,0.520,0.170>$ \\
& $\Psi_{1}$ & $<0.125,0.470,0.405>$ & $<0.220,0.420,0.360>$ & $<0.345,0.490,0.165>$ \\
& $\Psi_{2}$ & $<0.335,0.335,0.330>$ & $<0.300,0.370,0.330>$ & $<0.205,0.630,0.165>$ \\
& $\Psi_{3}$ & $<0.250,0.445,0.305>$ & $<0.310,0.585,0.105>$ & $<0.240,0.580,0.220>$ \\
& $\Psi_{4}$ & $<0.365,0.365,0.270>$ & $<0.355,0.320,0.325>$ & $<0.325,0.485,0.190>$ \\
& $\Psi_{1}$ & $<0.325,0.485,0.190>$ & $<0.220,0.450,0.330>$ & $<0.255,0.500,0.245>$ \\
& $\Psi_{2}$ & $<0.270,0.370,0.360>$ & $<0.320,0.215,0.465>$ & $<0.320,0.215,0.465>$ \\
& $\Psi_{3}$ & $<0.510,0.220,0.290>$ & $<0.450,0.370,0.180>$ & $<0.490,0.350,0.160>$ \\
& $\Psi_{4}$ & $<0.390,0.340,0.270>$ & $<0.305,0.475,0.220>$ & $<0.465,0.485,0.050>$ \\
\hline
\end{tabular}


Table 2. T-SFRNs matrix of stations.

\begin{tabular}{|c|c|c|c|c|}
\hline Stations & Alternatives & $\Phi_{1}$ & $\Phi_{2}$ & $\Phi_{3}$ \\
\hline \multirow{8}{*}{$I$} & \multirow{2}{*}{$\Psi_{1}$} & {$[<0.2552,0.3155,0.4347>$} & {$[<0.2828,0.3437,0.3883>$, } & {$[<0.2450,0.2750,0.4800>$, } \\
\hline & & $<0.2996,0.3713,0.3355>]$ & $<0.3300,0.3900,0.2800>]$ & $<0.2828,0.3437,0.3883>]$ \\
\hline & \multirow{2}{*}{$\Psi_{2}$} & {$[<0.3000,0.3161,0.3952>$, } & {$[<0.3504,0.3072,0.3596>$} & {$[<0.2450,0.3750,0.3800>$, } \\
\hline & & $<0.3906,0.2697,0.3492>]$ & $<0.4300,0.2900,0.2800>]$ & $<0.3504,0.3072,0.3596>]$ \\
\hline & \multirow{2}{*}{$\Psi_{3}$} & {$[<0.3528,0.3370,0.3131>$, } & {$[<0.4016,0.3295,0.2798>$} & {$[<0.3400,0.3700,0.2900>$, } \\
\hline & & $<0.4281,0.3084,0.2746>]$ & $<0.4800,0.3150,0.2050>]$ & $<0.4016,0.3295,0.2798>]$ \\
\hline & \multirow{2}{*}{$\Psi_{4}$} & {$[<0.3764,0.4250,0.2232>$} & {$[<0.4070,0.3744,0.2487>$} & {$[<0.3100,0.5200,0.1700>$, } \\
\hline & & $<0.4454,0.2737,0.2828>]$ & $<0.4600,0.2450,0.2950>]$ & $<0.4070,0.3744,0.2487>]$ \\
\hline \multirow{8}{*}{ II } & \multirow{2}{*}{$\Psi_{1}$} & {$[<0.1250,0.4700,0.4050>$, } & {$[<0.1793,0.4456,0.3837>$} & {$[<0.2490,0.4627,0.3234>$, } \\
\hline & & $<0.2490,0.4627,0.3234>]$ & $<0.2905,0.4591,0.2746>]$ & $<0.3450,0.4900,0.1650>]$ \\
\hline & \multirow{2}{*}{$\Psi_{2}$} & {$[<0.2861,0.4718,0.2778>$, } & {$[<0.2575,0.5245,0.2513>$} & {$[<0.2050,0.6300,0.1650>$, } \\
\hline & & $<0.3350,0.3350,0.3300>]$ & $<0.3181,0.3528,0.3300>]$ & $<0.2861,0.4718,0.2778>]$ \\
\hline & \multirow{2}{*}{$\Psi_{3}$} & {$[<0.2451,0.5201,0.2627>$, } & {$[<0.2687,0.5444,0.2192>$} & {$[<0.2400,0.5800,0.2200>$, } \\
\hline & & $<0.2819,0.5254,0.2187>]$ & $<0.3100,0.5850,0.1050>]$ & $<0.2687,0.5444,0.2192>]$ \\
\hline & \multirow{2}{*}{$\Psi_{4}$} & {$[<0.3422,0.3984,0.2648>$, } & {$[<0.3300,0.4142,0.2621>$, } & {$[<0.3250,0.4850,0.1900>$, } \\
\hline & & $<0.3650,0.3650,0.2700>]$ & $<0.3504,0.3438,0.2981>]$ & $<0.3422,0.3984,0.2648>]$ \\
\hline \multirow{8}{*}{ III } & \multirow{2}{*}{$\Psi_{1}$} & {$[<0.2457,0.4601,0.2980>$, } & {$[<0.2200,0.4500,0.3300>$} & {$[<0.2382,0.4764,0.2895>$, } \\
\hline & & $<0.2600,0.4250,0.3150>]$ & $<0.2457,0.4601,0.2980>]$ & $<0.2575,0.4649,0.2811>]$ \\
\hline & \multirow{2}{*}{$\Psi_{2}$} & {$[<0.2144,0.4866,0.3245>$, } & {$[<0.2553,0.4188,0.3739>$} & {$[<0.1350,0.5750,0.2900>$, } \\
\hline & & $<0.2963,0.3033,0.4153>]$ & $<0.3200,0.2150,0.4650>]$ & $<0.2553,0.4188,0.3739>]$ \\
\hline & \multirow{2}{*}{$\Psi_{3}$} & {$[<0.4844,0.3203,0.2169>$, } & {$[<0.4500,0.3700,0.1800>$} & {$[<0.4707,0.3600,0.1701>$, } \\
\hline & & $<0.5100,0.2200,0.2900>]$ & $<0.4844,0.3203,0.2169>]$ & $<0.5002,0.2931,0.2330>]$ \\
\hline & \multirow{2}{*}{$\Psi_{4}$} & {$[<0.3508,0.4130,0.2456>$, } & {$[<0.3050,0.4750,0.2200>$} & {$[<0.3941,0.4421,0.1961>$} \\
\hline & & $<0.4299,0.4259,0.1837>]$ & $<0.3941,0.4421,0.1961>]$ & $<0.4650,0.4850,0.0500>]$ \\
\hline
\end{tabular}

Table 3. Aggregation results of the T-SFRIPWHM operator.

\begin{tabular}{ccccc}
\hline Alternatives & Station I & Station II & Station III \\
\hline \multirow{2}{*}{$\Psi_{1}$} & {$[<0.2777,0.2189,0.3471>$,} & {$[<0.3470,0.3250,0.3098>$,} & {$[<0.3681,0.3355,0.2572>$,} \\
& $<0.3366,0.2691,0.2757>]$ & $<0.4017,0.3515,0.2250>]$ & $<0.3685,0.3293,0.2495>]$ \\
$\Psi_{2}$ & {$[<0.3125,0.2400,0.3059>$,} & {$[<0.4399,0.4100,0.2097>$,} & {$[<0.3816,0.3580,0.2831>$,} \\
& $<0.3436,0.2170,0.2719>]$ & $<0.3552,0.2883,0.2600>]$ & $<0.2975,0.2278,0.3396>]$ \\
\multirow{2}{*}{$\Psi_{3}$} & {$[<0.3585,0.2594,0.2437>$,} & {$[<0.4413,0.4113,0.2091>$,} & {$[<0.4290,0.2823,0.1660>$,} \\
& $<0.3904,0.2477,0.2157>]$ & $<0.4611,0.4226,0.1694>]$ & $<0.4148,0.2272,0.2151>]$ \\
& $\Psi_{4}$ & {$[<0.4194,0.3408,0.1886>$,} & {$[<0.3953,0.3281,0.2054>$,} & {$[<0.4079,0.3347,0.2004>$,} \\
& $<0.3814,0.2340,0.2335>]$ & $<0.3645,0.2771,0.2316>]$ & $<0.4587,0.3564,0.1565>]$ \\
\hline
\end{tabular}

\subsection{The Decision Procedure}

Step 1. Since all attribute types are consistent, no normalization is required here.

Step 2. Convert the T-SFNs in Table 1 into T-SFRNs according to Equations (12) and (13), presented in Table 2.

Step 3. The support degrees $\sup \left(\left[\tilde{r}_{\zeta \xi}^{e}\right],\left[\tilde{r}_{\zeta \varphi}^{e}\right]\right)(\zeta=1,2,3,4 ; \xi, \varphi=1,2,3, \xi \neq \varphi ; e=1,2,3)$ are determined through Equation (31), and for the convenience of denoting $\sup \left(\left[\tilde{r}_{\zeta \xi}^{e}\right],\left[\tilde{r}_{\zeta \varphi}^{e}\right]\right)$ as $S_{\zeta(\xi \varphi)^{e}}^{e}$ then $(t=2)$.

$S_{1(12)}^{1}=S_{1(21)}^{1}=0.9768, S_{1(13)}^{1}=S_{1(31)}^{1}=0.9770, S_{1(23)}^{1}=S_{1(32)}^{1}=0.9538, S_{2(12)}^{1}=S_{2(21)}^{1}=0.9746$,
$S_{2(13)}^{1}=S_{2(31)}^{1}=0.9764, S_{2(23)}^{1}=S_{2(32)}^{1}=0.9588, S_{3(12)}^{1}=S_{3(21)}^{1}=0.9756, S_{3(13)}^{1}=S_{3(31)}^{1}=0.9859$,
$S_{3(23)}^{1}=S_{3(32)}^{1}=0.9676, S_{4(12)}^{1}=S_{4(21)}^{1}=0.9814, S_{4(13)}^{1}=S_{4(31)}^{1}=0.9546, S_{4(23)}^{1}=S_{4(32)}^{1}=0.9360 ;$
$S_{1(12)}^{2}=S_{1(21)}^{2}=0.9816, S_{1(13)}^{2}=S_{1(31)}^{2}=0.9545, S_{1(23)}^{2}=S_{1(32)}^{2}=0.9666, S_{2(12)}^{2}=S_{2(21)}^{2}=0.9824$,
$S_{2(13)}^{2}=S_{2(31)}^{2}=0.9272, S_{2(23)}^{2}=S_{2(32)}^{2}=0.9448, S_{3(12)}^{2}=S_{3(21)}^{2}=0.9702, S_{3(13)}^{2}=S_{3(31)}^{2}=0.9805$,
$S_{3(23)}^{2}=S_{3(32)}^{2}=0.9730, S_{4(12)}^{2}=S_{4(21)}^{2}=0.9894, S_{4(13)}^{2}=S_{4(31)}^{2}=0.9723, S_{4(23)}^{2}=S_{4(32)}^{2}=0.9726 ;$
$S_{1(12)}^{3}=S_{1(21)}^{3}=0.9850, S_{1(13)}^{3}=S_{1(31)}^{3}=0.9865, S_{1(23)}^{3}=S_{1(32)}^{3}=0.9870, S_{2(12)}^{3}=S_{2(21)}^{3}=0.9635$,
$S_{2(13)}^{3}=S_{2(31)}^{3}=0.9531, S_{2(23)}^{3}=S_{2(32)}^{3}=0.9166, S_{3(12)}^{3}=S_{3(21)}^{3}=0.9670, S_{3(13)}^{3}=S_{3(31)}^{3}=0.9774$,
$S_{3(23)}^{3}=S_{3(32)}^{3}=0.9885, S_{4(12)}^{3}=S_{4(21)}^{3}=0.9758, S_{4(13)}^{3}=S_{4(31)}^{3}=0.9674, S_{4(23)}^{3}=S_{4(32)}^{3}=0.9602$.


Step 4. The power weights $\varepsilon_{\zeta \xi}^{e}(\zeta=1,2,3,4 ; \xi=1,2,3 ; e=1,2,3)$ are obtained through Equations (32) and (33).

$$
\begin{aligned}
& \varepsilon_{11}^{1}=0.9471, \varepsilon_{12}^{1}=1.0623, \varepsilon_{13}^{1}=0.9906, \varepsilon_{21}^{1}=0.9457, \varepsilon_{22}^{1}=1.0628, \varepsilon_{23}^{1}=0.9915, \\
& \varepsilon_{31}^{1}=0.9449, \varepsilon_{32}^{1}=1.0617, \varepsilon_{33}^{1}=0.9934, \varepsilon_{41}^{1}=0.9490, \varepsilon_{42}^{1}=1.0661, \varepsilon_{43}^{1}=0.9849 ; \\
& \varepsilon_{11}^{2}=0.9422, \varepsilon_{12}^{2}=1.0696, \varepsilon_{13}^{2}=0.9882, \varepsilon_{21}^{2}=0.9440, \varepsilon_{22}^{2}=1.0737, \varepsilon_{23}^{2}=0.9823, \\
& \varepsilon_{31}^{2}=0.9426, \varepsilon_{32}^{2}=1.0629, \varepsilon_{33}^{2}=0.9945, \varepsilon_{41}^{2}=0.9437, \varepsilon_{42}^{2}=1.0671, \varepsilon_{43}^{2}=0.9892 ; \\
& \varepsilon_{11}^{3}=0.9417, \varepsilon_{12}^{3}=1.0649, \varepsilon_{13}^{3}=0.9934, \varepsilon_{21}^{3}=0.9513, \varepsilon_{22}^{3}=1.0620, \varepsilon_{23}^{3}=0.9867, \\
& \varepsilon_{31}^{3}=0.9385, \varepsilon_{32}^{3}=1.0650, \varepsilon_{33}^{3}=0.9965, \varepsilon_{41}^{3}=0.9445, \varepsilon_{42}^{3}=1.0652, \varepsilon_{43}^{3}=0.9903 .
\end{aligned}
$$

Step 5. Use the T-SFRIPWHM operator to generate $\left[\tilde{g}_{c}^{e}\right](\varsigma=1,2, \ldots, 4 ; e=1,2, \ldots, 3)$. The results are listed in Table 3.

Step 6. Calculate the support degrees $\sup \left(\left[\tilde{g}_{\zeta}^{e}\right],\left[\tilde{g}_{\zeta}^{\delta}\right]\right)(\varsigma=1,2, \ldots, 4 ; e, \delta=1,2, \ldots, 3)$ from Equation (35). For convenience, $\sup \left(\left[\tilde{g}_{\zeta}^{e}\right],\left[\tilde{g}_{\zeta}^{\delta}\right]\right)$ is denoted as $S_{\zeta}^{e \delta}$, and the results are as follows.

$$
\begin{aligned}
& S_{1}^{12}=S_{1}^{21}=0.9583, S_{1}^{13}=S_{1}^{31}=0.9584, S_{1}^{23}=S_{1}^{32}=0.9826, S_{2}^{12}=S_{2}^{21}=0.9489, \\
& S_{2}^{13}=S_{2}^{31}=0.9654, S_{2}^{23}=S_{2}^{32}=0.9599, S_{3}^{12}=S_{3}^{21}=0.9368, S_{3}^{13}=S_{3}^{31}=0.9784, \\
& S_{3}^{23}=S_{3}^{32}=0.9498, S_{4}^{12}=S_{4}^{21}=0.9883, S_{4}^{13}=S_{4}^{31}=0.9691, S_{4}^{23}=S_{4}^{32}=0.9711 .
\end{aligned}
$$

Step 7. Determine the power weights $\vartheta_{\zeta}^{e}(\varsigma=1,2, \ldots, 4 ; e=1,2, \ldots, 3)$ from Equations (36) and (37). The results are as follows.

$$
\begin{aligned}
& \vartheta_{1}^{1}=1.1940, \vartheta_{1}^{2}=0.6020, \vartheta_{1}^{3}=1.2040, \vartheta_{2}^{1}=1.1986, \vartheta_{2}^{2}=0.5982, \vartheta_{2}^{3}=1.2032, \\
& \vartheta_{3}^{1}=1.2002, \vartheta_{3}^{2}=0.5942, \vartheta_{3}^{3}=1.2056, \vartheta_{4}^{1}=1.2026, \vartheta_{4}^{2}=0.6017, \vartheta_{4}^{3}=1.1956
\end{aligned}
$$

Step 8. The T-SFRIPWHM operator is applied to calculate the comprehensive evaluation values $\left[\tilde{V}_{\zeta}\right]$.

$\left[\tilde{V}_{1}\right]=[<0.3122,0.2157,0.2439>,<0.3430,0.2354,0.2068>]$,

$\left[\tilde{V}_{2}\right]=[<0.3554,0.2507,0.2285>,<0.2856,0.1745,0.2332>]$

$\left[\tilde{V}_{3}\right]=[<0.3675,0.2376,0.1722>,<0.3658,0.2249,0.1697>]$,

$\left.\left.\left[\tilde{V}_{4}\right]=[<0.3850,0.2600,0.1643\rangle,<0.3680,0.2296,0.1690\right\rangle\right]$.

\begin{tabular}{|c|c|c|}
\hline$t$ & Score Values & Ranking Order \\
\hline 2 & $\operatorname{sc}\left(\left[\tilde{V}_{1}\right]\right)=0.51595, \operatorname{sc}\left(\left[\tilde{V}_{2}\right]\right)=0.51374, \operatorname{sc}\left(\left[\tilde{V}_{3}\right]\right)=0.55729, \operatorname{sc}\left(\left[\tilde{V}_{4}\right]\right)=0.56013$ & $\Psi_{4} \succ \Psi_{3} \succ \Psi_{1} \succ \Psi_{2}$ \\
\hline 3 & $\operatorname{sc}\left(\left[\tilde{V}_{1}\right]\right)=0.50962, \operatorname{sc}\left(\left[\tilde{V}_{2}\right]\right)=0.51241, \operatorname{sc}\left(\left[\tilde{V}_{3}\right]\right)=0.52741, \operatorname{sc}\left(\left[\tilde{V}_{4}\right]\right)=0.52735$ & $\Psi_{3} \succ \Psi_{4} \succ \Psi_{1} \succ \Psi_{2}$ \\
\hline 4 & $\operatorname{sc}\left(\left[\tilde{V}_{1}\right]\right)=0.50535, \operatorname{sc}\left(\left[\tilde{V}_{2}\right]\right)=0.50828, \operatorname{sc}\left(\left[\tilde{V}_{3}\right]\right)=0.51448, \operatorname{sc}\left(\left[\tilde{V}_{4}\right]\right)=0.51326$ & $\Psi_{3} \succ \Psi_{4} \succ \Psi_{1} \succ \Psi_{2}$ \\
\hline 5 & $\operatorname{sc}\left(\left[\tilde{V}_{1}\right]\right)=0.50370, \operatorname{sc}\left(\left[\tilde{V}_{2}\right]\right)=0.50647, \operatorname{sc}\left(\left[\tilde{V}_{3}\right]\right)=0.50907, \operatorname{sc}\left(\left[\tilde{V}_{4}\right]\right)=0.50795$ & $\Psi_{3} \succ \Psi_{4} \succ \Psi_{1} \succ \Psi_{2}$ \\
\hline 7 & $\operatorname{sc}\left(\left[\tilde{V}_{1}\right]\right)=0.50049, \operatorname{sc}\left(\left[\tilde{V}_{2}\right]\right)=0.50158, \operatorname{sc}\left(\left[\tilde{V}_{3}\right]\right)=0.50176, \operatorname{sc}\left(\left[\tilde{V}_{4}\right]\right)=0.50133$ & $\Psi_{3} \succ \Psi_{2} \succ \Psi_{4} \succ \Psi_{1}$ \\
\hline 10 & $\operatorname{sc}\left(\left[\tilde{V}_{1}\right]\right)=0.50006, \operatorname{sc}\left(\left[\tilde{V}_{2}\right]\right)=0.50032, \operatorname{sc}\left(\left[\tilde{V}_{3}\right]\right)=0.50025, \operatorname{sc}\left(\left[\tilde{V}_{4}\right]\right)=0.50015$ & $\Psi_{2} \succ \Psi_{3} \succ \Psi_{4} \succ \Psi_{1}$ \\
\hline
\end{tabular}

Step 9. The score function $s c\left(\left[\tilde{V}_{\zeta}\right]\right)$ of $\Psi_{\zeta}(\zeta=1,2, \ldots, 4)$ is obtained through Equation (15).

$s c\left(\left[\tilde{V}_{1}\right]\right)=0.5159, s c\left(\left[\tilde{V}_{2}\right]\right)=0.5137, s c\left(\left[\tilde{V}_{3}\right]\right)=0.5572, s c\left(\left[\tilde{V}_{4}\right]\right)=0.5601$

Step 10. According to Definition 12, the ranking of alternatives is $\Psi_{4} \succ \Psi_{3} \succ \Psi_{1} \succ \Psi_{2}$. Thus, the best is $\Psi_{4}$ and the worst is $\Psi_{2}$.

\subsection{Sensitivity Analysis}

\subsubsection{Parameter $t$ Influence Analysis}

The score values and ranking orders are obtained by utilizing the T-SFRIPWHM operator when different parameter $t$. The results are listed in Table 4 .

Table 4. Influence of parameter $t$ on results $(\eta=\rho=1)$.

From Table 4 and Figure 2, the score value of each alternative decreases gradually as the parameter $t$ increases, and the ranking order of alternatives also changes. As can be seen from Table 4 , the alternative $\Psi_{4}$ and alternative $\Psi_{2}$ are the best and worst when $t=2$, respectively. When the $t$ value changes from 3 to 7 , the alternative $\Psi_{3}$ is optimal, while the worst alternative changes from $\Psi_{2}$ to $\Psi_{1}$. When $t=10$, the alternative $\Psi_{2}$ is the most desirable, and $\Psi_{1}$ is the least ideal. 


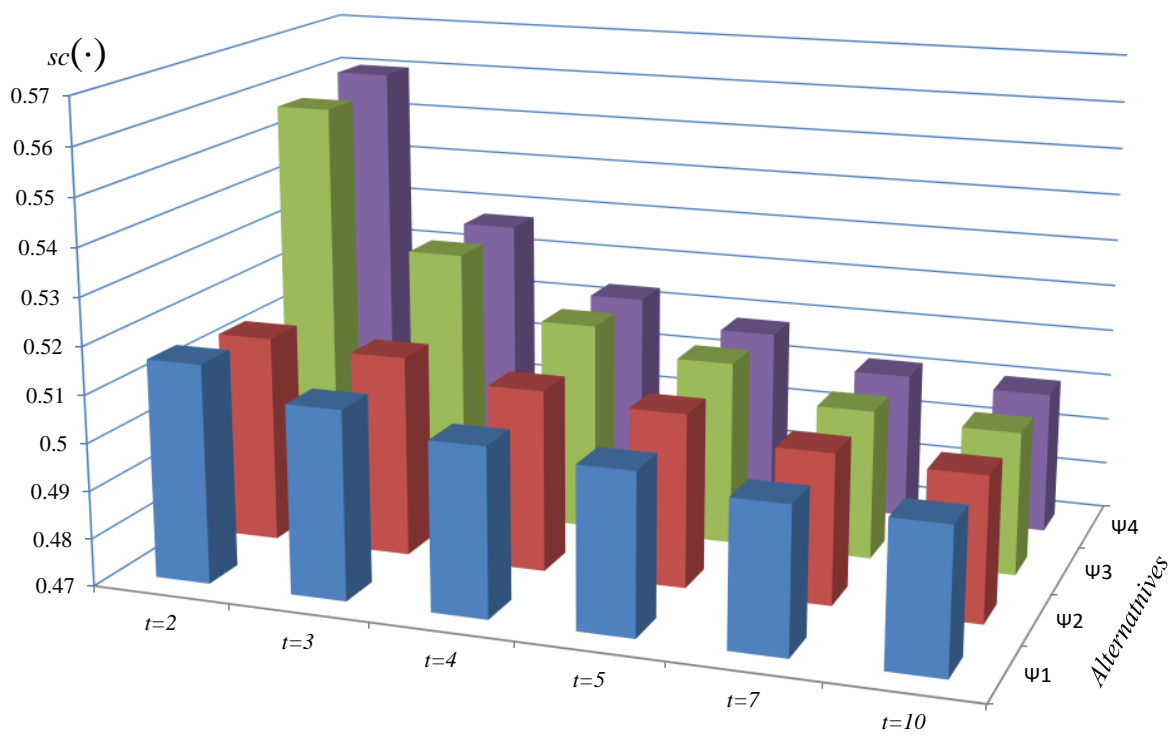

Figure 2. Sensitivity analysis of parameter $t$.

\subsubsection{Parameters $\eta$ and $\rho$ Influence Analysis}

The score values and ranking orders are presented in Table 5, where different values of parameters $\eta$ and $\rho$ are taken in the T-SFRIPWHM operator.

Table 5. Influence of parameters $\eta$ and $\rho$ on the results $(t=2)$.

\begin{tabular}{|c|c|c|}
\hline$\eta, \rho$ & Score Values & Ranking Order \\
\hline 0,1 & $s c\left(\left[\tilde{V}_{1}\right]\right)=0.55787, s c\left(\left[\tilde{V}_{2}\right]\right)=0.54841, s c\left(\left[\tilde{V}_{3}\right]\right)=0.58971, s c\left(\left[\tilde{V}_{4}\right]\right)=0.59767$ & $\Psi_{4} \succ \Psi_{3} \succ \Psi_{1} \succ \Psi_{2}$ \\
\hline 1,0 & $s c\left(\left[\tilde{V}_{1}\right]\right)=0.52171, s c\left(\left[\tilde{V}_{2}\right]\right)=0.52838, s c\left(\left[\tilde{V}_{3}\right]\right)=0.56052, s c\left(\left[\tilde{V}_{4}\right]\right)=0.55924$ & $\Psi_{3} \succ \Psi_{1} \succ \Psi_{2} \succ \Psi_{1}$ \\
\hline 1,1 & $s c\left(\left[\tilde{V}_{1}\right]\right)=0.51595, s c\left(\left[\tilde{V}_{2}\right]\right)=0.51374, s c\left(\left[\tilde{V}_{3}\right]\right)=0.55719, s c\left(\left[\tilde{V}_{4}\right]\right)=0.56013$ & $\Psi_{4} \succ \Psi_{3} \succ \Psi_{1} \succ \Psi_{2}$ \\
\hline 1,3 & $s c\left(\left[\tilde{V}_{1}\right]\right)=0.48585, s c\left(\left[\tilde{V}_{2}\right]\right)=0.47597, s c\left(\left[\tilde{V}_{3}\right]\right)=0.52925, s c\left(\left[\tilde{V}_{4}\right]\right)=0.53377$ & $\Psi_{4} \succ \Psi_{3} \succ \Psi_{1} \succ \Psi_{2}$ \\
\hline 3,1 & $s c\left(\left[\tilde{V}_{1}\right]\right)=0.47460, s c\left(\left[\tilde{V}_{2}\right]\right)=0.47818, s c\left(\left[\tilde{V}_{3}\right]\right)=0.51654, s c\left(\left[\tilde{V}_{4}\right]\right)=0.51652$ & $\Psi_{3} \succ \Psi_{4} \succ \Psi_{1} \succ \Psi_{2}$ \\
\hline 3,3 & $s c\left(\left[\tilde{V}_{1}\right]\right)=0.45319, s c\left(\left[\tilde{V}_{2}\right]\right)=0.44979, s c\left(\left[\tilde{V}_{3}\right]\right)=0.49129, s c\left(\left[\tilde{V}_{4}\right]\right)=0.49409$ & $\Psi_{4} \succ \Psi_{3} \succ \Psi_{1} \succ \Psi_{2}$ \\
\hline 3,5 & $s c\left(\left[\tilde{V}_{1}\right]\right)=0.43650, s c\left(\left[\tilde{V}_{2}\right]\right)=0.43095, s c\left(\left[\tilde{V}_{3}\right]\right)=0.47186, s c\left(\left[\tilde{V}_{4}\right]\right)=0.47578$ & $\Psi_{4} \succ \Psi_{3} \succ \Psi_{1} \succ \Psi_{2}$ \\
\hline 5,5 & $s c\left(\left[\tilde{V}_{1}\right]\right)=0.42339, s c\left(\left[\tilde{V}_{2}\right]\right)=0.42040, s c\left(\left[\tilde{V}_{3}\right]\right)=0.45498, s c\left(\left[\tilde{V}_{4}\right]\right)=0.45832$ & $\Psi_{4} \succ \Psi_{3} \succ \Psi_{1} \succ \Psi_{2}$ \\
\hline 7,7 & $s c\left(\left[\tilde{V}_{1}\right]\right)=0.40914, s c\left(\left[\tilde{V}_{2}\right]\right)=0.40639, s c\left(\left[\tilde{V}_{3}\right]\right)=0.43989, s c\left(\left[\tilde{V}_{4}\right]\right)=0.44443$ & $\Psi_{4} \succ \Psi_{3} \succ \Psi_{1} \succ \Psi_{2}$ \\
\hline 9,9 & $s c\left(\left[\tilde{V}_{1}\right]\right)=0.40159, s c\left(\left[\tilde{V}_{2}\right]\right)=0.39893, s c\left(\left[\tilde{V}_{3}\right]\right)=0.43499, s c\left(\left[\tilde{V}_{4}\right]\right)=0.44102$ & $\Psi_{4} \succ \Psi_{3} \succ \Psi_{1} \succ \Psi_{2}$ \\
\hline
\end{tabular}

With increasing $\eta$ and $\rho$, the score value of each alternative decreases gradually and the ranking results are generally stable at $\Psi_{4} \succ \Psi_{3} \succ \Psi_{1} \succ \Psi_{2}$. In particular, when $\eta=1, \rho=0, \Psi_{3}$ is optimum alternative and the worst alternative is $\Psi_{1}$. When $\eta=3, \rho=1$, the best alternative is also $\Psi_{3}$ and the worst is $\Psi_{2}$.

\subsection{Compare with Existing Methods}

6.3.1. Compare with the Methods without Considering the Balance and Interrelationship

The score values and the ranking of alternatives obtained by different operators without considering the balance and interrelationship under T-spherical fuzzy environment are compared $[21,22,31,33,34,36,37,41]$.

As can be seen in Table 6, the existing methods of the T-SFWA, T-SFWG, T-SFEWA, T-SFEWG, T-SFHWA and T-SFHWG operators all fail to consider the interaction between T-SFNs membership functions, and only take into account the Algebraic, Einstein, and Hamacher operations; the alternative ordering results obtained are all $\Psi_{3} \succ \Psi_{4} \succ \Psi_{2} \succ \Psi_{1}$. In addition, although the T-SFWAI and T-SFWGI operators take the IOLs-J of T-SFNs into consideration, the T-SFWIA and T-SFWGIA consider the IOLs-ZG of T-SFNs. The alternative ordering results obtained by them are consistent, namely $\Psi_{3} \succ \Psi_{4} \succ \Psi_{2} \succ \Psi_{1}$. However, the T-SFDPWA and T-SFDPWG combining attributes' (or experts') priority relationship and the Dombi operator obtain different results, in which the 
T-SFDPWA and proposed method obtain the same optimal alternative, $\Psi_{4}$, while the T-SFDPWG produces the same result as other operators.

Table 6. Comparison with methods without considering equilibrium and interrelationship.

\begin{tabular}{llcl}
\hline AOs & Score Values & Ranking Order \\
\hline T-SFWA $^{1}[21]$ & $s c\left(\left[\tilde{V}_{1}\right]\right)=0.40102, s c\left(\left[\tilde{V}_{2}\right]\right)=0.43084, s c\left(\left[\tilde{V}_{3}\right]\right)=0.50076, s c\left(\left[\tilde{V}_{4}\right]\right)=0.48393$ & $\Psi_{3} \succ \Psi_{4} \succ \Psi_{2} \succ \Psi_{1}$ \\
T-SFWG $^{2}[21,22]$ & $s c\left(\left[\tilde{V}_{1}\right]\right)=0.38997, s c\left(\left[\tilde{V}_{2}\right]\right)=0.41518, s c\left(\left[\tilde{V}_{3}\right]\right)=0.48546, s c\left(\left[\tilde{V}_{4}\right]\right)=0.47048$ & $\Psi_{3} \succ \Psi_{4} \succ \Psi_{2} \succ \Psi_{1}$ \\
T-SFEWA $^{3}[36]$ & $s c\left(\left[\tilde{V}_{1}\right]\right)=0.40002, s c\left(\left[\tilde{V}_{2}\right]\right)=0.42888, s c\left(\left[\tilde{V}_{3}\right]\right)=0.49892, s c\left(\left[\tilde{V}_{4}\right]\right)=0.48246$ & $\Psi_{3} \succ \Psi_{4} \succ \Psi_{2} \succ \Psi_{1}$ \\
T-SFEWG $^{4}[36]$ & $s c\left(\left[\tilde{V}_{1}\right]\right)=0.39032, s c\left(\left[\tilde{V}_{2}\right]\right)=0.41507, s c\left(\left[\tilde{V}_{3}\right]\right)=0.48574, s c\left(\left[\tilde{V}_{4}\right]\right)=0.47022$ & $\Psi_{3} \succ \Psi_{4} \succ \Psi_{2} \succ \Psi_{1}$ \\
T-SFHWA $^{5}[31]$ & $s c\left(\left[\tilde{V}_{1}\right]\right)=0.45273, s c\left(\left[\tilde{V}_{2}\right]\right)=0.47614, s c\left(\left[\tilde{V}_{3}\right]\right)=0.53556, s c\left(\left[\tilde{V}_{4}\right]\right)=0.51951$ & $\Psi_{3} \succ \Psi_{4} \succ \Psi_{2} \succ \Psi_{1}$ \\
T-SFHWG $^{6}[31]$ & $s c\left(\left[\tilde{V}_{1}\right]\right)=0.37115, s c\left(\left[\tilde{V}_{2}\right]\right)=0.38290, s c\left(\left[\tilde{V}_{3}\right]\right)=0.44594, s c\left(\left[\tilde{V}_{4}\right]\right)=0.43263$ & $\Psi_{3} \succ \Psi_{4} \succ \Psi_{2} \succ \Psi_{1}$ \\
T-SFDPWA $^{7}[41]$ & $s c\left(\left[\tilde{V}_{1}\right]\right)=0.39702, s c\left(\left[\tilde{V}_{2}\right]\right)=0.44538, s c\left(\left[\tilde{V}_{3}\right]\right)=0.49968, s c\left(\left[\tilde{V}_{4}\right]\right)=0.55403$ & $\Psi_{4} \succ \Psi_{3} \succ \Psi_{2} \succ \Psi_{1}$ \\
T-SFDPWG $^{8}[41]$ & $s c\left(\left[\tilde{V}_{1}\right]\right)=0.63695, s c\left(\left[\tilde{V}_{2}\right]\right)=0.63771, s c\left(\left[\tilde{V}_{3}\right]\right)=0.72353, s c\left(\left[\tilde{V}_{4}\right]\right)=0.70342$ & $\Psi_{3} \succ \Psi_{4} \succ \Psi_{2} \succ \Psi_{1}$ \\
T-SFWIA $^{9}[33]$ & $s c\left(\left[\tilde{V}_{1}\right]\right)=0.49529, s c\left(\left[\tilde{V}_{2}\right]\right)=0.50284, s c\left(\left[\tilde{V}_{3}\right]\right)=0.58463, s c\left(\left[\tilde{V}_{4}\right]\right)=0.56922$ & $\Psi_{3} \succ \Psi_{4} \succ \Psi_{2} \succ \Psi_{1}$ \\
T-SFWGIA $^{10}[34]$ & $s c\left(\left[\tilde{V}_{1}\right]\right)=0.50454, s c\left(\left[\tilde{V}_{2}\right]\right)=0.53420, s c\left(\left[\tilde{V}_{3}\right]\right)=0.60272, s c\left(\left[\tilde{V}_{4}\right]\right)=0.58766$ & $\Psi_{3} \succ \Psi_{4} \succ \Psi_{2} \succ \Psi_{1}$ \\
T-SFWAI $^{11}[37]$ & $s c\left(\left[\tilde{V}_{1}\right]\right)=0.38886, s c\left(\left[\tilde{V}_{2}\right]\right)=0.40915, s c\left(\left[\tilde{V}_{3}\right]\right)=0.48675, s c\left(\left[\tilde{V}_{4}\right]\right)=0.46558$ & $\Psi_{3} \succ \Psi_{4} \succ \Psi_{2} \succ \Psi_{1}$ \\
T-SFWGI ${ }^{12}[37]$ & $s c\left(\left[\tilde{V}_{1}\right]\right)=0.38779, s c\left(\left[\tilde{V}_{2}\right]\right)=0.40445, s c\left(\left[\tilde{V}_{3}\right]\right)=0.48068, s c\left(\left[\tilde{V}_{4}\right]\right)=0.46359$ & $\Psi_{3} \succ \Psi_{4} \succ \Psi_{2} \succ \Psi_{1}$ \\
T-SFRIPWHM & $s c\left(\left[\tilde{V}_{1}\right]\right)=0.51595, s c\left(\left[\tilde{V}_{2}\right]\right)=0.51374, s c\left(\left[\tilde{V}_{3}\right]\right)=0.55719, s c\left(\left[\tilde{V}_{4}\right]\right)=0.56013$ & $\Psi_{4} \succ \Psi_{3} \succ \Psi_{1} \succ \Psi_{2}$ \\
\hline
\end{tabular}

${ }^{1}$ T-spherical fuzzy weighted average; ${ }^{2}$ T-spherical fuzzy weighted geometric; ${ }^{3}$ T-spherical fuzzy Einstein weighted average; ${ }^{4}$ T-spherical fuzzy Einstein weighted geometric; ${ }^{5}$ T-spherical fuzzy Hamacher weighted average; ${ }^{6}$ T-spherical fuzzy Hamacher weighted geometric; ${ }^{7}$ T-spherical fuzzy Dombi prioritized weighted arithmetic; ${ }^{8}$ T-spherical fuzzy Dombi prioritized weighted geometric; ${ }^{9}$ T-spherical fuzzy weighted interactive averaging; ${ }^{10} \mathrm{~T}$-spherical fuzzy weighted geometric interaction averaging; ${ }^{11} \mathrm{~T}$-spherical fuzzy weighted averaging interaction; ${ }^{12}$ T-spherical fuzzy weighted geometric interaction.

\subsubsection{Comparison with the Methods Considering Equilibrium}

Some T-spherical fuzzy operators $[35,38]$ considering the overall data balance are selected to compare with the proposed operator with T-SFNs.

As can be seen in Table 7, the T-SFPWA and T-SFPWG operators only consider the overall balance of T-SFNs, but do not consider the interrelationship among the attribute variables. This is the same as the alternative ranking results of the T-SFRIPWHM operator in the parameters $\eta=1$, $\rho=0$ and $\eta=0, \rho=1$ scenarios. In the T-SFRIPWHM operator, the overall balance of the T-SFNs and the interrelationship between the attribute variables are both considered when the parameters $\eta=\rho=1$. Obviously, the ranking of the obtained alternatives is more reasonable. In addition, compared with the WT-SFPMM and WT-SFPDMM operators $(\eta=\rho=1)$, the same optimal alternative $\Psi_{4}$ and suboptimal alternative $\Psi_{3}$ are obtained. Obviously, the proposed method takes more account of the IOLs and uncertainty in T-SFSs compared with the WT-SFPMM and WT-SFPDMM operators. Therefore, the T-SFRIPWHM operator is more scientific and advantageous.

Table 7. Comparison with methods with equilibrium.

\begin{tabular}{lccc}
\hline AOs & Score Values & Ranking Order \\
\hline T-SFPWA $^{1}[38]$ & $s c\left(\left[\tilde{V}_{1}\right]\right)=0.73664, s c\left(\left[\tilde{V}_{2}\right]\right)=0.79212, s c\left(\left[\tilde{V}_{3}\right]\right)=0.91189, s c\left(\left[\tilde{V}_{4}\right]\right)=0.88655$ & $\Psi_{3} \succ \Psi_{4} \succ \Psi_{1} \succ \Psi_{2}$ \\
T-SFPWG $^{2}[38]$ & $s c\left(\left[\tilde{V}_{1}\right]\right)=0.15939, s c\left(\left[\tilde{V}_{2}\right]\right)=0.14620, s c\left(\left[\tilde{V}_{3}\right]\right)=0.28635, s c\left(\left[\tilde{V}_{4}\right]\right)=0.29527$ & $\Psi_{4} \succ \Psi_{3} \succ \Psi_{1} \succ \Psi_{2}$ \\
WT-SFPMM $^{3}[35]$ & $s c\left(\left[\tilde{V}_{1}\right]\right)=0.17070, s c\left(\left[\tilde{V}_{2}\right]\right)=0.18064, s c\left(\left[\tilde{V}_{3}\right]\right)=0.24271, s c\left(\left[\tilde{V}_{4}\right]\right)=0.25866$ & $\Psi_{4} \succ \Psi_{3} \succ \Psi_{2} \succ \Psi_{1}$ \\
WT-SFPDMM $^{4}[35]$ & $s c\left(\left[\tilde{V}_{1}\right]\right)=0.41734, s c\left(\left[\tilde{V}_{2}\right]\right)=0.47026, s c\left(\left[\tilde{V}_{3}\right]\right)=0.51831, s c\left(\left[\tilde{V}_{4}\right]\right)=0.52105$ & $\Psi_{4} \succ \Psi_{3} \succ \Psi_{2} \succ \Psi_{1}$ \\
T-SFRIPWHM $(\eta=1, \rho=0)$ & $s c\left(\left[\tilde{V}_{1}\right]\right)=0.52171, s c\left(\left[\tilde{V}_{2}\right]\right)=0.52838, s c\left(\left[\tilde{V}_{3}\right]\right)=0.56052, s c\left(\left[\tilde{V}_{4}\right]\right)=0.55924$ & $\Psi_{3} \succ \Psi_{4} \succ \Psi_{1} \succ \Psi_{2}$ \\
T-SFRIPWHM $(\eta=0, \rho=1)$ & $s c\left(\left[\tilde{V}_{1}\right]\right)=0.55787, s c\left(\left[\tilde{V}_{2}\right]\right)=0.54841, s c\left(\left[\tilde{V}_{3}\right]\right)=0.58971, s c\left(\left[\tilde{V}_{4}\right]\right)=0.59767$ & $\Psi_{4} \succ \Psi_{3} \succ \Psi_{1} \succ \Psi_{2}$ \\
T-SFRIPWHM $(\eta=\rho=1)$ & $s c\left(\left[\tilde{V}_{1}\right]\right)=0.51595, s c\left(\left[\tilde{V}_{2}\right]\right)=0.51374, s c\left(\left[\tilde{V}_{3}\right]\right)=0.55719, s c\left(\left[\tilde{V}_{4}\right]\right)=0.56013$ & $\Psi_{4} \succ \Psi_{3} \succ \Psi_{1} \succ \Psi_{2}$ \\
\hline
\end{tabular}

${ }^{1}$ T-spherical fuzzy power average; ${ }^{2}$ T-spherical fuzzy power geometric; ${ }^{3}$ weighted T-spherical fuzzy power Muirhead mean; ${ }^{4}$ weighted T-spherical fuzzy power dual Muirhead mean.

\subsubsection{Comparison with the Methods Considering Interrelationships}

In the following, three T-spherical fuzzy operators $[30,35]$ considering the interrelationships of attributes are applied to compare with the T-SFRIPWHM operator.

As can be seen in Table 8, the T-SFWGMSM, WT-SFPMM and WT-SFPDMM operators all consider the interrelationship among attribute variables, and the optimal alternative $\Psi_{4}$ and suboptimal one $\Psi_{3}$ are consistent with proposed method, which also reveals the practicability and feasibility of proposed approach. However, the ranking of alternatives $\Psi_{1}$ and $\Psi_{2}$ is different from the proposed 
method, because the T-SFRIPWHM operator considers more uncertainty and interaction than the existing methods described above.

Table 8. Results of comparison of methods with interrelationships.

\begin{tabular}{lcc}
\hline AOs & Score Values & Ranking Order \\
\hline T-SFWGMSM $^{1}[30]$ & $s c\left(\left[\tilde{V}_{1}\right]\right)=0.52187, s c\left(\left[\tilde{V}_{2}\right]\right)=0.53792, s c\left(\left[\tilde{V}_{3}\right]\right)=0.58761, s c\left(\left[\tilde{V}_{4}\right]\right)=0.60224$ & $\Psi_{4} \succ \Psi_{3} \succ \Psi_{2} \succ \Psi_{1}$ \\
WT-SFPMM [35] & $s c\left(\left[\tilde{V}_{1}\right]\right)=0.17070, s c\left(\left[\tilde{V}_{2}\right]\right)=0.18064, s c\left(\left[\tilde{V}_{3}\right]\right)=0.24271, s c\left(\left[\tilde{V}_{4}\right]\right)=0.25866$ & $\Psi_{4} \succ \Psi_{3} \succ \Psi_{2} \succ \Psi_{1}$ \\
WT-SFPDMM [35] & $s c\left(\left[\tilde{V}_{1}\right]\right)=0.41734, s c\left(\left[\tilde{V}_{2}\right]\right)=0.47026, s c\left(\left[\tilde{V}_{3}\right]\right)=0.51831, s c\left(\left[\tilde{V}_{4}\right]\right)=0.52105$ & $\Psi_{4} \succ \Psi_{3} \succ \Psi_{2} \succ \Psi_{1}$ \\
T-SFRIPWHM & $s c\left(\left[\tilde{V}_{1}\right]\right)=0.51595, s c\left(\left[\tilde{V}_{2}\right]\right)=0.51374, s c\left(\left[\tilde{V}_{3}\right]\right)=0.55719, s c\left(\left[\tilde{V}_{4}\right]\right)=0.56013$ & $\Psi_{4} \succ \Psi_{3} \succ \Psi_{1} \succ \Psi_{2}$ \\
\hline
\end{tabular}

${ }^{1}$ T-spherical fuzzy weighted generalized Maclarurin symmetric mean.

Furthermore, characteristics are compared in terms of IOLs, individual and group uncertainty, the overall balance of T-SFNs, and the interrelationships between the attribute variables. The differences among the AOs are listed in Table 9.

Table 9. Comparison of the characteristics of the AOs.

\begin{tabular}{|c|c|c|c|c|c|c|}
\hline AOs & $\begin{array}{c}\text { Individual } \\
\text { and/or Group } \\
\text { Uncertainty }\end{array}$ & $\begin{array}{c}\text { Operation } \\
\text { Rules }\end{array}$ & $\begin{array}{l}\text { Consider IOLs } \\
\text { among } \\
\text { Membership } \\
\text { Functions }\end{array}$ & $\begin{array}{l}\text { Consider the } \\
\text { Equilibrium of } \\
\text { Input } \\
\text { Arguments }\end{array}$ & $\begin{array}{c}\text { Consider } \\
\text { Interrelationship } \\
\text { between } \\
\text { Attributes }\end{array}$ & $\begin{array}{c}\text { Flexibility of } \\
\text { the Information } \\
\text { Process }\end{array}$ \\
\hline T-SFWA [21] & Individual & Algebraic & $\mathrm{NO}$ & $\mathrm{NO}$ & $\mathrm{NO}$ & $\mathrm{NO}$ \\
\hline T-SFWG $[21,22]$ & Individual & Algebraic & $\mathrm{NO}$ & $\mathrm{NO}$ & $\mathrm{NO}$ & $\mathrm{NO}$ \\
\hline T-SFEWA [36] & Individual & Einstein & $\mathrm{NO}$ & NO & $\mathrm{NO}$ & $\mathrm{NO}$ \\
\hline T-SFEWG [36] & Individual & Einstein & $\mathrm{NO}$ & $\mathrm{NO}$ & $\mathrm{NO}$ & $\mathrm{NO}$ \\
\hline T-SFHWA [31] & Individual & Hamacher & $\mathrm{NO}$ & $\mathrm{NO}$ & $\mathrm{NO}$ & Weak \\
\hline T-SFHWG [31] & Individual & Hamacher & $\mathrm{NO}$ & $\mathrm{NO}$ & $\mathrm{NO}$ & Weak \\
\hline T-SFDPWA [41] & Individual & Dombi & $\mathrm{NO}$ & $\mathrm{NO}$ & $\mathrm{NO}$ & Weak \\
\hline T-SFDPWG [41] & Individual & Dombi & $\mathrm{NO}$ & $\mathrm{NO}$ & $\mathrm{NO}$ & Weak \\
\hline T-SFWIA [33] & Individual & IOLs-ZG & YES & NO & $\mathrm{NO}$ & NO \\
\hline T-SFWGIA [34] & Individual & IOLs-ZG & YES & $\mathrm{NO}$ & $\mathrm{NO}$ & $\mathrm{NO}$ \\
\hline T-SFWAI [37] & Individual & IOLs-J & YES & $\mathrm{NO}$ & $\mathrm{NO}$ & $\mathrm{NO}$ \\
\hline T-SFWGI [37] & Individual & IOLs-J & YES & $\mathrm{NO}$ & $\mathrm{NO}$ & $\mathrm{NO}$ \\
\hline T-SFPWA [38] & Individual & Algebraic & $\mathrm{NO}$ & YES & $\mathrm{NO}$ & $\mathrm{NO}$ \\
\hline T-SFPWG [38] & Individual & Algebraic & NO & YES & NO & NO \\
\hline T-SFWGMSM [30] & Individual & Algebraic & $\mathrm{NO}$ & NO & YES & Strong \\
\hline WT-SFPMM [35] & Individual & Algebraic & $\mathrm{NO}$ & YES & YES & Strong \\
\hline WT-SFPDMM [35] & Individual & Algebraic & $\mathrm{NO}$ & YES & YES & Strong \\
\hline T-SFRIPWHM & Both & IOLs-J & YES & YES & YES & Strong \\
\hline
\end{tabular}

In summary, the superiorities of the T-SFRIPWHM operator are as follows:

- The concept of T-SFRN can handle both individual uncertainty and group uncertainty in Tspherical fuzzy MAGDM problems, which ensures the integrity of evaluation information and makes the decision results more reasonable. Other existing methods only consider the individual uncertainty of the individual decision maker, so that reasonable decision results cannot be obtained in some group decision situations.

- $\quad$ The IOLs of T-SFTN is extended based on IOLs- $J$; that is, the interaction among the MD, AD and NMD in the T-SFRLL and T-SFRUL is considered respectively, so as to avoid the irrational decision results when the MD, AD or NMD is 0 in the aggregated values of the T-SFRIPHM AOs.

- $\quad$ The PA operator is included in the T-SFRIPHM AOs, which can effectively reduce the influence of the abnormal preference evaluation value given by the decision-maker due to personal emotion or insufficient understanding of the decision objects. Thus, it can improve the controllability and fairness of the decision-making process.

- The HM operator can reflect the interrelationships between the attribute variables in the proposed T-SFRIPHM OAs. Compared with the existing methods without considering the relationship between the variables, the T-SFRIPHM AOs can consider more evaluation information in the actual decision process.

- $\quad$ The proposed T-SFRIPHM AOs integrate the advantages of RNs, IOLs, PA and HM. In the real-life decision-making process, the proposed method can comprehensively consider the uncertainty of individuals and groups, the interaction between membership functions, the overall balance of input T-SFNs, and the interrelationships between input arguments. 
Therefore, it is more suitable for dealing with complex MAGDM problems in the T-spherical fuzzy environment.

\section{Conclusions}

In this study, a new MAGDM model based on the T-SFRIPWHM operator was developed and applied to solve group decision-making problems with T-spherical fuzzy information. The main conclusions are as follows:

- To deal with the uncertainty of expert individuals and expert groups in group decision-making, a new concept T-SFRN was constructed. At the same time, the distance measure and ordering rules of The -SFRNs and the IOLs of T-SFRNs were extended to eliminate the counterintuitive phenomenon.

- To guarantee the integrity and rationality of evaluation information, and to effectively extract the interrelationship between T-spherical fuzzy variables and the overall information about decision objects, T-SFRIPHM and T-SFRIPWHM operators were proposed from a multi-dimensional perspective, which integrates the advantages of RNs, IOLs, PA and HM. These operators cannot only deal with the uncertainty of individual and group decision makers at the same time, but also consider the interaction between membership functions in T-SFNs, and can reflect the interrelationship between aggregation variables and the overall equilibrium of aggregation T-SFNs, so as reduce the interference of "singularity" as much as possible and make the decisionmaking process more objective and fair.

- $\quad$ A new approach for dealing with T-spherical fuzzy MAGDM problems based on the T-SFRIPWHM operator was developed. Through the application and analysis of example, the effectiveness and feasibility of the proposed approach were shown.

However, the proposed method also features three weaknesses: (1) although the amount of computation of the T-SFRIPWHM operator is higher than that of existing AOs, it can accurately obtain the calculation results and meets the complexity of the actual decision-making problems. (2) The AOs proposed cannot satisfy Monotonicity and other properties. We will try to consider operation laws containing parameters, such as Frank operations, so that these AOs can satisfy relevant properties with respect to this parameter. (3) The attributes weight vector is assumed rather than obtained through a particular approach. In the follow-up study, we will adopt subjective, objective or combined weighting methods to obtain attribute weights according to the actual conditions and requirements of T-spherical fuzzy decision-making problems.

In the future, our proposed AOs can be further extended to different decision-making environments, such as simplified Neutrosophic sets [68], hesitant fuzzy sets [69], and uncertain linguistic sets [70]. We will focus on the integration of the proposed AOs with other sorting methods (e.g., TOPSIS, VIKOR, WASPAS, MARCOS, etc.) and apply them to different real-life decision problems.

Funding: This research was funded by the Humanities and Social Science Foundation of Ministry of Education of the People's Republic of China (No. 19YJC630164) and the Postdoctoral Science Foundation of Jiangxi Province (No. 2019KY14).

Institutional Review Board Statement: Not applicable.

Informed Consent Statement: Not applicable.

Data Availability Statement: Not applicable.

Conflicts of Interest: The authors declare no conflict of interest.

\section{References}

1. Zadeh, L.A. Fuzzy sets. Inf. Control. 1965, 8, 338-353. [CrossRef]

2. Atanassov, K.T. Intuitionistic fuzzy sets. Fuzzy Sets Syst. 1986, 20, 87-96. [CrossRef]

3. Yager, R.R. Pythagorean membership grades in multicriteria decision making. IEEE Trans. Fuzzy Syst. 2014, $22,958-965$. [CrossRef]

4. Yager, R.R. Pythagorean fuzzy subsets. In Proceedings of the 2013 Joint IFSA World Congress and NAFIPS Annual Meeting (IFSA/NAFIPS), Edmonton, AB, Canada, 24-28 June 2013; pp. 57-61.

5. Senapati, T.; Yager, R.R. Fermatean fuzzy sets. J. Ambient Intell. Humaniz. Comput. 2020, 11, 663-674. [CrossRef]

6. Senapati, T.; Yager, R.R. Some new operations over Fermatean fuzzy numbers and application of Fermatean fuzzy wpm in multiple criteria decision making. Informatica 2019, 30, 391-412. [CrossRef]

7. Yager, R.R. Generalized orthopair fuzzy sets. IEEE Trans. Fuzzy Syst. 2017, 25, 1222-1230. [CrossRef] 
8. Yang, Z.L.; He, P. A decision algorithm for selecting the design scheme for blockchain-based agricultural product traceability system in $q$-rung orthopair fuzzy environment. J. Clean. Prod. 2021, 290, 125191. [CrossRef]

9. Wei, G.W.; Wei, C.; Gao, H.; Wei, Y. Some $q$-rung orthopair fuzzy maclaurin symmetric mean operators and their applications to potential evaluation of emerging technology commercialization. Int. J. Intell. Syst. 2019, 34, 50-81. [CrossRef]

10. Krishankumar, R.; Nimmagadda, S.S.; Rani, P.; Mishra, A.R.; Ravichandran, K.S.; Gandomi, A.H. Solving renewable energy source selection problems using a $q$-rung orthopair fuzzy-based integrated decision-making approach. J. Clean. Prod. 2021, 279, 123329. [CrossRef]

11. Pinar, A.; Rouyendegh, B.D.; Ozdemir, Y.S. q-rung orthopair fuzzy TOPSIS method for green supplier selection problem. Sustainability 2021, 13, 985. [CrossRef]

12. Krishankumar, R.; Gowtham, Y.; Ahmed, I.; Ravichandran, K.S.; Kar, S. Solving green supplier selection problem using $q$-rung orthopair fuzzy-based decision framework with unknown weight information. Appl. Soft. Comput. 2020, 94, 106431. [CrossRef]

13. Li, L.; Wu, J.; Wei, G.W.; Wang, J.; Wei, Y. Entropy-based GLDS method for social capital selection of a PPP project with $q$-rung orthopair fuzzy information. Entropy 2020, 22, 414. [CrossRef] [PubMed]

14. Gong, J.W.; Li, Q.; Yin, L.S.; Liu, H.C. Undergraduate teaching audit and evaluation using an extended MABAC method under q-rung orthopair fuzzy environment. Int. J. Intell. Syst. 2020, 35, 1912-1933. [CrossRef]

15. Tang, G.L.; Chiclana, F.; Liu, P.D. A decision-theoretic rough set model with $q$-rung orthopair fuzzy information and its application in stock investment evaluation. Appl. Soft. Comput. 2020, 91, 106212. [CrossRef]

16. Mahmood, T.; Ali, Z. A novel approach of complex $q$-rung orthopair fuzzy hamacher aggregation operators and their application for cleaner production assessment in gold mines. J. Ambient Intell. Humaniz. Comput. 2021, 12, 8933-8959. [CrossRef]

17. Jin, C.X.; Ran, Y.; Zhang, G.B. Interval-valued q-rung orthopair fuzzy FMEA application to improve risk evaluation process of tool changing manipulator. Appl. Soft. Comput. 2021, 104, 107192. [CrossRef]

18. Sun, C.; Sun, J.F.; Alrasheedi, M.; Saeidi, P.; Mishra, A.R.; Rani, P. A New Extended VIKOR Approach Using $q$-Rung Orthopair Fuzzy Sets for Sustainable Enterprise Risk Management Assessment in Manufacturing Small and Medium-Sized Enterprises. Int. J. Fuzzy Syst. 2021, 23, 1347-1369.

19. Cuong, B.C. Picture fuzzy sets. J. Comput. Sci. Cyb. 2014, 30, 409-420.

20. Tian, C.; Peng, J.J.; Zhang, S.; Zhang, W.Y.; Wang, J.Q. Weighted picture fuzzy aggregation operators and their applications to multi-criteria decision-making problems. Comput. Ind. Eng. 2019, 137, 106037. [CrossRef]

21. Ashraf, S.; Abdullah, S. Spherical aggregation operators and their application in multiattribute group decision-making. Int. J. Intell. Syst. 2019, 34, 493-523. [CrossRef]

22. Mahmood, T.; Ullah, K.; Khan, Q.; Jan, N. An approach toward decision-making and medical diagnosis problems using the concept of spherical fuzzy sets. Neural. Comput. Appl. 2019, 31, 7041-7053. [CrossRef]

23. Zedam, L.; Jan, N.; Rak, E.; Mahmood, T.; Ullah, K. An approach towards decision-making and shortest path problems based on T-spherical fuzzy information. Int. J. Fuzzy Syst. 2020, 22, 1521-1534. [CrossRef]

24. Ullah, K.; Garg, H.; Mahmood, T.; Jan, N.; Ali, Z. T-spherical fuzzy graphs: Operations and applications in various selection processes. Arab. J. Sci. Eng. 2020, 45, 2177-2193.

25. Ullah, K.; Garg, H.; Mahmood, T.; Jan, N.; Ali, Z. Correlation coefficients for T-spherical fuzzy sets and their applications in clustering and multi-attribute decision making. Soft Comput. 2020, 24, 1647-1659. [CrossRef]

26. Guleria, A.; Bajaj, R.K. On some new statistical correlation measures for T-spherical fuzzy sets and applications in soft computing. J. Inf. Sci. Eng. 2021, 37, 323-336.

27. Wu, M.Q.; Chen, T.Y.; Fan, J.P. Divergence measure of T-spherical fuzzy sets and its applications in pattern recognition. IEEE Access 2020, 8, 10208-10221. [CrossRef]

28. Ullah, K.; Mahmood, T.; Jan, N. Similarity measures for T-spherical fuzzy sets with applications in pattern recognition. Symmetry 2018, 10, 193. [CrossRef]

29. Wu, M.Q.; Chen, T.Y.; Fan, J.P. Similarity measures of T-spherical fuzzy sets based on the cosine function and their applications in pattern recognition. IEEE Access 2020, 8, 98181-98192. [CrossRef]

30. Liu, P.D.; Zhu, B.Y.; Wang, P. A Multi-attribute Decision-Making Approach Based on Spherical Fuzzy Sets for Yunnan Baiyao's R\&D Project Selection Problem. Int. J. Fuzzy Syst. 2019, 21, 2168-2191.

31. Ullah, K.; Mahmood, T.; Garg, H. Evaluation of the Performance of Search and Rescue Robots Using T-spherical Fuzzy Hamacher Aggregation Operators. Int. J. Fuzzy Syst. 2020, 22, 570-582. [CrossRef]

32. Ullah, K.; Hassan, N.; Mahmood, T.; Jan, N.; Hassan, M. Evaluation of Investment Policy Based on Multi-Attribute DecisionMaking Using Interval Valued T-Spherical Fuzzy Aggregation Operators. Symmetry 2019, 11, 357. [CrossRef]

33. Zeng, S.Z.; Garg, H.; Munir, M.; Mahmood, T.; Hussain, A. A Multi-Attribute Decision Making Process with Immediate Probabilistic Interactive Averaging Aggregation Operators of T-Spherical Fuzzy Sets and Its Application in the Selection of Solar Cells. Energies 2019, 12, 4436. [CrossRef]

34. Garg, H.; Munir, M.; Ullah, K.; Mahmood, T.; Jan, N. Algorithm for T-spherical fuzzy multi-attribute decision making based on improved interactive aggregation operators. Symmetry 2018, 10, 670. [CrossRef]

35. Liu, P.D.; Khan, Q.; Mahmood, T.; Hassan, N. T-spherical fuzzy power Muirhead mean operator based on novel operational laws and their application in multi-attribute group decision making. IEEE Access 2019, 7, 22613-22632. [CrossRef] 
36. Munir, M.; Kalsoom, H.; Ullah, K.; Mahmood, T.; Chu, Y.M. T-spherical fuzzy Einstein hybrid aggregation operators and their applications in multi-attribute decision making problems. Symmetry 2020, 12, 365. [CrossRef]

37. Ju, Y.B.; Liang, Y.Y.; Luo, C.; Dong, P.W.; Gonzale, E.D.R.S.; Wang, A.H. T-spherical fuzzy TODIM method for multi-criteria group decision-making problem with incomplete weight information. Soft Comput. 2021, 25, 2981-3001. [CrossRef]

38. Grag, H.; Ullah, K.; Mahmood, T.; Hassan, N.; Jan, N. T-spherical fuzzy power aggregation operators and their applications in multi-attribute decision making. J. Ambient Intell. Human. Comput. 2021, 12, 9067-9080, in press. [CrossRef]

39. Munir, M.; Mahmood, T.; Hussain, A. Algorithm for T-spherical fuzzy MADM based on associated immediate probability interactive geometric aggregation operators. Artif. Intell. Rev. 2021, 54, 6033-6061, in press. [CrossRef]

40. Liu, P.D.; Wang, D.Y.; Zhang, H.; Yan, L.; Li, Y.; Rong, L.L. Multi-attribute decision-making method based on normal T-spherical fuzzy aggregation operator. J. Intell. Fuzzy Syst. 2021, 40, 9543-9565. [CrossRef]

41. Mahmood, T.; Warraich, M.S.; Ali, Z.; Pamucar, D. Generalized MULTIMOORA method and Dombi prioritized weighted aggregation operators based on T-spherical fuzzy sets and their applications. Int. J. Intell. Syst. 2021, 36, 4659-4692. [CrossRef]

42. Yager, R.R. The power average operator. IEEE Trans. Syst. Man Cybern. Paart A-Syst. Hum. 2001, 31, 724-731. [CrossRef]

43. Liu, P.D.; Li, D. Some Muirhead mean operators for intuitionistic fuzzy numbers and their applications to group decision making. PLOS ONE 2017, 12, e0168767. [CrossRef]

44. Ju, Y.B.; Liu, X.Y.; Ju, D.W. Some new intuitionistic linguistic aggregation operators based on Maclaurin symmetric mean and their applications to multiple attribute group decision making. Soft Comput. 2016, 20, 4521-4548. [CrossRef]

45. Zhang, S.P.; Sun, P.; Mi, J.S.; Feng, T. Belief function of Pythagorean fuzzy rough approximation space and its applications. Int. J. Approx. Reasoning 2020, 119, 58-80. [CrossRef]

46. Zhang, X.H.; Zhou, B.; Li, P. A general frame for intuitionistic fuzzy rough sets. Inf. Sci. 2012, 216, 24. [CrossRef]

47. Liu, P.D.; Gao, H.; Fujita, H. The new extension of the MULTIMOORA method for sustainable supplier selection with intuitionistic linguistic rough numbers. Appl. Soft. Comput. 2021, 99, 106893. [CrossRef]

48. Cornelis, C.; Cock, M.D.; Kerre, E.E. Intuitionistic fuzzy rough sets: At the crossroads of imperfect knowledge. Expert Syst. 2003, 20, 260-270. [CrossRef]

49. Zhou, L.; Wu, W.Z. On generalized intuitionistic fuzzy rough approximation operators. Inf. Sci. 2008, 178, 2448-2465. [CrossRef]

50. Chinram, R.; Hussain, A.; Mahmood, T.; Ali, M.I. EDAS method for multi-criteria group decision making based on intuitionistic fuzzy rough aggregation operators. IEEE Access. 2021, 9, 10199-10216. [CrossRef]

51. Jia, F.; Liu, Y.Y.; Wang, X.Y. An extended MABAC method for multi-criteria group decision making based on intuitionistic fuzzy rough numbers. Expert Syst. Appl. 2019, 127, 241-255. [CrossRef]

52. He, Y.D.; Chen, H.Y.; Zhou, L.G.; Liu, J.P.; Tao, Z.F. Intuitionisitc fuzzy geometric interaction averaging operators and their application to multi-criteria decision making. Inf. Sci. 2014, 259, 142-159. [CrossRef]

53. Yager, R.R. On generalized Bonferroni mean operators for multi-criteria aggregation. Int. J. Approx. Reasoning 2009, 50, 1279-1286. [CrossRef]

54. Beliakov, G.; Pradera, A.; Calvo, T. Averaging Functions. In Aggregation Functions: A Guide for Practitioners; Springer: Berlin, Germany, 2008; pp. 62-67.

55. Guan, K.; Zhu, H. The generalized Heronian mean and its inequalities. Univ. Beograd. Publ. Elektrotehn. Fak-Ser. Mat. 2006, 17, 60-75. [CrossRef]

56. Liu, P.D.; You, X.L. Interval Neutrosophic Muirhead mean operators and their application in multiple attribute group decision making. Int. J. Uncertain. Quan. 2017, 7, 303-334. [CrossRef]

57. Liu, P.D. Multiple attribute group decision making method based on interval-valued intuitionistic fuzzy power Heronian aggregation operators. Comput. Ind. Eng. 2017, 108, 199-212. [CrossRef]

58. Ju, D.W.; Ju, Y.B.; Wang, A.H. Multi-attribute group decision making based on power generalized Heronian mean operator under hesitant fuzzy linguistic environment. Soft. Comput. 2019, 23, 3823-3842. [CrossRef]

59. Yu, D.J. Intuitionistic fuzzy geometric Heronian mean aggregation operators. Appl. Soft. Comput. 2013, 13, 1235-1246. [CrossRef]

60. Liu, Z.M.; Wang, S.; Liu, P.D. Multiple attribute group decision making based on $q$-rung orthopair fuzzy Heronian mean operators. Int. J. Intell. Syst. 2018, 33, 2341-2363. [CrossRef]

61. He, Y.D.; He, Z. Extensions of Atanassov's intuitionistic fuzzy interaction Bonferroni means and their application to multipleattribute decision making. IEEE Trans. Fuzzy Syst. 2016, 24, 558-573. [CrossRef]

62. Ju, Y.B.; Ju, D.W.; Gonzalez, E.D.R.S.; Giannakis, M.; Wang, A.H. Study of site selection of electric vehicle charging station based on extended GRP method under picture fuzzy environment. Comput. Ind. Eng. 2019, 135, 1271-1285. [CrossRef]

63. Xing, Y.P.; Zhang, R.T.; Wang, J.; Bai, K.Y.; Xue, J. A new multi-criteria group decision-making approach based on $q$-rung othopair fuzzy interaction Hamy mean operators. Neural Comput. Appl. 2020, 32, 7465-7488. [CrossRef]

64. Yang, Z.L.; Ouyang, T.X.; Fu, X.L.; Peng, X.D. A decision-making algorithm for online shopping using deep-learning-based opinion pairs mining and $q$-rung orthopair fuzzy interaction Heronian mean operators. Int. J. Intell. Syst. 2020, 35, 783-825. [CrossRef]

65. Wang, L.; Li, N. Pythagorean fuzzy interaction power Bonferroni mean aggreagation operators in multiple attribute decision making. Int. J. Intell. Syst. 2020, 35, 150-183. [CrossRef]

66. Onan, A. A fuzzy-rough nearest neighbor classifier combined with consistency-based subset evaluation and instance selection for automated diagnosis of breast cancer. Expert. Syst. Appl. 2015, 42, 6844-6852. [CrossRef] 
67. Wang, C.Y.; Hu, B.Q. Granular variable precision fuzzy rough sets with general fuzzy relations. Fuzzy Sets Syst. 2015, 275, 39-57. [CrossRef]

68. Ye, J. A multicriteria decision-making method using aggregation operators for simplified Neutrosophic sets. J. Intell. Fuzzy Syst. 2014, 26, 2459-2466. [CrossRef]

69. Maji, P.K.; Roy, A.R.; Biswas, R. An application of soft sets in a decision making problem. Comput. Math. Appl. 2002, 44, 1077-1083 [CrossRef]

70. Xia, M.M.; Xu, Z.S.; Chen, N. Some hesitant fuzzy aggregation operators with their application in group decision making. Group Decis. Negot. 2013, 22, 259-279. [CrossRef] 